\title{
Número mínimo de soluções para equação de Yamabe subcrítica modelada em um fibrado riemaniano com fibras mínimas
}

TESE APRESENTADA

AO,

INSTITUTO DE MATEMÁTICA E ESTATÍSTICA

DA

UNIVERSIDADE DE SÃO PAULO

PARA

OBTENÇÃA DO TÍTULO

$\mathrm{DE}$

DOUTOR EM CIÊNCIAS

Programa: Doutorado em Matemática

Orientador: Prof. Dr. Paolo Piccione

Durante o desenvolvimento deste trabalho o autor recebeu auxílio financeiro do IFCE

Data da defesa: 20 de Dezembro de 2019 


\section{Número mínimo de soluções para equação de Yamabe subcrítica modelada em um fibrado riemaniano com fibras mínimas}

Esta versão da tese contém as correções e alterações sugeridas pela Comissão Julgadora durante a defesa da versão original do trabalho, realizada em 20/12/2019. Uma cópia da versão original está disponível no Instituto de Matemática e Estatística da Universidade de São Paulo.

Comissão Julgadora:

- Prof. Dr. Paolo Piccione (Orientador) - IME-USP

- Prof. Dr. Gaetano Siciliano - IME-USP

- Prof. Dr. Renato Ghini Bettiol - BSBC-CUNY

- Prof. Dr. Ana Claudia da Silva Moreira - UFABC

- Prof. Dr. Francesco Mercuri - UNICAMP 


\section{Agradecimentos}

Ao Deus formador dos Céus e da terra, agradeço por todo bem que tem feito, em tudo vi a Sua mão, a Ele rendo graças por abrir os caminhos, direcionar meus passos, preparar o pão e confortar meu coração durante o curso, toda glória a Deus pertence.

Agradeço a Alexandra Fernandes, minha amada esposa, pelo amor, apoio e coragem durante todo o curso, juntos conseguimos transpor os obstáculos e ao nosso amado filho João Lucas pela sua alegria inspiradora, o qual sempre nos motivou.

Agradeço a minha mãe Francisca Nunes e aos meus cinco irmãos, Antonio Luis, Antonio Isidório, Neide, Lousimar e Rita, pelos ensinos, princípios e caráter que fizeram de mim uma pessoa capaz de enfrentar a vida de forma realista com fé e esperança.

Agredeço também as pessoas que de forma especial marcaram minha vida nesse período em São Paulo, Nykollas Fernandes, um irmão, meu amigo e companheiro de todas as horas, a Josilene Silva, José Aurimar, Graça Fernandes, Leonardo Fernandes, Mateus Evangelista, Michele Silva, José Maria, Gecilene Fernandes, Virgílio Fernandes, Joyce Fernandes, Jean Fernandes, Flávio e aos irmãos em Cristo Jesus da Congregação Las Palmas.

Agradeço aos amigos e colegas que sempre estiveram na torcida, prontos a estender a mão e dá uma palavra de conforto e esperança em momentos difíceis, a saber, Antonio Marcos,Felipe Felix, Marcos Rafael, Daniel Reis, Kaique Matias, Jailson Calado, Yuri Maluf, Jadevilson Cruz,Victor Hugo, Elivaldo Macedo, Rodrigo Santos, Eurípedes Silva, Benigno, Pablo, Edson Sampaio, José Eduardo, Leo Ivo, Rui Eduardo, Oscar, Michael Rodrigues, Francisco José, Arquimedes Albuquerque, Irailma Melo, Roberta da Silva, Pedro Saraiva.

Agradeço ao meu orientador, o Professor Paolo Piccione, pelo direcionamento durante o curso e aos professores Alexandre Fernandes, Mário de Assis, Francisco Regis, Wilson, Gláucio Terra, Gaetano Siciliano e Marcos Alexandrino pelo apoio. Agradeço a disponibilidade da comissão julgadora, a secretaria da Pós-Graduação em Matemática do IME-USP, em particular a Ana Paula, Maju e Henrique Guetti, sempre prontos a ajudar.

Agradeço Instituto Federal de Educação, Ciência e Tecnologia do Ceará - IFCE pelo apoio financeiro. 


\section{Resumo}

Neto, J. N. A. Número mínimo de soluções para equação de Yamabe subcrítica modelada em um fibrado riemaniano com fibras mínimas. 2019. 65 f. Tese (Doutorado) Instituto de Matemática e Estatística, Universidade de São Paulo, São Paulo, 2019.

Neste trabalho, modelamos o Problema de Yamabe em um fibrado riemanianno determinado por uma submersão riemanianna com fibras mínimas. Este modelo consiste de uma equação elíptica subcrítica com os coeficientes adequados ao contexto acima, inspirados pela técnica desenvolvida por Vieri Benci, Claudio Bonanno e Anna Maria Micheletti no artigo On the multiplicity of solutions of a nonlinear elliptic problem on riemannian manifolds. Definimos funções usando o conceito de centro de massa riemanianno, de modo que as condições fossem satisfeitas para aplicação da teoria de Lusternik- Schnirelmann garantindo a existência de um número mínimo de soluções para o Problema de Yamabe em um fibrado riemanianno com fibras mínimas.

Palavras-chave: Equações Elípticas, Submersão, multiplicidade. 


\section{Abstract}

Neto, J. N. A. Número mínimo de soluções para equação de Yamabe subcrítica modelada em um fibrado riemaniano com fibras mínimas. 2019. 65 f. Tese (Doutorado) Instituto de Matemática e Estatística, Universidade de São Paulo, São Paulo, 2019.

In this paper we model the Yamabe Problem in a Riemannian fiber bundle determined by a Riemannian submersion with minimal fibers. This model consists of a subcritical elliptic equation with adequate coefficients to the context above, inspired by the technique developed by Vieri Benci, Claudio Bonanno and Anna Maria Micheletti in the article "On the multiplicity of solutions of a nonlinear elliptic problem on riemannian manifolds". Are define functions using the concept of the riemannian center of mass, so that the conditions are satisfied for the application of LusternikSchnirelmann's theory, ensuring a minimum number of solutions to the Yamabe Problem in such a riemannian fiber bundle.

Keywords: Elliptic equations, Submersion, multiplicity. 


\section{Sumário}

$\begin{array}{ll}\text { Introdução } & 1\end{array}$

\begin{tabular}{lll}
\hline & Geometria Riemanniana & 3
\end{tabular}

1.1 Variedades Diferenciáveis, Espaço Tangente, Aplicações Diferenciáveis . . . . . . . 3

1.2 Variedades Riemannianas $\ldots \ldots \ldots \ldots \ldots \ldots \ldots$

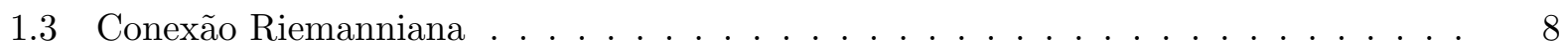

1.4 Geodésica, Vizinhança Normal, Curvaturas $\ldots \ldots \ldots$. . . . . . . . . . . . . 10

1.5 Aplicação Exponencial $\ldots \ldots \ldots \ldots$. . . . . . . . . . . . . . . . . . . 11

1.6 Curvaturas . . . . . . . . . . . . . . . . . . . . . . . . . . . . . . . . 11

1.7 Gradiente, Divergente e Laplaciano $\ldots \ldots \ldots$. . . . . . . . . . . . . . . . . . 12

1.8 Geometria Conforme . . . . . . . . . . . . . . . . . . . . . . . . . . . . . . . 14

1.8 .1 Curvatura Riemanniana Conforme . . . . . . . . . . . . . . . . . 15

1.8 .2 Curvatura de Ricci Conforme . . . . . . . . . . . . . . . . 16

1.8 .3 Curvatura Escalar Conforme . . . . . . . . . . . . . . . . . . . 18

\begin{tabular}{|lll}
2 & Os Espaços de Sobolev & 19
\end{tabular}

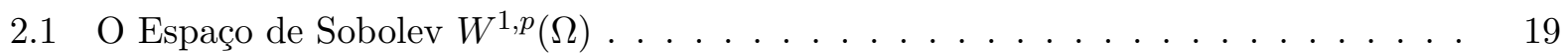

2.2 O Espaço de Sobolev $W^{1, p}(M)$

2.3 As imersões de Sobolev sobre a Variedade $M$. . . . . . . . . . . . . . . . . 23

2.4 Um problema Elíptico não linear em variedade riemanniana . . . . . . . . . . . . . 24

\begin{tabular}{|lll}
3 & Problema de Yamabe - subcrítico & 27
\end{tabular}

$3.1 \quad$ Problema de Yamabe . . . . . . . . . . . . . . . . . . . . . . . 27

3.2 Problema de Yamabe Subcrítico $\ldots \ldots \ldots \ldots \ldots \ldots \ldots$

4 Número mínimo de soluções para Problema de Yamabe-subcrítico 37

4.1 Centro de Massa Riemaniana $\ldots \ldots \ldots \ldots \ldots \ldots$. . . . . . . . . . . . . . 37

$4.1 .1 \quad$ A Função Centro de Massa $c_{m}(r, \eta): L_{r, \eta}^{1}(M) \rightarrow M \ldots \ldots . \ldots . . \ldots 40$

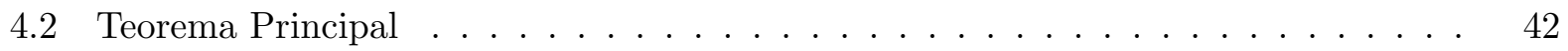

$4.2 .1 \quad$ Construção de uma função radial $\phi_{\varepsilon} \ldots \ldots \ldots \ldots$. . . . . . . . . 44

4.2 .2 Demonstração do Teorema Principal . . . . . . . . . . . . . . . . . . . . 52 


\section{Introdução}

A geometria riemaniana surge com a necessidade de generalizar a teoria geométrica desenvolvida para superfícies.

No estudo de superfícies, um resultado clássico de uniformização garante que para uma superfície $(M, g)$, existe uma função suave $u>0$ em $M$ e uma métrica $\tilde{g}=u^{2} g$ tal que $(M, \tilde{g})$ possui curvatura gaussiana constante. Dizemos que $\tilde{g}$ é uma métrica conforme (multiplicação de $g$ por uma função positiva). Encontrar métricas conformes é um tema que tem motivado o desenvolvimento da análise geométrica desde o século passado.

D. Hilbert em 1915 [12] prova que uma métrica $g$ sobre uma superfície compacta $M$ é Einstein se e somente se $g$ é ponto crítico do funcional $Y: M \rightarrow \mathbb{R}$ dado por

$$
Y(g)=\frac{\int_{M} S_{g} d v_{g}}{\operatorname{Vol}_{g^{\frac{m-2}{m}}}(M)},
$$

onde $S_{g}$ é a curvatura escalar da métrica $g, v_{g}$ denota a forma de volume induzida por $g, m$ é a

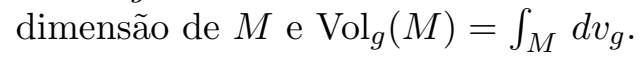

Quando $Y$ é restrito a classe conforme $[g]$ da métrica $g$, decorre que os pontos críticos de $Y_{g}$ nestas configurações são as métricas conformes a $g$ com curvatura escalar constante.

Em 1960, H. Yamabe [21] formula a seguinte conjectura: "toda variedade Riemaniana compacta $(M, g)$ de dimensão $m \geq 3$, admite uma métrica conforme $g$ com curvatura escalar constante". Essa conjectura ficou conhecida como Problema de Yamabe, o qual foi completamente resolvido em 1984, com as contribuições de H. Yamabe [21], S. Trudinger [20], T. Aubin [2] e R. Schoen [18].

Consideremos uma variedade riemaniana fechada de dimensão $m \geq 3$. A métrica $\tilde{g}=f^{p_{k}-2} \cdot g$ conforme a $g$ tem curvatura escalar constante $\mu \in \mathbb{R}$ se e somente se a função positiva $f$ satisfaz a Equação de Yamabe correspondente a $g$ :

$$
-a_{m} \Delta_{g} f+S_{g} f=\mu f^{p_{m}-1}
$$

onde $a_{m}=\frac{4(m-1)}{m-2}, p_{m}=\frac{2 m}{m-2}$ é o expoente crítico de Sobolev e $S_{g}$ denota a curvatura escalar de $g$.

Considere o funcional Hilbert-Eistein $Y_{g}$ restrito a classe conforme de $g$ :

$$
\begin{array}{r}
\tilde{g} \in[g] \Rightarrow \tilde{g}=f^{p_{m}-2} \cdot g \\
Y(\tilde{g})=Y\left(f^{p_{m}-2} \cdot g\right)=Y_{g}(f)=\frac{E_{g}(f)}{\|f\|_{p_{m}}^{2}}
\end{array}
$$

onde $E_{g}(f)=\int_{M}\left(a_{m}\|\nabla f\|^{2}+S_{g} f^{2}\right) d v_{g}$ e $\|f\|_{p_{m}}=\left(\int_{M} f^{p_{m}}\right)^{\frac{1}{p_{m}}}$. As soluções da equação de Yamabe são os pontos críticos do funcional Hilbert-Einstein $Y$ restrito a $[g]$ e $d v_{g}$ é a forma de volume associada a $g$. 
O funcional $Y_{g}$ é limitado inferiormente para qualquer métrica $g$. Além disso o ínfimo é atingido. $\inf \left(Y_{g}\right)$ é chamado constante de Yamabe e denotado por $Y(M,[g])$.

$$
Y(M,[g])=\inf _{f \in C_{+}^{\infty}(M)}\left(Y_{g}(f)\right),
$$

onde $C_{+}^{\infty}(M)$ é o conjunto das funções suave positivas de $M$.

Quando $Y(M,[g]) \leq 0$ a equação de Yamabe tem uma única solução, a menos de homotetias. Contudo, no caso positivo existem soluções múltiplas, como podemos ver em Obata [15], Schoen [18] e Pollack [17, neste artigo Daniel Pollack mostra que qualquer classe conforme com constante de Yamabe positiva pode ser aproximada na $C^{0}$-topologia por uma classe conforme com um número arbitrariamente grande de métricas, não isométricas, com curvatura escalar constante.

Em 2012 (De Lima,P.Piccione, Zedda) em [8] usando Teoria de Bifurcações obtiveram resultados de multiplicidade de soluções para problema de Yamabe em produto Riemaniano, eles consideraram duas variedades riemanianas compactas sem bordo $\left(M_{0}, g_{0}\right)$ e $\left(M_{1}, g_{1}\right)$ definem $M=\left(M_{0} \times M_{1}, g_{\lambda}\right)$, onde $g_{\lambda}=g_{0} \oplus \lambda g_{1}, \lambda \in(0,+\infty)$ é uma família de métricas, provam que existe um conjunto enumerável $\Lambda \subseteq(0,+\infty)$ que se acumula somente em 0 e $+\infty$, tal que:

1. A família $\left(g_{\lambda}\right)$ é localmente rígida em todo ponto de $(0,+\infty) \backslash \Lambda$, isto é, $\forall \bar{\lambda} \in(0,+\infty) \backslash \Lambda$, qualquer métrica de curvatura escalar constante sobre $M$ suficientemente $C^{2, \alpha}$-próxima à $g_{\bar{\lambda}}$ é homotética à algum elemento da forma $g_{\bar{\lambda}}$.

2. Em cada $\lambda_{*} \in \Lambda$, a menos de um subconjunto finito, existe um ramo de bifurcação de métricas que não pertencem à família $\left(g_{\lambda}\right)$.

Também obtiveram resultados de rigidez e bifurcação quando as curvaturas escalares de $g_{0}$ e $g_{1}$ não são ambas positivas.

R. Bettiol e P Piccione também contribuiram muito para o desenvolvimento dessa linha de pesquisa com diversos resultado publicados, veja [5], [6], [7] motivando o trabalho de J. Petean obteve um resultado de multiplicidade em produtos riemanianos usando a teoria de LusternikSchnirelmann, ele considerou $\left(M^{m}, g\right)$ e $\left(N^{n}, h\right)$ variedades riemanianas fechadas, de tal forma que $\left(W^{k} \cdot g_{\delta}\right)=(M \times N, g+\delta h)$ onde $k=m+n$ e $\delta \in \mathbb{R}_{>0}$. Nestas condições,buscou encontrar soluções da equação de Yamabe correspondentes a métrica $g_{\delta}$ no caso é subcritica $\left(p_{m+n}<p_{n}\right)$, além disso ele considerou soluções que dependem de apenas de um dos fatores.

Nesse contexto iniciamos nossa pesquisa, percebemos que seria possível usar o método da variedade de Nehari juntamento com a teoria de Lusternik-Schnirelmann para obter um resultado análogo ao produzido por J. Petean [16], onde ele aplicou o a técnica de Vieri Benci, Claudio Bonanno, e Anna Maria Micheletti [3]. Em síntese nossa pesquisa preenche uma lacuna no que se refere ao Problema de Yamabe, pois obtivemos um resultado de multiplicidade para a equação de Yamabe modela em um fibrado riemanianno com fibras mínimas, tal fibrado é obtido via uma submersão riemanianna, mostramos a existência de outras soluções proximas de uma sulução de menor energia. Este trabalho está dividido em quatro capítulos, no capítulos 1 fizemos uma compilação dos resultados preliminares de geometria riemaniana, no capítulo 2 falamos sobre alguns conceitos de espaços de Sobolev, no capítulo 3 falamos sobre o caso subcrítico do problemas de Yamabe e finalmente no capítulo 4 falamos sobre o problema de Yamabe no contexto de fibrados riemaniana e provamos o seguinte resultado de multiplicidade: Seja $\left(M^{m}, g_{M}\right)$ uma variedade riemanniana compacta, com $m \geq 3$ e $\left(B^{n}, g_{B}\right)$ uma variedade riemanniana. Se $\pi:\left(M^{m}, g_{M}\right) \rightarrow\left(B^{n}, g_{B}\right)$ é uma submersão riemanniana com fibras mínimas e de curvatura escalar constante positiva, então, para $\varepsilon>0$ suficientemente pequeno, existem Cat $(B)+1$ métricas conformes à $g_{M}^{\varepsilon}=g_{B}+\varepsilon g_{\mathcal{F}}$ com curvatura escalar constante. Onde Cat é a categoria de Lusternik-shnirelmann. 


\section{Capítulo 1}

\section{Geometria Riemanniana}

Neste capítulo apresentamos alguns conceitos importantes em geometria riemanniana, definições e propriedades que usaremos no decorrer do texto, não temos o objetivo de demonstrar os resultados clássicos dessa teoria a menos que os mesmos venham a contribuir para a clareza do trabalho de um modo geral. Os resultados seguintes foram baseados em [9] e [14]

\subsection{Variedades Diferenciáveis, Espaço Tangente, Aplicações Dife- renciáveis}

Definição 1.1 Uma variedade diferenciável $M$, de dimensão $m$, é um espaço topológico Hausdorff, com base enumerável e uma estrutura diferenciáveis, isto é, uma coleção de cartas $\mathcal{A}=\left\{\left(U_{\alpha}, \phi_{\alpha}\right)\right\}$, chamada atlas, onde a aplicação $\phi_{\alpha}: U_{\alpha} \rightarrow V_{\alpha}$, é um homeomorfismo, $U_{\alpha} \subset \mathbb{R}^{m}$, para todo $\alpha$, tal que

1. $M=\cup_{\alpha} V_{\alpha}=\cup_{\alpha} \phi_{\alpha}\left(U_{\alpha}\right)$,

2. para cada par de cartas $\left(U_{\alpha}, \phi_{\alpha}\right),\left(U_{\beta}, \phi \beta\right)$ onde $\phi_{\alpha}\left(U_{\alpha}\right) \cap \phi_{\beta}\left(U_{\beta}\right)=V_{\alpha} \cap V_{\beta} \neq \emptyset$, as aplicações $\phi_{\beta}^{-1} \circ \phi_{\alpha}: \phi_{\alpha}^{-1}\left(V_{\alpha} \cap V_{\beta}\right) \rightarrow \phi_{\beta}^{-1}\left(V_{\alpha} \cap V_{\beta}\right)$ são diferenciáveis.

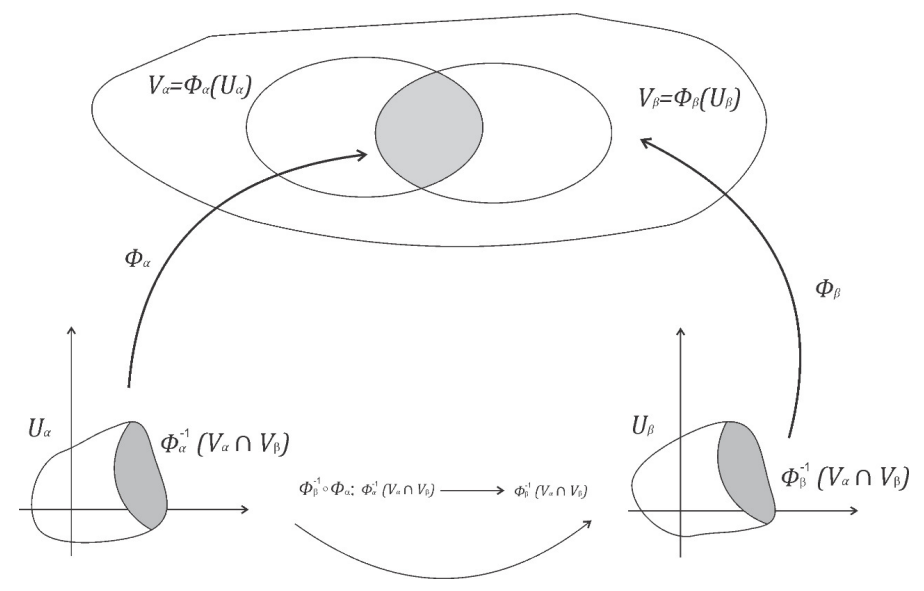

Usaremos a notação $M^{m}$ para denotar um objeto que satisfaz a definição 1.1 , o par $\left(U_{\alpha}, \phi_{\alpha}\right) \in \mathcal{A}$ com $p \in \phi_{\alpha}\left(U_{\alpha}\right)$ é chamado parametrização ou sistema de coordenadas de $M^{m}$ em $p$ e $\phi_{\alpha}\left(U_{\alpha}\right)$ chamada vizinhança coordenada de $p$. 
Exemplos 1.2 Os seguintes conjuntos são variedades diferenciáveis: $\mathrm{O}$ espaço euclidiano $\mathbb{R}^{n}$, o espaço projetivo $P^{n}(\mathbb{R})$ e a esfera $S^{n-1}=\left\{x \in \mathbb{R}^{n} ;|x|^{2}=\sum_{i=1}^{n} x^{2}=1\right\}$

Definição $1.3 \mathrm{O}$ subconjunto $S \subset M^{m}$ é uma subvariedade, de dimensão $n$, da variedade $M^{m}$, se para todo $p \in S$, existe uma carta $\left(U_{\alpha}, \phi_{\alpha}\right)$ de $M^{m}$, com $p \in U_{\alpha}$, tal que $\phi_{\alpha}\left(U_{\alpha} \cap S\right)=$ $\phi_{\alpha}\left(U_{\alpha}\right) \cap \mathbb{R}^{n} \times\{0\}$.

A definição 1.1 permite estender o conceito de aplicações difenciáveis em variedades, como aparece a seguinte definição.

Definição 1.4 Sejam $M^{m}$ e $N^{n}$ variedades diferenciáveis. Uma aplicação $f: M^{m} \rightarrow N^{n}$ é diferenciável em $p \in M^{m}$ se dada uma parametrização $\phi_{\beta}: U_{\beta} \subset \mathbb{R}^{n} \rightarrow N^{n}$ em $f(p)$ existe uma parametrização $\phi_{\alpha}: U_{\alpha} \subset \mathbb{R}^{m} \rightarrow M^{n}$ em $p$ tal que $f\left(\phi_{\alpha}\left(U_{\alpha}\right)\right) \subset \phi_{\beta}\left(U_{\beta}\right)$ e a aplicação é:

$$
\phi_{\beta}^{-1} \circ f \circ \phi_{\alpha}: U_{\alpha} \subset \mathbb{R}^{m} \rightarrow \mathbb{R}^{n}
$$

é diferenciável em $\phi_{\alpha}^{-1}(p)$

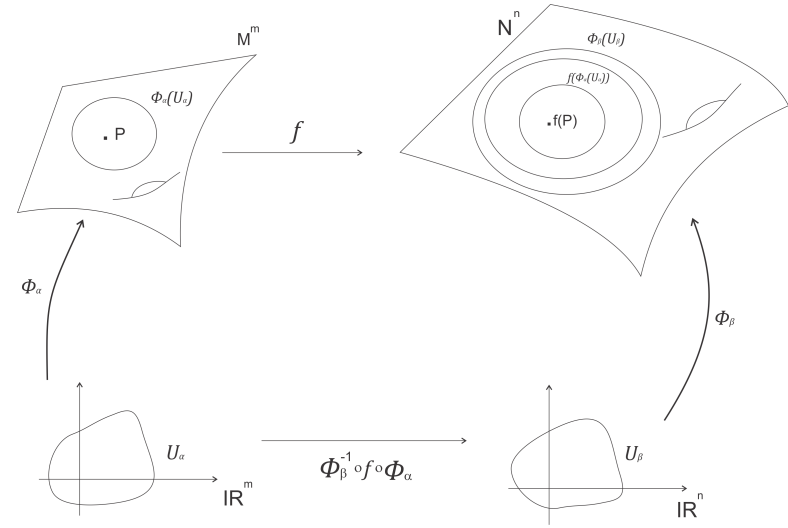

Definição 1.5 Seja $M^{m}$ um variedade diferenciável e $\gamma:(-\epsilon, \epsilon) \rightarrow M^{m}$ uma curva diferenciável em $M^{m}$. Suponha que $\gamma(0)=p \in M^{m}$, e seja $\mathcal{D}$ o conjunto das funções diferenciáveis definidas em $p$. O vetor tangente à curva $\gamma$ em $t=0$ é a função $\gamma^{\prime}(0): \mathcal{D} \rightarrow \mathbb{R}$ dada por

$$
\gamma^{\prime}(0) f=\left.\frac{d(f \circ \gamma)}{d t}\right|_{t=0}, f \in \mathcal{D}
$$

$T_{p} M$ é chamdo espaço tangente a $M$ no ponto $p \in M$ e é formado por todos os vetores tangentes a $M^{m}$ em $p$. Agora vamos considerar um sistema de coordenadas para uma melhor compreensão dos vetores tangentes em uma variedade. Seja $\phi: U \rightarrow M$ uma carta em $p=\phi(0)$ e $f \in \mathcal{D}$, em 


\subsection{VARIEDADES DIFERENCIÁVEIS, ESPAÇO TANGENTE, APLICAÇÕES DIFERENCIÁVEIS5}

termos desta parametrização

$$
\begin{aligned}
\gamma^{\prime}(0) f & =\left.\frac{d}{d t}(f \circ \gamma)\right|_{t=0} \\
& =\left.\frac{d}{d t} f\left(x_{1}(t), \ldots, x_{m}(t)\right)\right|_{t=0} \\
& =\sum_{i=1}^{m} x_{i}^{\prime}(0)\left(\frac{\partial f}{\partial x_{i}}\right)_{0} \\
& =\left(\sum_{i=1}^{m} x_{i}^{\prime}(0)\left(\frac{\partial}{\partial x_{i}}\right)_{0}\right) f
\end{aligned}
$$

donde obtemos a seguinte expressão para $\gamma^{\prime}(0)$ no sistema de coordenadas $\phi$ é

$$
\gamma^{\prime}(0)=\sum_{i=1}^{m} x_{i}^{\prime}(0)\left(\frac{\partial}{\partial x_{i}}\right)_{0}
$$

. Observe que $\left(\frac{\partial}{\partial x_{i}}\right)_{0}$ é o vetor tangente em $p$ à curva parametrizada $\gamma\left(x_{i}\right)=\phi\left(0, \ldots 0, x_{i}, 0, \ldots, 0\right)$, portanto podemos assumir que o conjunto

$$
\left\{\left(\frac{\partial}{\partial x_{1}}\right)_{0}, \ldots,\left(\frac{\partial}{\partial x_{m}}\right)_{0}\right\}
$$

gera o espaço $T_{p} M$.

Proposição 1.6 Sejam $M^{m}$ e $N^{n}$ variedades diferenciáveis e seja $f: M^{m} \rightarrow N^{n}$ uma aplicação diferenciável. Para cada ponto $p \in M^{m}$ e cada vetor $v \in T_{p} M$, escolha um curva difenciável $\gamma:(-\epsilon, \epsilon) \rightarrow M^{m} \operatorname{com} \gamma(0)=p$ e $\gamma^{\prime}(0)=v . \quad A$ aplicação $d f_{p}: T_{p} M \rightarrow T_{f(p)} N$ dada por $d f_{p}(v)=(f \circ \gamma)^{\prime}(0)$ é linear e não depende da escolha de $\gamma$.

\section{Prova:}

Veja Carmo, Manfredo P. do [9].

A aplicação definida anteriormente $d f_{p}: T_{p} M \rightarrow T_{f(p)} N$ é chamando difencial de $f$ em $p$, o qual é linear e cuja matriz associada as parametrizações $x: U \subset \mathbb{R}^{m} \rightarrow M^{n}$ e $y: V \subset \mathbb{R}^{n} \rightarrow N^{n}$ é dada por $\left(\frac{\partial y_{i}}{\partial x_{j}}\right)_{n \times m}$.

Definição 1.7 Dizemos que $M^{m}$ é orientável se possui um atlas maximal $\mathcal{A}$ orientado, isto é, o determinante da mudança de coordenadas $\phi_{\beta}^{-1} \circ \phi_{\alpha}$ é positivo para quaisquer duas cartas $\left(U_{\alpha}, \phi_{\alpha}\right),\left(U_{\beta}, \phi_{\beta}\right)$ deste atlas, em cada ponto de interseção $\phi_{\alpha}\left(U_{\alpha}\right) \cap \phi_{\beta}\left(U_{\beta}\right)$.

Definição 1.8 Uma aplicação $f: M^{m} \rightarrow N^{n}$ é chamada difeomorfismo quando ela é diferenciável, bijetora e sua inversa $f^{-1}$ é diferenciável. Quando $p \in U \subset M^{m}$ e $f(p) \in V \subset N^{n}$ se a restrição $f: U \rightarrow V$ é difeomorfismo, dizemos que $f$ é difeomorfismo local.

Definição 1.9 Uma aplicação $f: M^{m} \rightarrow N^{n}$ é chamada imersão quando $d f_{p}: T_{p} M \rightarrow T_{f(p)} N$ é injetora para todo $p \in M^{m}$ e $(m \geq n)$. Além disso, quando $f$ é um homeomorfismo sobre a imagem $f\left(M^{m}\right) \subset N^{n}$, onde $f\left(M^{m}\right)$ tem a topologia induzida por $N^{n}, f$ é chamado mergulho. 
Definição 1.10 Uma aplicação $f: M^{m} \rightarrow N^{n}$ é chamada submersão quando $d f_{p}: T_{p} M \rightarrow T_{f(p)} N$ é sobrejetor para todo $p \in M^{m}$.

\section{Fato 1.11}

- O conjunto $T M=\left\{(p, v) ; p \in M^{m}, v \in T_{p} M\right\}$ é uma variedade diferenciavel chamada fibrado tangente de $M^{m}$ e tem dimensão $2 m$, uma carta para $T M$ é da forma

$$
\psi_{\alpha}: U_{\alpha} \times \mathbb{R}^{m} \rightarrow T M
$$

onde

$$
\psi_{\alpha}\left(x_{1}^{\alpha}, \cdots, x_{m}^{\alpha}, u_{1}, \cdots, u_{m}\right)=\left(\phi_{\alpha}\left(x_{1}^{\alpha}, \cdots, x_{m}^{\alpha}\right), \sum_{i=1}^{m} u_{i} \frac{\partial}{\partial x_{i}^{\alpha}}\right)
$$

para uma carta $\left(U_{\alpha}, \phi_{\alpha}\right)$ que suas coordenadas são indicadas por $\left(x_{1}^{\alpha}, \cdots, x_{m}^{\alpha}\right)$, o conjunto $\left\{\frac{\partial}{\partial x_{1}^{\alpha}}, \cdots, \frac{\partial}{\partial x_{m}^{\alpha}}\right\}$ é uma base associada aos espaços tangentes de $\phi_{\alpha}\left(U_{\alpha}\right)$.

- Um conjunto $S \subset M^{m}$ é uma subvariedade mergulhada de $M^{m}$ quando tem a toplogia induzida por $M^{m}$ e a inclusão é um mergulho.

- Dada uma aplicação $f: M^{m} \rightarrow N^{n}$, dizemos que ponto $q \in N^{n}$ é um valor regular para $f$, se $d f_{p}$ é sobrejetora para todo $p \in f^{-1}(q)$.

Teorema 1.12 Seja $f: M^{m} \rightarrow N^{n}$ uma aplicação diferenciável, $n \leq m$ e $q \in N^{n}$ valor regular de $f$, então $S=f^{-1}(q)$ é uma subvariedade mergulhada de $M$, com dimensão $(m-n)$ e $T_{p} S=$ $\operatorname{Ker}(d f)_{p}$ para todo $p \in S$.

Definição 1.13 Um campo de vetores diferenciável $X$ em uma variedade diferenciável $M^{m}$ é uma aplicação diferenciável $X: M^{m} \rightarrow T M$ que a cada ponto $p \in M^{m}$ associa um vetor tangente $X(p) \in T_{p} M$. Para um sistema de coordenadas $\phi: U \subset \mathbb{R}^{m} \rightarrow M^{m}$ podemos escrever

$$
\begin{aligned}
X: M^{m} & \rightarrow T M \\
p & \longmapsto X(p)=\sum_{i=1}^{m} a_{i}(p) \frac{\partial}{\partial x_{i}}
\end{aligned}
$$

onde $a_{i}: U \rightarrow \mathbb{R}$ são funções diferenciáveis em $U$ e $\left\{\frac{\partial}{\partial x_{i}}\right\}_{i=1}^{m}$ é uma base associada ao sistema de coordenadas $x$. Donde

$$
(X f) p=\sum_{i=1}^{m} a_{i}(p) \frac{\partial f}{\partial x_{i}}=d f_{p}(X)
$$

para um função diferenciável $f: M^{m} \rightarrow \mathbb{R}$.

Denotamos por $\mathfrak{X}(M)$ o conjunto dos campos diferenciáveis de classe $C^{\infty}$ em $M^{m}$

Proposição 1.14 Sejam $X$ e $Y$ compos de vetores diferenciáveis em uma variedade diferenciável $M^{m}$. Então existe um único campo vetorial $Z$ tal que, $Z f=(X Y-Y X) f$, onde $f \in C^{\infty}(M)$.

\section{Prova:}

Veja página 28 do livro Geometria Riemanniana de Manfredo P. do Carmo [9]. 
Definição 1.15 O campo vetorial diferenciável $Z$ dado na proposição 1.14 é chamado colchete de Lie dos campos $X$ e $Y$, notação

$$
[X, Y]=X Y-Y X
$$

Proposição 1.16 Sejam $X, Y$ e $Z$ pertencentes a $\mathfrak{X}(M), \alpha, \beta \in \mathbb{R}$ e $f, g \in C^{\infty}(M)$, então:

1. $[X, Y]=-[Y, X]$

2. $[\alpha X+\beta Y, Z]=\alpha[X, Z]+\beta[Y, Z]$

3. $[[X, Y], Z]+[[Y, Z], X]+[[Z, X], Y]=0$

4. $[f X, g Y]=f g[X, Y]+f X(g) Y-g Y(f) X$

\section{Prova:}

A prova dos itens 1. e 2. decorre diretamente da definição 1.15. Para mostrar o item 3. basta desenvolver a primeira parcela e depois a segunda e terceira parcelas e compará-las.

$$
\begin{aligned}
{[[X, Y], Z] } & =[X Y-Y X, Z] \\
& =X Y Z-Y X Z-Z X Y+Z Y X .
\end{aligned}
$$

Agora vamos desenvolver as outras duas parcelas,

$$
\begin{aligned}
{[[Y, Z], X]+[[Z, X], Y] } & =X Y Z-X Z Y-Y Z X+Z Y X \\
& =Y Z X-Y X Z-Z X Y+X Z Y
\end{aligned}
$$

Usando o item 1. concluimos a prova. Para mostra o item 4. basta aplicarmos a definição 1.15

$$
\begin{aligned}
{[f X, g Y] } & =f X(g Y)-g Y(f X) \\
& =f g X Y+f X(g) Y-g f Y X-g Y(f) X \\
& =f g[X, Y]+f X(g) Y-g Y(f) X .
\end{aligned}
$$

\subsection{Variedades Riemannianas}

Definição 1.17 Uma métrica riemanniana $g$ em uma variedade diferenciável $M^{m}$ é uma aplicação $g: T_{p} M \times T_{p} M \rightarrow \mathbb{R}$ que associa a cada ponto $p \in M$ um produto interno $\langle,\rangle_{p}$, definido em $T_{p} M \times T_{p} M$ que varia suavemente com $p$, tal que, se $x: U \subset \mathbb{R}^{n} \rightarrow M^{n}$ é um sistema de coordenadas em $p, x\left(x_{1}, \ldots, x_{m}\right)=p \in x(U)$ e $\frac{\partial}{\partial x_{i}}(p)=d x(0, \cdots, 1, \cdots)$ então

$$
g_{i j}\left(x_{1}, \cdots, x_{m}\right)=\left\langle\frac{\partial}{\partial x_{i}}, \frac{\partial}{\partial x_{j}}\right\rangle_{p}
$$

é uma função diferenciável em $U$.

Uma variedade diferenciável $M^{m}$ munida de uma métrica riemanniana $g$ é chamada variedade riemanniana e representada como $\left(M^{m}, g\right)$ e $g_{i j}$ é a expressão local da métrica no sistema de coordenadas $x: U \subset \mathbb{R}^{m} \rightarrow M^{n}$. 
Exemplos 1.18 Métrica produto. Sejam $M^{m}$ e $N^{n}$ variedades riemannianas e considere o produto cartesiano $M^{m} \times N^{n}$ com estrutura diferenciável produto. Considere as projeções naturais

$$
\begin{gathered}
\pi_{M}: M^{m} \times N^{n} \rightarrow M^{m} \\
\pi_{N}: M^{m} \times N^{n} \rightarrow N^{n}
\end{gathered}
$$

temos uma métrica da seguinte forma

$$
\langle u, v\rangle_{(p, q)}=\left\langle d \pi_{M} \cdot u, d \pi_{M} \cdot v\right\rangle_{p}+\left\langle d \pi_{N} \cdot u, d \pi_{N} \cdot v\right\rangle_{q}
$$

para todo $(p, q) \in M^{m} \times N^{n}, u, v \in T_{(p, q)} M^{m} \times N^{n}$.

Podemos agora definir volume de uma variedade riemanniana $M^{m}$ orientada. Seja $p \in M^{m}$ e $\phi: U \subset \mathbb{R}^{m} \rightarrow M^{m}$ um sistema de coordenadas em $p \in M^{m}$ e uma base ortonormal $\left\{e_{1}, \cdots, e_{m}\right\}$ em $T_{p} M$ ambos com a mesma orientação de $M$, escrevendo $X_{i}(p)=\frac{\partial}{\partial x_{i}}(p)$ na base $\left\{e_{i}\right\}$ teremos $X_{i}(p)=\sum_{i j} a_{i j} e_{j}$. Então

$$
g_{i k}(p)=\left\langle X_{i}, X_{j}\right\rangle(p)=\sum_{j l} a_{i j} a_{k l}\left\langle e_{j}, e_{l}\right\rangle=\sum_{j} a_{i j} a_{k l}
$$

como o $\operatorname{vol}\left(X_{1}(P), \cdots, X_{m}(p)\right)=\operatorname{vol}\left(e_{1}, \cdots, e_{m}\right) \cdot \operatorname{det}\left(a_{i j}\right)=\sqrt{\operatorname{det}\left(g_{i j}\right)}(p)$.

Por conseguinte dado um sistema de coordenadas $\psi: V \subset \mathbb{R}^{m} \rightarrow M^{m}$ em $p \in M^{m}$ com a mesma orientação de $M^{m}, \operatorname{com} Y_{i}(p)=\frac{\partial}{\partial y_{i}}(p)$ e $h_{i j}(p)=\left\langle X_{i}, X_{j}\right\rangle(p)$

$$
\begin{aligned}
\sqrt{\operatorname{det}\left(g_{i j}\right)}(p) & =\operatorname{vol}\left(X_{1}(p), \cdots, X_{m}(p)\right) \\
& =J \operatorname{vol}\left(Y_{1}(p), \cdots, Y_{m}(p)\right) \\
& =J \sqrt{\operatorname{det}\left(h_{i j}\right)}(p)
\end{aligned}
$$

onde $J=\operatorname{det}\left(\frac{\partial y_{i}}{\partial x_{j}}\right)=\operatorname{det}\left(d \psi^{-1} \circ d \phi\right)(p)>0$. Para uma região $R \subset \phi(U) \subset M^{m}$ aberta, conexa com fecho compacto e cuja fronteira de $\phi^{-1}(R)$ tem medida nula em $\mathbb{R}^{m}$, temos que

$$
\operatorname{vol}(R)=\int_{\phi^{-1}(R)} \sqrt{\operatorname{det}\left(g_{i j}\right)} d x_{1} \cdots d x_{m}
$$

\subsection{Conexão Riemanniana}

Definição 1.19 Uma conexão afim $\nabla$ em uma variedade diferenciável $M^{m}$ é uma aplicação

$$
\begin{aligned}
\nabla: \mathfrak{X}(M) \times \mathfrak{X}(M) & \longrightarrow \mathfrak{X}(M) \\
(X, Y) & \longmapsto \nabla_{X} Y
\end{aligned}
$$

que satisfaz as propriedades abaixo

1. $\nabla_{f X} Y=f \nabla_{X} Y$

2. $\nabla_{f X+g Y} Z=f \nabla_{X} Z+g \nabla_{Y} Z$,

3. $\nabla_{X}(Y+Z)=\nabla_{X} Y+\nabla_{X} Z$ 
4. $\nabla_{X}(f Y)=f \nabla_{X} Y+X(f) Y$ onde $X, Y, Z \in \mathfrak{X}(M)$ e $f, g \in \mathcal{D}(M)$

Diante disto, considere um ponto $p \in M^{m}$ e um sistema de coordenadas $\phi=\left(x_{1}, \ldots, x_{m}\right)$ em torno de $p$, sejam $X, Y \in \mathfrak{X}(M)$ podemos escrever

$$
\begin{aligned}
& X=\sum_{i} x_{i} X_{i} \\
& Y=\sum_{j} y_{j} X_{j}
\end{aligned}
$$

onde $X_{i}=\frac{\partial}{\partial x_{i}}$

$$
\begin{aligned}
\nabla_{X} Y & =\sum_{i} x_{i} \nabla_{X i}\left(\sum_{j} y_{j} X_{j}\right) \\
& =\sum_{i j} x_{i} y_{j} \nabla_{X_{i}} X_{j}+\sum_{i j} x_{i} X_{i}\left(y_{j}\right) X_{j} .
\end{aligned}
$$

fazendo $\nabla_{X_{i}} X_{j}=\sum_{k} \Gamma_{i j}^{k} X_{k}$, decorre que $\nabla_{X} Y=\sum_{k}\left(\sum_{i j} x_{i} y_{j}+X\left(y_{k}\right)\right) X_{k}$ donde concluimos que $\nabla_{X} Y(p)$ depende de $x_{i}(p)$ e $y_{j}(p)$ e das derivadas $X\left(y_{k}\right)(p)$ de $y_{k}$ segundo $X$

Definição 1.20 Seja $M^{m}$ uma variedade diferenciável com uma conexão afim $\nabla$. Um campo $X$ ao longo de uma curva $\gamma: I \rightarrow M^{m}$ é chamado paralelo quando $\frac{D V}{d t}=0$

Definição 1.21 Seja $M^{m}$ uma variedade diferenciável com uma conexão afim $\nabla$ e uma métrica riemanniana $\langle$,$\rangle . A conexão é chamada compatível com a métrica \langle$,$\rangle , quando para toda curva$ diferenciável $\gamma$ e quaisquer pares de campos de vetores paralelos $X$ e $Y$ ao longo de $\gamma$, tivermos $\langle X, Y\rangle=c$, uma constante.

Teorema 1.22 Uma conexão afim $\nabla$ em uma variedade riemanniana $M^{m}$ é compatível com a métrica se e somente se $X\langle Y, Z\rangle=\left\langle\nabla_{X} Y, Z\right\rangle+\left\langle Y, \nabla_{X} Z\right\rangle$, para todo $X, Y, Z \in \mathfrak{X}(M)$

Definição 1.23 Uma conexão $\nabla$ afim em uma variedade riemanniana $M^{m}$ é chamada simétrica quando

$$
\nabla_{X} Y-\nabla_{Y} X=[X, Y]
$$

para todo $X, Y \in \mathfrak{X}(M)$.

Considerando um sistema de coordenadas $\psi$, pela simetria da conexão $\nabla$

$$
\nabla_{X_{i}} X_{j}-\nabla_{X_{j}} X_{i}=\left[X_{i}, Y_{j}\right]=0 \text { onde } X_{i}=\frac{\partial}{\partial x_{i}}
$$

é equivalente a $\Gamma_{i j}^{k}=\Gamma_{j i}^{k}$.

Teorema 1.24 Dada uma variedade riemanniana $M^{m}$, existe uma única conexão afim $\nabla$ em $M^{m}$ satisfazendo as condições:

1. $\nabla$ é simétrica.

2. $\nabla$ é compatível com a métrica riemanniana. 


\section{Prova:}

Suponhamos que existe uma conexão $\nabla$, então

1. $X\langle Y, Z\rangle=\left\langle\nabla_{X} Y, Z\right\rangle+\left\langle Y, \nabla_{X} Z\right\rangle$

2. $Y\langle Z, X\rangle=\left\langle\nabla_{Y} Z, X\right\rangle+\left\langle Z, \nabla_{Y} X\right\rangle$

3. $Z\langle X, Y\rangle=\left\langle\nabla_{Z} X, Y\right\rangle+\left\langle X, \nabla_{Z} Y\right\rangle$

somando as expressões (1) e (2) e subtraindo (3) decorre que

$$
\begin{gathered}
X\langle Y, Z\rangle+Y\langle Z, X\rangle-Z\langle X, Y\rangle \\
=\langle[X, Z], Y\rangle+\langle[Y, Z], X\rangle+\langle[X, Y], Z\rangle+2\left\langle Z, \nabla_{Y} X\right\rangle .
\end{gathered}
$$

portanto

$$
\begin{aligned}
\left\langle Z, \nabla_{Y} X\right\rangle & =\frac{1}{2}\{X\langle Y, Z\rangle+Y\langle Z, X\rangle-Z\langle X, Y\rangle \\
& -\langle[X, Z], Y\rangle-\langle[Y, Z], X\rangle-\langle[X, Y], Z\rangle\} .
\end{aligned}
$$

mostrar que $\nabla$ está unicamente determinada pela métrica $\langle$,$\rangle .$

Para mostrar a existência da conexão, basta definir como sugerido acima depois mostrar que está bem definida, simétrica e compatível.

Considerando um sistema de coordenadas locais, $\nabla_{X_{i}} X_{j}=\sum_{k} \Gamma_{i j}^{k} X_{k}$ são os coeficientes da conexão $\nabla$. Se $g_{i j}=\left\langle X_{i}, X_{j}\right\rangle$ temos:

$$
\sum_{l} \Gamma_{i j}^{l} g_{l k}=\frac{1}{2}\left\{\frac{\partial}{\partial x_{i}} g_{j k}+\frac{\partial}{\partial x_{j}} g_{k i}-\frac{\partial}{\partial x_{k}} g_{i j}\right\}
$$

como $g_{k m}$ admite inversa $g^{k m}$, temos

$$
\Gamma_{i j}^{m}=\frac{1}{2} \sum_{k}\left\{\frac{\partial}{\partial x_{i}} g_{j k}+\frac{\partial}{\partial x_{j}} g_{k i}-\frac{\partial}{\partial x_{k}} g_{i j}\right\} g^{k m}
$$

\subsection{Geodésica, Vizinhança Normal, Curvaturas}

Definição 1.25 Seja $M^{m}$ uma variedade riemanniana munida com sua conexão riemanniana. Uma curva parametrizada $\gamma: I \rightarrow M^{m}$ é uma geodésica em $t \in I$ se

$$
\frac{D}{d t}\left(\frac{d \gamma}{d t}\right)=0
$$

Em geometria riemanniana sabemos que dado um ponto $p \in M^{m}$, existem uma vizinhança $V$ de $p$ em $M^{m}$, um número $\varepsilon>0$ e uma aplicação $\gamma:(-\varepsilon, \varepsilon) \times \mathcal{U} \rightarrow M$, onde $\mathcal{U}=\{(q, w) \in T M ; q \in$ $\left.V, w \in T_{q} M\right\}$ tal que

$$
t \mapsto \gamma(t, q, w)
$$

$t \in(-\varepsilon, \varepsilon)$, é a única geodésica de $M^{m}$ que no instante $t=0$ passa pelo ponto $q$ com velocidade $w$, para todo $q \in V$ e cada $w \in T_{q} M, \operatorname{com}|w|<\varepsilon$. 


\subsection{Aplicação Exponencial}

Nesse contexto, podemos definir a aplicação exponencial exp $: \mathcal{U} \rightarrow M$ dada por

$$
\exp (q, v)=\gamma(1, q, v)=\gamma\left(|v|, q, \frac{v}{|v|}\right)
$$

onde $(q, v) \in \mathcal{U}$ e $\exp (V)=\mathcal{U}$ é chamada vizinhança normal em $q$. Para $M^{m}$ variedade compacta e $T M$ o fibrado tangente de $M$.

$$
\exp : T M \rightarrow M
$$

é de classe $C^{\infty}$ e existe uma constante $r>0$ tal que

$$
\left.\exp _{x}\right|_{B(0, r)}: B(0, r) \rightarrow B_{g}(x, r)
$$

é um difeomorfismo para todo $x \in M$. neste caso dizemos que $\exp (B(0, r))=B(x, r)$ é a bola normal ou geodésica de centro $x$ e raio $r$.

Fato 1.26 Considere um atlas em $M$, onde as cartas são dadas pela aplicação exponencial, $\mathcal{C}:=$ $\left\{\left(B(q, r), \exp _{q}^{-1}\right)\right\}$, para qualquer função $u \in H_{g}^{1}(M)$.

$$
\int_{M}\left|\nabla_{g} u(x)\right|^{2} d \mu_{g}:=\sum_{B \in C} \int_{B} \psi_{B}(x)\left|\nabla_{g} u(x)\right|^{2} d \mu_{g}
$$

onde $\mathcal{P}=\{\psi: M \rightarrow \mathbb{R}\}$ é uma partição da unidade subordinada ao atlas $\mathcal{C}$. Além disso, se $\operatorname{supp}(u) \subseteq B\left(x_{0}, r\right)$, então

$$
\begin{aligned}
& \int_{B\left(x_{0}, r\right)}\left|\nabla_{g} u(x)\right|^{2} d \mu_{g}= \\
& \int_{B(0, r)}\left(\sum_{i, j=1}^{m} g_{x_{0}}^{i j}(z) \frac{\partial u\left(\exp _{x_{0}}(z)\right)}{\partial z_{i}} \frac{\partial u\left(\exp _{x_{0}}(z)\right)}{\partial z_{j}}\right)\left|g_{x_{0}}(z)\right|^{1 / 2} d z_{1} \ldots d z_{m}
\end{aligned}
$$

onde $g_{x_{0}}$ é uma métrica riemanniana em $B(0, r)$, usando as coordenadas normais definidas pela $\exp _{x_{0}}, g_{x_{0}}(0)=I d,\left|g_{x_{0}}(z)\right|:=\operatorname{det}\left(g_{i j}(z)\right), g^{i j}(z)$ é o inverso de $g_{x_{0}}(z)$.

Seja $r_{0}$ um número positivo tal que para qualquer $r<r_{0}$ e qualquer $x \in M$ a bola geodésica $B(x, r) \subset(M, g)$ é fortemente convexa,isto é, para todo par de pontos $p, p^{\prime} \in B(x, r)$ existe uma única geodésica minimal, $\gamma$, ligando $p$ a $p^{\prime}$, totalmente contida em $B(x, r)$, Pela compacidade de $M$ e a positividade da métrica existem constantes positivas $0<\lambda \leq \Lambda$ tais que

$$
\forall x \in M, \forall v \in T_{x} M, \lambda\|v\|^{2} \leq g_{x}(v, v) \leq \Lambda\|v\|^{2}
$$

implica que $\lambda^{m} \leq\left|g_{x}\right| \leq \Lambda^{m}$

\subsection{Curvaturas}

Definição 1.27 Uma curvatura $R$ de uma variedade riemanniana $M$ com conexão $\nabla$, é uma regra que associa a cada par $X, Y \in \mathfrak{X}(M)$ uma aplicação

$$
R(X, Y): \mathfrak{X}(M) \rightarrow \mathfrak{X}(M)
$$


dada por

$$
R(X, Y) Z=\nabla_{Y} \nabla_{X} Z-\nabla_{X} \nabla_{Y} Z+\nabla_{[X, Y]} Z
$$

onde $Z \in \mathfrak{X}(M)$ e $\nabla$ é a conexão riemanniana de $M$.

Proposição 1.28 (Primeira Identidade de Bianchi)

$$
R(X, Y) Z+R(Y, Z) X+R(Z, X) Y=0
$$

Definição 1.29 Dado um ponto $p \in M$ e um subespaço bidimensional $\pi \subset T_{p} M$, o valor

$$
K(x, y) \equiv K(\pi)=\frac{\langle R(x, y) x, y\rangle}{|x \wedge y|^{2}}
$$

é chamada curvatura seccional de $\pi$ em $p$, onde $x, y$ é uma base qualquer de $\pi$ e $|x \wedge y|^{2}=$ $|x|^{2}|y|^{2}-\langle x, y\rangle^{2}$.

Definição 1.30 Seja $x=z_{m} \in T_{p} M,|x|=1$, considere uma base $\left\{z_{1}, \cdots, z_{m}\right\}$ do hiperplano de $T_{p} M$ ortogonal a $x$, então

$$
\begin{gathered}
\operatorname{Ric}(x)=\sum_{i}\left\langle R\left(x, z_{i}\right) x, z_{i}\right\rangle \\
S(p)=\sum_{j} \operatorname{Ric}\left(z_{j}\right)=\frac{1}{m(m-1)} \sum_{i j}\left\langle R\left(z_{i}, z_{j}\right) z_{i}, z_{j}\right\rangle,
\end{gathered}
$$

são chamadas respectivamente Curvatura de Ricci na direção de $x$ e Curvatura escalar em $p$.

Essas expressões de curvatura independem da escolha da base ortonormal, de fato, considere uma aplicação traço $Q(x, y)=\operatorname{Tr}(z \longmapsto R(x, y) y)$ e obsereve que $\operatorname{Ric}(x)=Q(x, x)$, com relação a curvatura escalar $S(p)$ basta definir como $\langle K(x), y\rangle=Q(x, y)$ onde $k$ é uma aplicação auto-adjunta. Em uma base ortonormal podemos escrever a curvatura escalar como

$$
S_{g}=\sum_{i j k} g^{i k} R_{i j k}^{j}
$$

\subsection{Gradiente, Divergente e Laplaciano}

Definição 1.31 Seja $M$ uma variedade riemanniana e $f \in C^{\infty}(M)$, o gradiente de $f$ é o campo vetorial em $M$, denotado $\nabla f$, tal que

$$
\langle\nabla f(p), v\rangle=d f_{p}(v), p \in M, v \in T_{p} M .
$$

Visto como uma aplicação definida no conjunto $C^{\infty}(M)$, o gradiente é linear e satisfaz a regra de Leibniz, ou seja, dados $f, g \in C^{\infty}(M)$ teremos $\nabla(f+g)=\nabla f+\nabla g$ e $\nabla(f \cdot g)=f \cdot \nabla g+g \cdot \nabla f$. Considere um sistema de coordenadas $\varphi$ em $M$ e uma função $f \in C^{\infty}(M)$, podemos escrever gradiente localmente como

$$
\nabla f=\sum_{i j} g^{i j} \frac{\partial f}{\partial x_{j}} \frac{\partial}{\partial x_{i}}
$$


de fato, suponha que localmente $\nabla f=\sum_{i} a_{i} \frac{\partial}{\partial x_{i}}$, decorre que

$$
\left\langle\nabla f, \frac{\partial}{\partial x_{j}}\right\rangle=\sum_{i} a_{i}\left\langle\frac{\partial}{\partial x_{i}}, \frac{\partial}{\partial x_{j}}\right\rangle=\sum_{i} a_{i} g_{i j}
$$

implica que $a_{i}=\sum_{j} g^{i j}\left\langle\nabla f, \frac{\partial}{\partial x_{j}}\right\rangle=\sum_{j} g^{i j} d f\left(\frac{\partial}{\partial x_{j}}\right)=\sum_{j} g^{i j} \frac{\partial f}{\partial x_{j}}$, portanto $\nabla f=\sum_{i j} g^{i j} \frac{\partial f}{\partial x_{j}} \frac{\partial}{\partial x_{i}}$.

Consequentemente para $u, v \in C^{\infty}(M)$

$$
\langle\nabla u, \nabla v\rangle=\sum_{i, j} g^{i j} \frac{\partial u}{\partial x_{i}} \frac{\partial v}{\partial x_{j}}
$$

Definição 1.32 Seja $X \in \mathfrak{X}(M)$, chamamos de divergente de $X$ a aplicação $\operatorname{div} X: M \rightarrow \mathbb{R}$ definida da seguinte forma

$$
\operatorname{div} X(p)=\operatorname{Tr}\left(\begin{array}{ccc}
T_{p} M & \longrightarrow & T_{p} M \\
v & \longmapsto & \left(\nabla_{v} X\right)(p)
\end{array}\right)
$$

Decorre que $\operatorname{div}(X+Y)=\operatorname{div} X+\operatorname{div} Y$ e $\operatorname{div}(f \cdot X)=f \cdot \operatorname{div} X+\langle\nabla f, X\rangle$, para dois campos quaisquer $X, Y \in \mathfrak{X}(M)$ e $f \in C^{\infty}(M)$.

Considere um sistema de coordenadas $\varphi$, e um campo $X \in \mathfrak{X}(M)$, nessa carta $X=\sum_{j} a_{j} \frac{\partial}{\partial x_{j}}$, por definição $\operatorname{div} X(p)=\operatorname{Tr}\left(\left(\nabla_{v} X\right)(p)\right)$ observe que nessa condições temos

$$
\begin{aligned}
\nabla_{v} X & =\nabla_{\left(\frac{\partial}{\partial x_{i}}\right)}\left(\sum_{j} a_{j} \frac{\partial}{\partial x_{j}}\right) \\
& =\sum_{j}\left(a_{j} \nabla \frac{\partial}{\partial x_{i}} \frac{\partial}{\partial x_{j}}+\frac{\partial a_{j}}{\partial x_{i}} \frac{\partial}{\partial x_{j}}\right) \\
& =\sum_{j}\left(\sum_{k} a_{j} \Gamma_{i j}^{k} \frac{\partial}{\partial x_{j}}+\frac{\partial a_{j}}{\partial x_{i}} \frac{\partial}{\partial x_{j}}\right) \\
& =\sum_{k}\left(\frac{\partial a_{k}}{\partial x_{i}}+\sum_{j} a_{j} \Gamma_{i j}^{k}\right) \frac{\partial}{\partial x_{k}}
\end{aligned}
$$

donde concluímos que $\operatorname{div} X=\sum_{i}\left(\frac{\partial a_{i}}{\partial x_{i}}+\sum_{j} a_{j} \Gamma_{i j}^{i}\right)$

Definição 1.33 O operador de Laplace-Beltrami é definido como

$$
-\Delta f=\operatorname{div}(\nabla f)
$$

onde $f \in C^{\infty}(M)$

Lema 1.34 Nas hipótese acima, temos que $\Delta f=\frac{-1}{\sqrt{\operatorname{det}\left(g_{i j}\right)}} \sum_{i j}\left(\sqrt{\operatorname{det}\left(g_{i j}\right)} \cdot g^{i j} \frac{\partial f}{\partial x_{j}}\right)$, onde $f \in$ $C^{\infty}(M)$. 
Definição 1.35 (Submersão Riemaniana) $\left(M^{m}, g_{M}\right)$ e $\left(B^{n}, g_{B}\right)$ variedades riemannianas, $\pi$ : $M \rightarrow B$ uma submersão, $M, B$ e $\mathcal{F}$ são chamados respectivamente de espaço total, base e fibra de $\pi$. Pela forma local das submersões $\mathcal{F}=\pi^{-1}(\pi(q))$ é uma subvariedade riemanniana mergulhada de $M$ de dimensão $m-n$ tal que

$$
T_{q} \mathcal{F}=\operatorname{Ker}\left(d \pi_{q}\right)
$$

$d \pi_{q}$ é sobrejetiva, sua restrição a $T_{q} \mathcal{F}^{\perp}$ induz um isomorfismo

$$
\left(d \pi_{q}\right): T_{q} \mathcal{F}^{\perp} \rightarrow T_{\pi(q)} B
$$

Um vetor é vertical se for tangente a uma fibra e um campo é vertical se for inteiramente composto de vetores verticais. Um vetor é horizontal se for ortogonal a uma fibra e um campo é horizontal se for inteiramente composto de vetores horizontais. Fixado $q \in M$, denotamos por $H_{q}$ e $V_{q}$ os conjuntos de vetores tangentes a $M$ em $q$ e respectivamente horizontais, verticais. Segue que $H_{q}$ e $V_{q}$ são subespaços vetoriais de $T_{q} M$ e que vale a decomposição em soma direta ortogonal

$$
T_{q} M=H_{q} \oplus V_{q}
$$

\subsection{Geometria Conforme}

Nesta seção vamos reescrever as expressões de conexão riemaniana e curvatura considerando uma métrica conforme a $g$, que definiremos a seguir, para tanto considere $\left(M^{m}, g\right)$ uma variedade Riemaniana de dimensão $m \geq 3$ e $\psi$ um sistema de coordenadas.

Definição 1.36 Sejam $g$ e $\tilde{g}$ métricas Riemannianas definidas em $M^{m}$. Dizemos que $\tilde{g}$ é conforme a $g$, quando existe uma função $\phi \in C^{\infty}(M)$ positiva tal que $\tilde{g}=\phi g$. Denotamos $[g]$ a classe conforme de uma metrica $g$.

Sem perca de generalidade usaremos $\tilde{g}=e^{2 f} g$, onde $f \in C^{\infty}(M)$, como representante da classe $[g]$, localmente $\tilde{g}=\tilde{g}_{i j} d x^{i} \otimes d x^{j}=e^{2 f} g_{i j} d x^{i} \otimes d x^{j}$, portanto $\tilde{g}_{i j}=e^{2 f} g_{i j}$ e sua inversa é $\tilde{g}^{i j}=e^{-2 f} g^{i j}$.

Seja $\nabla$ a conexão riemanniana em $T M$ associada à métrica $g$ da variedade $M^{m}$, seja $\tilde{\nabla}$ a conexão riemanniana associada à métrica conforme $\tilde{g}=e^{2 f} g$. Considere $X, Y, Z \in \mathfrak{X}(M)$ campos vetoriais suaves em $M^{m}$. Usando a fórmula de Koszul e em seguida a regra de Leibniz temos: 


$$
\begin{aligned}
2 \tilde{g}\left(\tilde{\nabla}_{X} Y, Z\right) & =X \tilde{g}(Y, Z)+Y \tilde{g}(X, Z)-Z \tilde{g}(Y, X) \\
& -\tilde{g}([X, Y], Z)-\tilde{g}([X, Z], Y)-\tilde{g}([Y, Z], X) \\
& =e^{2 f}[2 X(f) g(Y, Z)+X g(Y, Z)+2 Y g(X, Z)+Y g(X, Z) \\
& -2 Z(f) g(X, Y)-Z g(X, Y)-g([X, Y], Z)-g([X, Z], Y)-g([Y, Z], X)] \\
& =e^{2 f}[2 X(f) g(Y, Z)+2 Y(f) g(X, Z)-2 Z(f) g(X, Y)] \\
& +e^{2 f}[X g(Y, Z)+Y g(X, Z)-Z g(X, Y) \\
& -g([X, Y], Z)-g([X, Z], Y)-g([Y, Z], X)] \\
& =e^{2 f}[X(f) g(Y, Z)+Y(f) g(X, Z)-Z(f) g(X, Y)] \\
& +e^{2 f}\left[2 g\left(\nabla_{X} Y, Z\right)\right] \\
& \left(\operatorname{considere~} Z(f)=g(\nabla f, Z) \text { e } \nabla f:=\operatorname{grad}_{g} f\right) \\
& =2\left[X(f) e^{2 f} g(Y, Z)+Y(f) e^{2 f} g(X, Z)-e^{2 f} g(\nabla f, Z) g(X, Y)\right] \\
& +2 e^{2 f} g\left(\nabla_{X} Y, Z\right)
\end{aligned}
$$

Decorre que

$$
\begin{aligned}
\tilde{g}\left(\tilde{\nabla}_{X} Y, Z\right) & =[X(f) \tilde{g}(Y, Z)+Y(f) \tilde{g}(X, Z)-Z(\nabla f, Z) g(X, Y)]+\tilde{g}\left(\nabla_{X} Y, Z\right) \\
& =\tilde{g}(X(f) Y, Z)+\tilde{g}(Y(f) X, Z)-\tilde{g}(g(X, Y) \nabla f, Z)+\tilde{g}\left(\nabla_{X} Y, Z\right) \\
& =\tilde{g}\left(X(f) Y+Y(f) X-g(X, Y) \nabla f+\nabla_{X} Y, Z\right)
\end{aligned}
$$

para qualquer campo $Z \in \mathfrak{X}(M)$, Portanto

$$
\tilde{\nabla}_{X} Y=\nabla_{X} Y+X(f) Y+Y(f) X-g(X, Y) \nabla f
$$

\subsubsection{Curvatura Riemanniana Conforme}

Vamos calcular a curvatura riemanniana com respeito à métrica conforme $\tilde{g}$, por definição

$$
\tilde{R}(X, Y) Z=\tilde{\nabla}_{X} \tilde{\nabla}_{Y} Z-\tilde{\nabla}_{Y} \tilde{\nabla}_{X} Z-\tilde{\nabla}_{[X, Y]} Z,
$$

usando o fato demonstrado anteriormente $\tilde{\nabla}_{Y} Z=\nabla_{Y} Z+Y(f) Z+Z(f) Y-g(Y, Z) \nabla f$,

Vamos computar cada parcela de $\tilde{R}(X, Y) Z$, assim temos 


$$
\begin{aligned}
\tilde{\nabla}_{X} \tilde{\nabla}_{Y} Z & =\tilde{\nabla}_{X} \nabla_{Y} Z+\tilde{\nabla}_{X} Y(f) Z+\tilde{\nabla}_{X} Z(f) Y-\tilde{\nabla}_{X} g(Y, Z) \nabla f \\
& =\nabla_{X} \nabla_{Y} Z+X(f) \nabla_{Y} Z+\nabla_{Y} Z(f) X-g\left(X, \nabla_{Y} Z\right) \nabla f \\
& +X Y(f) Z+Y(f) \tilde{\nabla}_{X} Z+X Z(f) Y+Z(f) \tilde{\nabla}_{X} Y \\
& -X g(Y, Z) \nabla f-g(Y, Z) \tilde{\nabla}_{X}(\nabla f) \\
& =\nabla_{X} \nabla_{Y} Z-X(f) \nabla_{Y} Z+\nabla_{Y} Z(f) X-g\left(X, \nabla_{Y} Z\right) \nabla f \\
& +X Y(f) Z+Y(f) \nabla_{X} Z+Y(f) X(f) Z+Y(f) Z(f) X-Y(f) g(X, Z) \nabla f \\
& +X Z(f) Y+Z(f) \nabla_{X} Y+Z(f) X(f) Y+Z(f) Y(f) X-Z(f) g(X, Y) \nabla f \\
& -X g(Y, Z) \nabla f-g(Y, Z)\left[\nabla_{X}(\nabla f)+X(f) \nabla f+\nabla f(f) X-g(X, \nabla f) \nabla f\right] \\
& =\nabla_{X} \nabla_{Y} Z-X(f) \nabla_{Y} Z+\nabla_{Y} Z(f) X-g\left(X, \nabla_{Y} Z\right) \nabla f \\
& +X Y(f) Z+Y(f) \nabla_{X} Z+Y(f) X(f) Z+Y(f) Z(f) X-Y(f) g(X, Z) \nabla f \\
& +X Z(f) Y+Z(f) \nabla_{X} Y+Z(f) X(f) Y+Z(f) Y(f) X-Z(f) g(X, Y) \nabla f \\
& -X g(Y, Z) \nabla f-g(Y, Z) \operatorname{hess}_{f}(X)-g(Y, Z)\|\nabla f\|^{2} X .
\end{aligned}
$$

$$
\begin{aligned}
\tilde{\nabla}_{Y} \tilde{\nabla}_{X} Z & =\nabla_{Y} \nabla_{X} Z-Y(f) \nabla_{X} Z+\nabla_{X} Z(f) Y-g\left(Y, \nabla_{X} Z\right) \nabla f \\
& +Y X(f) Z+X(f) \nabla_{Y} Z+X(f) Y(f) Z+X(f) Z(f) Y-X(f) g(Y, Z) \nabla f \\
& +Y Z(f) X+Z(f) \nabla_{Y} X+Z(f) Y(f) X+Z(f) X(f) Y-Z(f) g(Y, X) \nabla f \\
& -Y g(X, Z) \nabla f-g(X, Z) \operatorname{hess}_{f}(Y)-g(X, Z)\|\nabla f\|^{2} Y . \\
& \tilde{\nabla}_{[X, Y]} Z=\nabla_{[X, Y]} Z+[X, Y](f) Z+Z(f)[X, Y]-g([X, Y], Z) \nabla f,
\end{aligned}
$$

implica em

$$
\begin{aligned}
\tilde{\nabla}_{[X, Y]} Z & =\nabla_{[X, Y]} Z+[X, Y](f) Z+Z(f)[X, Y]-g([X, Y], Z) \nabla f \\
& =\nabla_{[X, Y]} Z+X(Y(f)) Z-Y(X(f)) Z+Z(f) \nabla_{X} Y-Z(f) \nabla_{Y} X \\
& -g\left(\nabla_{X} Y, Z\right) \nabla f+g\left(\nabla_{Y} X, Z\right) \nabla f .
\end{aligned}
$$

em particular $\nabla_{X, Y}^{2}=X(Y f)-\left(\nabla_{X} Y\right) f$, agrupando os termos adequadamente e usando a compatibilidade da metrica com a conexão teremos

$$
\begin{aligned}
\tilde{R}(X, Y) Z & =R(X, Y) Z \\
& -\left[\nabla^{2} f(Y, Z)-Y(f) Z(f)+g(Y, Z)\|\nabla f\|^{2}\right] X \\
& +\left[\nabla^{2} f(X, Z)-X(f) Z(f)+g(X, Z)\|\nabla f\|^{2}\right] Y \\
& -g(Y, Z) \operatorname{hess}_{f}(X)-g(X, Z) \operatorname{hess}_{f}(Y) \\
& -[Y(f) g(X, Z)-X(f) g(Y, Z)] \nabla f .
\end{aligned}
$$

\subsubsection{Curvatura de Ricci Conforme}

Considere um referencial ortonormal $\left\{e_{i}\right\}_{i=1}^{m}$ em $T M$ com respeito à métrica $g$, podemos definir um referencial ortonormal com respeito a métrica $\tilde{g}$ em $T M$ dado por $\tilde{e}_{i}=e^{-f} e_{i}$. A curvatura de 
Ricci de $M^{m}$ para a métrica $\tilde{g}$ é:

$$
\begin{aligned}
\operatorname{Ric}_{\tilde{g}}(Y, Z) & =\sum_{i=1}^{m} \tilde{g}\left(\tilde{R}\left(\tilde{e}_{i}, Y\right) Z, \tilde{e}_{i}\right) \\
& =\sum_{i=1}^{m} e^{2 f} g\left(\tilde{R}\left(e^{-f} e_{i} Y\right) Z, e^{-f} e_{i}\right) \\
& =e^{2 f} e^{-2 f} \sum_{i=1}^{m} g\left(\tilde{R}\left(e_{i}, Y\right) Z, e_{i}\right) \\
& =\sum_{i=1}^{m} g\left(\tilde{R}\left(e_{i}, Y\right) Z, e_{i}\right) \\
& =\sum_{i=1}^{m} g\left(R\left(e_{i}, Y\right) Z\right. \\
& -\left[\nabla^{2} f(Y, Z)-Y(f) Z(f)+g(Y, Z)\|\nabla f\|^{2}\right] e_{i} \\
& +\left[\nabla^{2} f\left(e_{i}, Z\right)-e_{i}(f) Z(f)+g\left(e_{i}, Z\right)\|\nabla f\|^{2}\right] Y \\
& -g(Y, Z) \operatorname{hess}_{f}\left(e_{i}\right)-g\left(e_{i}, Z\right) \operatorname{hess} f(Y) \\
& \left.-\left[Y(f) g\left(e_{i}, Z\right)-e_{i}(f) g(Y, Z)\right] \nabla f\right)
\end{aligned}
$$

Vamos fazer alguns cálculos visando simplificar a expressao do Ricci de $\tilde{g}$ :

$\sum_{i} g\left(\tilde{R}\left(e_{i}, Y\right) Z, e_{i}\right)=\operatorname{Ric}_{g}(Y, Z)$

$\sum_{i} g\left(g\left(e_{i}, Z\right)\|\nabla f\|^{2} Y, e_{i}\right)=\sum_{i} g\left(e_{i}, Z\right)\|\nabla f\|^{2} g\left(Y, e_{i}\right)=g(Y, Z)\|\nabla f\|^{2}$

$\sum_{i} g\left(g(Y, Z) \operatorname{hess}_{f}\left(e_{i}\right), e_{i}\right)=\sum_{i} g(Y, Z) g\left(\operatorname{hess}_{f}\left(e_{i}\right), e_{i}\right)=g(Y, Z) \operatorname{tr}_{g} \operatorname{hess}_{f}=-g(Y, Z) \Delta f$

$\sum_{i} g\left(g\left(e_{i}, Z\right) \operatorname{hess}_{f}(Y), e_{i}\right)=\sum_{i} g\left(e_{i}, Z\right) g\left(\operatorname{hess}_{f}(Y), e_{i}\right)=g\left(\operatorname{hess}_{f}(Y), Z\right)=\nabla^{2} f(Y, Z)$

$\sum_{i} g\left(Y(f) g\left(e_{i}, Z\right) \nabla f, e_{i}\right)=\sum_{i} Y(f) g\left(e_{i}, Z\right) g\left(\nabla f, e_{i}\right)=Y(f) g(\nabla f, Z)=Y(f) Z(f)$.

$$
\begin{aligned}
\sum_{i} g\left(\nabla^{2} f\left(e_{i}, Z\right) Y, e_{i}\right) & =\sum_{i} \nabla^{2} f\left(e_{i}, Z\right) g\left(Y, e_{i}\right) \\
& =\sum_{i} g\left(\operatorname{hess}_{g} f\left(e_{i}\right), Z\right) g\left(Y, e_{i}\right) \\
& =\sum_{i} g\left(\operatorname{hess}_{g} f\left(e_{i}\right), Z\right) Y_{i} \\
& =\sum_{i} g\left(\operatorname{hess}_{g} f\left(e_{i}\right) Y_{i}, Z\right) \\
& =g\left(\operatorname{hess}_{f}(Y), Z\right) \\
& =\nabla^{2} f(Y, Z)
\end{aligned}
$$




$$
\begin{aligned}
\sum_{i} g\left(e_{i}(f) Z(f) Y, e_{i}\right) & =\sum_{i} e_{i}(f) Z(f) Y g\left(Y, e_{i}\right) \\
& =\sum_{i} g\left(\nabla f, e_{i}\right) g(\nabla f, Z) g\left(Y, e_{i}\right) \\
& =g(\nabla f, Y) g(\nabla f, Z) \\
& =Y(f) Z(f) \\
\sum_{i} g\left(e_{i}(f) g(Y, Z) \nabla f, e_{i}\right) & =\sum_{i} e_{i}(f) g(Y, Z) g\left(\nabla f, e_{i}\right) \\
& =\sum_{i} g\left(\nabla f, e_{i}\right) g(Y, Z) g\left(\nabla f, e_{i}\right) \\
& =g(Y, Z) g(\nabla f, \nabla f) \\
& =g(Y, Z)\|\nabla f\|^{2}
\end{aligned}
$$

Portanto, usando as simplicações feitas anteriormente, podemos reescrever o Ricci de $\tilde{g}$

$$
\begin{aligned}
\operatorname{Ric}_{\tilde{g}}(Y, Z) & =\operatorname{Ric}_{g}(Y, Z)-m \nabla^{2} f(Y, Z)+m Y(f) Z(f)-m g(Y, Z)\|\nabla f\|^{2} \\
& +\nabla^{2} f(Y, Z)-Y(f) Z(f)+g(Y, Z)\|\nabla f\|^{2} \\
& +g(Y, Z) \Delta f+\nabla^{2} f(Y, Z)-Y(f) Z(f)+g(Y, Z)\|\nabla f\|^{2} \\
& =\operatorname{Ric}_{g}(Y, Z)-(m-2) \nabla^{2} f(Y, Z)+(m-2) Y(f) Z(f) \\
& -(m-2) g(Y, Z)\|\nabla f\|^{2}+g(Y, Z) \Delta f .
\end{aligned}
$$

\subsubsection{Curvatura Escalar Conforme}

A curvatura escalar da métrica $\tilde{g}$ é o traço do tensor de Ricci para $\tilde{g}$, calculado acima:

$$
R_{\tilde{g}}=\sum_{i} \operatorname{Ric}_{\tilde{g}}\left(\tilde{e}_{i}, \tilde{e}_{i}\right),
$$

onde $\tilde{e}_{i}$ é o referencial ortonormal com respeito à $\tilde{g}$. Decorre

$$
\begin{aligned}
R_{\tilde{g}} & =\sum_{i} \operatorname{Ric}_{\tilde{g}}\left(e^{-f} e_{i}, e^{-f} e_{i}\right) \\
& =e^{-2 f} \sum_{i} \operatorname{Ric}_{\tilde{g}}\left(e_{i}, e_{i}\right) \\
& =e^{-2 f} \sum_{i}\left[\operatorname{Ric}_{g}\left(e_{i}, e_{i}\right)-(m-2) \nabla^{2} f\left(e_{i}, e_{i}\right)+(m-2) e_{i}(f) e_{i}(f)\right] \\
& \left.-(m-2) g\left(e_{i}, e_{i}\right)\|\nabla f\|^{2}+g\left(e_{i}, e_{i}\right) \Delta f\right] \\
& =e^{-2 f}\left[R_{g}+(m-2) \Delta f+(m-2)\|\nabla f\|^{2}-(m-2) m\|\nabla f\|^{2}+m \Delta f\right] \\
& =e^{-2 f}\left[R_{g}+2(m-1) \Delta f-(m-2)(m-1)\|\nabla f\|^{2}\right]
\end{aligned}
$$




\section{Capítulo 2}

\section{Os Espaços de Sobolev}

\subsection{O Espaço de Sobolev $W^{1, p}(\Omega)$}

Sejam $M^{m}$ uma variedade riemanniana e $\varphi: U \rightarrow M$ um sistema de coordenadas, digamos que $\Omega=x(U)$. Usaremos a notação $G$ para indicar o determinante da matriz $\left(g_{i j}\right)_{m}$

Definição 2.1 Seja $u \in L^{1}(\Omega)$ e $f \in L^{1}(\Omega)$ será a derivada fraca na direção de $x_{k}$ de $u$, denotada por $\partial x_{k}$, (no sentido das distribuições) em $\Omega=x(U)$, quando

$$
\int_{\Omega} \frac{u}{\sqrt{G}} \frac{\partial v}{\partial x_{k}} d V=-\int_{\Omega} f \frac{u}{\sqrt{G}} d V,
$$

para todo $v \in C_{0}^{\infty}(\Omega), \operatorname{supp} v \subset \subset \Omega$ e independe da escolha de parametrização.

Lema 2.2 Seja $u \in L^{1}(\Omega)$. Então, u possui derivada $\partial x_{k}$ fraca em $\Omega$ se, e somente se, $u \circ x$ possui derivada fraca $\partial_{k}(u \circ x)$ em $U$. Mais ainda, se $f$ é a sua derivada $\partial x_{k}$ fraca em $\Omega$, então $f \circ x: U \rightarrow \mathbb{R}$ é a derivada fraca $\partial_{k}(u \circ x)$ da função $u \circ x$.

\section{Prova:}

Seja $\phi \in C_{0}^{\infty}(U)$. Considere $\psi \in C^{\infty}(\Omega)$ dada por $\psi=\phi \circ x^{-1}$. Temos que $\operatorname{supp} \psi \subset \subset \Omega$, decorre que $(\psi \circ x)(z)=\phi(z)$ consequentemente

$$
\partial_{k} \phi(z)=\partial_{k}(\psi \circ x)(z)=\frac{\partial \psi}{\partial x_{k}}(x(z))
$$

para todo $z \in U$. Por definição,

$$
\begin{aligned}
\int_{U}(u \circ x) \partial_{k} \phi(z) d z & =\int_{U}(u \circ x) \frac{\partial_{k}(\psi \circ x)}{\sqrt{G \circ x}} \sqrt{G \circ x} d z \\
& =\int_{\Omega} \frac{u}{\sqrt{G}} \frac{\partial \psi}{\partial x_{k}} d V=-\int_{\Omega} \frac{f \psi}{\sqrt{G}} d V \\
& =\int_{U}(f \circ x) \frac{(\psi \circ x)}{\sqrt{G \circ x}} \sqrt{G \circ x} d z=-\int_{U}(f \circ x) d z
\end{aligned}
$$


Definição 2.3 Seja $u \in L^{1}(\Omega)$. Suponha que $u$ possui todas as derivadas $\partial x_{k}$ fracas em $\Omega=x(U)$, denotadas por $f_{k}: V \rightarrow \mathbb{R}, k=1,2, \ldots, n$. O gradiente fraco de $u$ em $\Omega=x(U)$ é o campo

$$
\operatorname{grad}(u)=\sum_{i, j=1}^{n} g^{i j}(p) f_{i}(p) \frac{\partial}{x_{j}}, p \in x(U) .
$$

usaremos $\operatorname{grad}(u)=\nabla u$, quando $u \in C^{\infty}(\Omega)$.

Lema 2.4 Suponha que $u \in L^{1}(\Omega)$ possui gradiente fraco em $\Omega$. Então para todo $v \in C^{\infty}(\Omega)$, suppv $\subset \subset \Omega$, temos

$$
\int_{\Omega}\langle\operatorname{grad}(u), \nabla v\rangle d V=-\int_{\Omega} u \Delta v d V .
$$

\section{Prova:}

por 1.7, temos

$$
\langle\operatorname{grad}(u), \nabla v\rangle=\sum_{i, j=1}^{n} g^{i j}(p) f_{i}(p) \frac{\partial v}{x_{j}},
$$

onde $f_{i}$ é a derivada $\partial_{x_{i}}$ fraca de $u$.

Utilizando 1.34, juntamente com a definição dos $f_{k}$, temos

$$
\begin{aligned}
\int_{\Omega} u \Delta v d V & =\sum_{i, j=1}^{n} \int_{\Omega} \frac{u}{\sqrt{G}} \frac{\partial}{\partial x_{i}}\left[g^{i j} \sqrt{G} \frac{\partial v}{\partial x_{j}}\right] d V \\
& =-\sum_{i, j=1}^{n} \int_{\Omega} \frac{f_{i} g^{i j}}{\sqrt{G}} \sqrt{G} \frac{\partial v}{\partial x_{j}} d V \\
& =-\sum_{i, j=1}^{n} \int_{\Omega} f_{i} g^{i j} \frac{\partial v}{\partial x_{j}} d V \\
& =-\int_{\Omega}\left[\sum_{i, j=1}^{n} g^{i j} f_{i} \frac{\partial v}{\partial x_{j}}\right] d V \\
& =-\int_{\Omega}\langle\operatorname{grad}(u), \nabla v\rangle d V .
\end{aligned}
$$

Além disso, denotaremos

$$
\operatorname{grad}(u)=\sum_{i, j=1}^{n} g^{i j}(p) \frac{\partial u}{\partial x_{i}}(p) \frac{\partial u}{\partial x_{j}}, p \in x(U)
$$

e

$$
\langle\operatorname{grad}(u), \operatorname{grad}(u)\rangle=\sum_{i, j=1}^{n} g^{i j} \frac{\partial u}{\partial x_{i}} \frac{\partial u}{\partial x_{j}} .
$$


Considere o sistema de coordenadas $x: U \rightarrow \Omega \operatorname{com} U$ aberto limitado e tal que é satisfeito 1.3

Definição 2.5 O espaço vetorial $W^{1, p}(\Omega)=\left\{u \in L^{p}(\Omega):|\operatorname{grad}(u)| \in L^{p}(\Omega)\right\}$ munido com a norma

$$
\|u\|_{W^{1, r}(\Omega)}=\left(\int_{\Omega}|u|^{r} d V+\int_{\Omega}|\operatorname{grad}(u)|^{r} d V\right)^{\frac{1}{r}}
$$

é o espaço de Sobolev $W^{1, p}$ sobre a vizinhança parametrizada $\Omega$. Quando $p=2, H^{1}(\Omega)=W^{1,2}(\Omega)$ é um espaço de Hilbert munido do produto interno

$$
\langle u, v\rangle_{H^{1}(\Omega)}=\int_{\Omega} u v d V+\int_{\Omega}\langle\operatorname{grad}(u), \operatorname{grad}(v)\rangle d V .
$$

decorre de 1.3 e 2.4 , que

$$
\lambda^{p}\left(\sum_{i=1}^{m}\left|\partial_{i}(u \circ x)(z)\right|^{2}\right)^{\frac{p}{2}} \leq|\operatorname{grad}(u)|^{p}(q) \leq \Lambda^{p}\left(\sum_{i=1}^{m}\left|\partial_{i}(u \circ x)(z)\right|^{2}\right)^{\frac{p}{2}}
$$

para todo $z \in U$ e $q=x(z)$. Usando 1.3 implica

$$
\lambda^{p+1} \int_{U}\left[\sum_{i=1}^{m}\left|\partial_{i}(u \circ x)(z)\right|^{2}\right]^{\frac{p}{2}} d z \leq \int_{\Omega}|\operatorname{grad}(u)|^{r} d V \leq \Lambda^{p+1} \int_{U}\left[\sum_{i=1}^{m}\left|\partial_{i}(u \circ x)(z)\right|^{2}\right]^{\frac{p}{2}} d z
$$

Lema 2.6 A aplicação $\pi: W^{1, p}(\Omega) \rightarrow W^{1, p}(U)$, dada por $\pi(u)=u \circ x$ é um isomorfismo. As normas $\|\left. u\right|_{W^{1, p}(\Omega)}$ e $|u|_{\Omega, 1, p}=\|u\|_{W^{1, p}(U)}$ são equivalentes.

Lema 2.7 (Desigualdade de Poincaré) Existe uma constante $C=C_{r, \Omega}>0$ tal que

$$
\int_{\Omega}|v|^{r} d V \leq C \int_{\Omega}|\operatorname{grad}(v)|^{r} d V
$$

para todo $v \in W^{1, r}(\Omega)$, com $\operatorname{supp}(v) \subset \subset \Omega$.

O gradiente fraco independe da parametrização escolhida, considere as parametrizações $x_{\alpha} \mathrm{e}$ $x_{\beta}$ tais que $x_{\alpha}\left(U_{\alpha}\right)=(\Omega)=x_{\beta}\left(U_{\beta}\right)$. Suponha que haja dois gradientes fracos para uma função $u \in L^{1}(\Omega)$, a saber:

$$
\begin{aligned}
& \operatorname{grad}_{\alpha}(u)=\sum_{i, j=1}^{n} g^{i j}(p) \frac{\partial u}{\partial x_{i}}(p) \frac{\partial u}{\partial x_{j}}, p \in \Omega \\
& \operatorname{grad}_{\beta}(u)=\sum_{k, l=1}^{n} s^{k l}(p) \frac{\partial u}{\partial y_{k}}(p) \frac{\partial u}{\partial y_{l}}, p \in \Omega
\end{aligned}
$$


decorre que

$$
\begin{aligned}
\operatorname{grad}_{\alpha}(u) & =\sum_{i, j=1}^{m}\left[\sum_{k, l=1}^{m} \frac{\partial x_{i}}{\partial y_{l}}(q) s^{l k} \frac{\partial x_{j}}{\partial y_{k}}(q)\right] \frac{\partial u}{\partial x_{i}}(p) \frac{\partial u}{\partial x_{j}} \\
& =\sum_{k, l=1}^{m} s^{l k}\left[\sum_{i, j=1}^{m} \frac{\partial x_{i}}{\partial y_{l}}(q) \frac{\partial x_{j}}{\partial y_{k}}(q) \frac{\partial u}{\partial x_{i}}(p) \frac{\partial u}{\partial x_{j}}\right] \\
& =\sum_{k, l=1}^{m} s^{l k}\left[\sum_{i=1}^{m} \frac{\partial u}{\partial x_{i}}(p) \frac{\partial x_{i}}{\partial y_{l}}(q)\right]\left[\sum_{j=1}^{m} \frac{\partial x_{j}}{\partial y_{k}}(q) \frac{\partial u}{\partial x_{j}}\right]
\end{aligned}
$$

donde

$$
\operatorname{grad}_{\alpha}(u)=\sum_{k, l=1}^{n} s^{l k}(p) \frac{\partial u}{\partial y_{k}}(p) \frac{\partial}{\partial y_{l}}=\operatorname{grad}_{\beta}(u)
$$

Lema 2.8 O gradiente fraco não depende da parametrização escolhida, ou seja, se $x_{\alpha}$ e $x_{\beta}$ são duas parametrizações tais que $x_{\alpha}\left(U_{\alpha}\right)=(\Omega)=x_{\beta}\left(U_{\beta}\right)$ então para uma função $u \in L^{1}(\Omega)$ onde está definida o gradiente fraco $\operatorname{grad}_{\alpha}(u)$, também existe o gradiente fraco $\operatorname{grad}_{\beta}(u)$ e deveremos ter

$$
\operatorname{grad}_{\alpha}(u)=\operatorname{grad}_{\beta}(u) .
$$

\subsection{O Espaço de Sobolev $W^{1, p}(M)$}

Definição 2.9 Seja $u \in L^{1}(M)$. Suponha que para qualquer vizinhança parametrizada $x_{\alpha}: U \rightarrow$ $\Omega_{\alpha}$ o gradiente fraco $\operatorname{grad}_{\alpha}(u)$ está definido. Então o campo vetorial definido por $\operatorname{grad}(u)=$ $\operatorname{grad}_{\alpha}(u)$ em $\Omega_{\alpha}$ é chamado de gradiente fraco de $u$ em $M$.

Pelo lema 2.7, temos a boa definição e além disso a função $u \in L^{1}(M)$ possui um gradiente fraco. Certamente que $\operatorname{grad}(u)=\nabla u$, quando $u \in C^{\infty}(M)$. Decorre de modo geral que:

Lema 2.10 Suponha que $u \in L^{1}(M)$ possui gradiente fraco em $\Omega$. Então para todo $v \in C^{\infty}(M)$, temos

$$
\int_{M}\langle\operatorname{grad}(u), \nabla v\rangle d V=-\int_{M} u \Delta v d V
$$

Definição 2.11 O espaço vetorial $W^{1, p}(M)=\left\{u \in L^{p}(M):|\nabla u| \in L^{p}(M)\right\}$ munido com a norma

$$
\|u\|_{W^{1, p}(M)}=\left(\int_{M}|u|^{p} d V+\int_{M}|\nabla u|^{p} d V\right)^{\frac{1}{p}}
$$

é o espaço de Sobolev $W^{1, p}$ sobre a variedade $M$. Quando $p=2, H^{1}(M)=W^{1,2}(M)$ é um espaço de Hilbert munido do produto interno

$$
\langle u, v\rangle_{H^{1}(M)}=\int_{M} u v d V+\int_{M}\langle\nabla u, \nabla v\rangle d V .
$$


Lema 2.12 As normas $\|u\|_{W^{1, p}(M)} e$

$$
\|u\|_{1, p}^{*}=\left[\sum_{n=1}^{m}\|u\|_{\Omega_{\alpha}, 1, p}^{p}\right]^{\frac{1}{p}}=\left[\sum_{n=1}^{m}\left\|u \circ x_{\alpha}\right\|_{W^{1, p}\left(U_{\alpha}\right)}^{r}\right]^{\frac{1}{p}}
$$

são equivalentes em $W^{1, p}(M)$. Aqui, denotamos $|u|_{\Omega_{\alpha}, 1, p}^{p}=\left\|u \circ x_{\alpha}\right\|_{W^{1, p}\left(U_{\alpha}\right)}^{p}$.

Fato 2.13 1. Se $u \in W^{1, p}(M), v \in L^{\infty}(M)$ e $f: \mathbb{R} \rightarrow \mathbb{R}$ é de classe $C^{1}(\mathbb{R})$ tal que $\left|f^{\prime}(t)\right| \leq C$, $\forall t \in \mathbb{R}$, então $v=f \circ u \in W^{1, p}(M)$ e $\nabla v=f^{\prime}(u) \nabla u$.

2. Se $u \in W^{1, p}(M)$ e $\psi \in C^{1}(M)$ então $u \psi \in W^{1, p}(M)$ e $\nabla(u \psi)=\psi \nabla u+u \nabla \psi$.

3. Se $u, v \in W^{1, p}(M)$ e $u, v \in L^{\infty}(M)$ então $u v \in W^{1, p}(M)$ e $\nabla(u v)=v \nabla u+u \nabla v$.

\subsection{As imersões de Sobolev sobre a Variedade $M$}

Teorema 2.14 (Imersões de Sobolev para variedade compacta) Seja $M$ uma variedade compacta de dimensão $m$. Valem as seguintes afirmações:

$i$ - Se $\frac{1}{s} \geq \frac{1}{p}-\frac{1}{m}$, então $W^{1, p}(M)$ está imerso continuamente em $L^{s}(M)$;

ii - (Rellich-Kondrachov) A imersão acima é compacta se $\frac{1}{s}>\frac{1}{p}-\frac{1}{m}$.

Fato 2.15 Decorre que existe uma menor constante $S_{M}$ tal que

$$
S_{M}\left(\int_{M}|u|^{r} d V\right)^{\frac{2}{2^{*}}} \leq \int_{M}\left(|\nabla u|^{2}+u^{2}\right) d V
$$

para todo $u \in H^{1}(M)$. Aqui $2^{*}=\frac{2 m}{m-2}$.

Fato 2.16 Se $u \in W^{1, p}(M) \cap L^{\infty}(M)$, então para cada $s^{\prime}>1$, temos $|u|^{s} \in W^{1, p}(M), \frac{\partial}{\partial x_{k}}\left(|u|^{s}\right)=$ $s|u|^{s-2} u \frac{\partial}{\partial x_{k}}$ e consequentemente, $\nabla\left(|u|^{s}\right)=s|u|^{s-2} u \nabla u$.

Proposição 2.17 Sejam $Q \in L^{\frac{m}{2}}(M)$ uma função não negativa e $f: M \times \mathbb{R} \rightarrow \mathbb{R}$ uma função de Carathéodory. Se $v \in H^{1}(M)$ satisfaz em $M$, no sentido das distribuições, a equação

$$
-\delta v=f(p, v)
$$

e f verifica, para toda solução fraca u de 2.8.

$$
|f(p, u)| \leq\left(Q(p)+C_{0}\right)|u|
$$

onde $C_{0}$ é uma constante, então $v \in L^{r}(M)$ para todo $r \in[1,+\infty)$. Mais ainda, existe uma constante positiva $C_{p}=C\left(p, C_{0}, Q\right)$ tal que

$$
\|v\|_{L^{2^{*}(p+1)}(M)} \leq C_{p}\|v\|_{L^{2(p+1}(M)} .
$$




\subsection{Um problema Elíptico não linear em variedade riemanniana}

Seja $\left(M, g_{M}\right)$ uma variedade riemaniana compacta, conexa de classe $C^{\infty}, m=\operatorname{dim} M \geq 3$. Vamos inicialmente observar o seguinte problema elíptico não linear;

$$
\left\{\begin{array}{c}
-\varepsilon^{2} \Delta_{g} u+u=u|u|^{p-2} \\
u>0
\end{array}\right.
$$

onde $\Delta_{g}$ é o operador de Laplace-Beltrami com $u \in H_{g_{M}}^{1}(M)$ onde

$$
H_{g_{M}}^{1}(M):=\left\{u: M \rightarrow \mathbb{R}: \int_{M}\left(|\nabla u(x)|^{2}+|u(x)|^{2}\right) d \mu_{g_{M}}<\infty\right\}
$$

e $p=\frac{2 m}{m-2}$ é o expoente crítico de Sobolev.

Seja $E_{\varepsilon}: H^{1}(M) \rightarrow \mathbb{R}$ o $C^{2}$-funcional definido como

$$
E_{\varepsilon}(u)=\frac{1}{\varepsilon^{m}} \int_{M}\left(\frac{1}{2} \varepsilon^{2}|\nabla u(x)|^{2}+\frac{1}{2}|u(x)|^{2}-\frac{1}{p}\left(u^{+}(x)\right)^{p}\right) d \mu
$$

onde $u^{+}(x)=\max (u(x), 0)$.

Definição 2.18 A variedade de Nehari associada ao funcional $E_{\varepsilon}$ é

$$
N\left(E_{\varepsilon}\right)=\left\{u \in H^{1}(M) \backslash\{0\}: \int_{M}\left(\varepsilon^{2}|\nabla u(x)|^{2}+|u(x)|^{2}\right) d \mu_{g_{M}}=\int_{M}\left(u^{+}(x)\right)^{p}, u \neq 0\right\}
$$

os pontos críticos do funcional $E_{\varepsilon}$ em $N\left(E_{\varepsilon}\right)$ são soluções para a equação 2.11

O mínimo de $E_{\varepsilon}$ é:

$$
\mathrm{m}\left(E_{\varepsilon}\right)=\min \left\{E_{\varepsilon}(u) ; u \in N\left(E_{\varepsilon}\right)\right\}
$$

O subnível do funcional $E_{\varepsilon}$ é

$$
\left.\sum_{\mathrm{m}\left(E_{\varepsilon}\right), \delta}=\left\{u \in N\left(E_{\varepsilon}\right) ; E_{\varepsilon}(u)<\mathrm{m}\left(E_{\varepsilon}\right)+\delta\right)\right\}
$$

Considere agora o funcional $E: H^{1}\left(\mathbb{R}^{m}\right) \rightarrow \mathbb{R}$

$$
E(v)=\int_{\mathbb{R}^{m}}\left(\frac{1}{2}|\nabla v(x)|^{2}+\frac{1}{2}|v(x)|^{2}-\frac{1}{p}\left(v^{+}(x)\right)^{p}\right) d x
$$

a variedade de Nehari para $E$ é $N(E)$

$$
N(E)=\left\{v \in H^{1}\left(\mathbb{R}^{m}\right): \int_{\mathbb{R}^{m}}\left(|\nabla v|^{2}+|v|^{2}\right) d x=\int_{\mathbb{R}^{m}}\left(v^{+}\right)^{p} d x, u \neq 0\right\}
$$

Lema 2.19 A variedade de Nehari $N(E)$ é não vazia.

\section{Prova:}

Seja $u \in H_{0}^{1}(\Omega)$, então para $\lambda=\left(\frac{\|u\|_{H_{0}^{1}(\Omega)}^{2}}{\int_{\Omega}|u|^{p} d x}\right)^{\frac{1}{p-2}}>0$, temos que $\lambda u \in N(E)$. 
O mínimo é

$$
\mathrm{m}(E)=\inf \{E(v): v \in N(E)\}=\left(\frac{1}{2}-\frac{1}{p}\right)\|U\|_{p}^{p}
$$

Fato $2.20 \mathrm{~m}(E)=\inf \{E(v): v \in N(E)\}>0$, de fato, como $u \in N(E)$ existe uma constante $\sigma>0$, tal que

$$
\|u\|_{H_{0}^{1}(\Omega)}^{2}=\int_{\Omega}|u|^{p} d x \leq \sigma\|u\|_{H_{0}^{1}(\Omega)}^{p}
$$

Decorre que

$$
\|u\|_{H_{0}^{1}(\Omega)}^{2} \geq\left(\frac{1}{\sigma}\right), \forall u \in N(E)
$$

portanto

$$
m \geq\left(\frac{1}{2}-\frac{1}{p}\right)\left(\frac{1}{\sigma}\right)>0 .
$$

Por Gidas, Ni e Nirenberg em [10] existe uma única função positiva radialmente simétrica (em relação a origem) $U \in H^{1}\left(\mathbb{R}^{m}\right)$ tal que $E(U)=\mathrm{m}(E)$, consequentemente $U$ satisfaz a equação

$$
\left\{\begin{array}{l}
-\Delta U+U=U^{p-1} \\
U>0
\end{array}\right.
$$

uma solução fraca para a equação 2.4 é uma função $u \in H_{0}^{1}(\Omega)$ tal que, para todo $v \in H_{0}^{1}(\Omega)$

$$
\int_{\Omega}\langle\nabla u, \nabla v\rangle+\int_{\Omega} u v=\int_{\Omega} u v|u|^{p-1}
$$

por Vieri Benci e Giovanna Cerami em [4] observaram que para $\varepsilon>0$ podemos definir uma familia de soluções $U_{\varepsilon}(x)=U\left(\frac{x}{\varepsilon}\right)$ safisfazendo a seguinte equação:

$$
\left\{\begin{array}{c}
-\varepsilon^{2} \Delta U_{\varepsilon}+U_{\varepsilon}=U_{\varepsilon}^{p_{m}-1} \\
U_{\varepsilon}>0
\end{array}\right.
$$

Decorre das contribuições de Gidas, Ni e Nirenberg que a função $U$ é exponencialmente decrescente no infinito, pois $U(|x|)|x|^{\frac{m-1}{2}} e^{|x|}=c_{1}$ e $U^{\prime}(|x|) e^{\frac{m-1}{2}} e^{|x|}=c_{2}$ quando $|x| \rightarrow \infty$ com $c_{1}$ e $c_{2}$ constantes positivas, $U_{\varepsilon}$ é um minimizador de $E_{\varepsilon}$ restrito a $N\left(E_{\varepsilon}\right)$ e $\mathrm{m}(E)=\mathrm{m}\left(E_{\varepsilon}\right)$

Considere uma sequência minimizante $\left(u_{k}\right)_{k} \in N(E)$, tal que $u_{k}(x) \neq 0$ quase sempre em $\Omega \subset \mathbb{R}^{m}$. Como $E$ é coerciva, $\left(u_{k}\right)_{k}$ é limitada em $H^{1}(\Omega)$. Pelas desigualdades de Sobolev temos que a subsequência

$$
\left\{\begin{array}{cc}
u_{k} \rightarrow u & \text { em } H_{0}^{1}(\Omega) \\
u_{k} \rightarrow u & \text { em } L^{p}(\Omega) \\
\mathrm{e} & \\
u_{k}(x) \rightarrow u & \text { quase sempre em } \Omega
\end{array}\right.
$$


então $u>0$ quase sempre, pela semi-continuidade de $E$ temos

$$
E(u) \leq \lim \inf E\left(u_{k}\right)=\mathrm{m}(E)
$$

temos pelo lema 2.19 que $\|u\|_{H_{0}^{1}(\Omega)} \nrightarrow 0 \Rightarrow \int_{\Omega}\left|u_{k}\right|^{p} d x \nrightarrow 0$, pela convergencia forte

$$
\int_{\Omega}\left|u_{k}\right| d x \neq 0 \Rightarrow u \neq 0
$$

no limite teremos que

$$
\|u\|_{H_{0}^{1}(\Omega)} \leqslant \int_{\Omega}\left|u_{k}\right|^{p} d x
$$

Suponhamos que $\|u\|_{H_{0}^{1}(\Omega)}<\int_{\Omega}\left|u_{k}\right|^{p} d x$, considere $\lambda$, como no lema 2.19, que implica $\lambda u \in$ $N(E)$ e $0<\lambda<1$, então

$$
0<\mathrm{m}(E)<E(\lambda u)=\lambda^{2}\left(\frac{1}{2}-\frac{1}{p}\right)\|u\|_{H_{0}^{1}(\Omega)}^{2} \leq \lambda^{2}\left(\frac{1}{2}-\frac{1}{p}\right)\left\|u_{k}\right\|_{H_{0}^{1}(\Omega)}^{2}=\lambda^{2} m<m
$$

uma contradição. portanto temos que existe $u \in N(E)$ positiva, tal que $E(u)=\mathrm{m}(E)$ 


\section{Capítulo 3}

\section{Problema de Yamabe - subcrítico}

No ano de 1960 o japonês Hidehiko Yamabe, conjecturou que para toda variedade riemanniana compacta de dimensão maior ou igual a 3, sempre existe um métrica conforme com curvatura escalar constante, foi completamente resolvido em 1984 com contribuições de Yamabe, Trundinger, Aubin e Shoen. Hoje essa conjectura é conhecida como Problema de Yamabe.

\subsection{Problema de Yamabe}

Definição 3.1 Sejam $g$ e $\tilde{g}$ métricas Riemannianas em $N^{n}$. Dizemos que $\tilde{g}$ é conforme a $g$, quando existe uma função $\phi \in C^{\infty}(N)$ positiva tal que $\tilde{g}=\phi g$.

O próximo teorema apresenta um modelo no contexto de equações elípticas para o problema de Yamabe, verificaremos através de alguns cálculos que a curvaturas escalar $S_{g}$ esta relacionada com curvatura escalar da métrica conforme correspondente.

Teorema 3.2 Se g e $\tilde{g}$ são métricas conformes em uma variedade Riemanniana $N$, onde assumiremos que $\tilde{g}=u^{\frac{4}{n-2}} g$, onde $u \in C^{\infty}(N), u>0$. Então

$$
\Delta_{g} u+\frac{n-2}{4(n-1)} u S_{g}=\frac{n-2}{4(n-1)} u^{\frac{n+2}{n-2}} S_{\tilde{g}}
$$

é o modelo que relaciona $S_{g}$ e $S_{\tilde{g}}$.

\section{Prova:}

Considere um sistema de coordenadas $x: U \rightarrow N^{n}$, expressando $\tilde{g}$ nesse sistema, temos $\tilde{g}_{i j}=$ $u^{r} g_{i j}$ e $\tilde{g}^{i j}=u^{-r} g^{i j}$ onde $r=\frac{4}{n-2}$. Usando a expressão dos símbolos de Christoffel no sistema de coordenadas $x$ e fazendo $\partial_{i}=\frac{\partial}{\partial x_{i}}$, decorre que 


$$
\begin{aligned}
\tilde{\Gamma}_{i j}^{l} & =\frac{1}{2} \sum_{k}\left(\partial_{i} \tilde{g}_{j k}+\partial_{j} \tilde{g}_{i k}-\partial_{k} \tilde{g}_{i j}\right) \tilde{g}^{k l} \\
& =\frac{1}{2} \sum_{k}\left[\partial_{i}\left(u^{r} g_{j k}\right)+\partial_{j}\left(u^{r} g_{i k}\right)-\partial_{k}\left(u^{r} g_{i j}\right)\right] u^{-r} g^{k l} \\
& =\frac{1}{2} r u^{-1} \sum_{k}\left(g_{j k} \partial_{i} u+g_{i k} \partial_{j} u-g_{i j} \partial_{k} u\right) g^{k l}+\frac{1}{2} \sum_{k}\left(\partial_{i} g_{j k}+\partial_{j} g_{i k}-\partial_{k} g_{i j}\right) g^{k l} \\
& =\Gamma_{i j}^{l}+\frac{1}{2} r u^{-1}\left[\sum_{k}\left(g_{k j}^{j k} \partial_{i} u\right)+\sum_{k}\left(g_{i k} g^{k l} \partial_{j} u\right)-\sum_{k}\left(g_{i j} g^{k l} \partial_{k} u\right)\right] \\
& =\Gamma_{i j}^{l}+\frac{1}{2} r u^{-1}\left[\delta_{j i} \partial_{i} u+\delta_{i l} \partial_{j} u-\sum_{k}\left(g_{i j} g^{k l} \partial_{k} u\right)\right]
\end{aligned}
$$

A curvatura escalar $S_{\tilde{g}}$ é dada por

$$
S_{\tilde{g}}=\sum_{i j k} \tilde{g}^{i k} \tilde{R}_{i j k}^{j}
$$

implica

$$
S_{\tilde{g}}=\sum_{i j k} u^{-r} g^{i k}\left[\partial_{j} \tilde{\Gamma}_{j k}^{j}+\sum_{s} \tilde{\Gamma}_{i k}^{s} \tilde{\Gamma}_{j s}^{j}-\sum_{s} \tilde{\Gamma}_{j k}^{s} \tilde{\Gamma}_{i s}^{j}\right]
$$

Substituindo $\tilde{\Gamma}_{i j}^{l}$ em $S_{\tilde{g}}$ teremos:

$$
\begin{aligned}
u^{r} S_{\tilde{g}} & =\sum_{i j k} g^{i k} \partial_{j}\left[\Gamma_{i k}^{j}+\frac{1}{2} r u^{-1}\left(\delta_{k j} \partial_{i} u+\delta_{i j} \partial_{k} u-\sum_{m} g_{i k} g^{m j} \partial_{m} u\right)\right]+ \\
& -\sum_{i j k} g^{i k} \partial_{i}\left[\Gamma_{j k}^{j}+\frac{1}{2} r u^{-1}\left(\delta_{k j} \partial_{j} u+\delta_{j j} \partial_{k} u-\sum_{m} g_{j k} g^{m j} \partial_{m} u\right)\right]+ \\
& \sum_{i j k s} g^{i k}\left[\Gamma_{i k}^{s}+\frac{1}{2} r u^{-1}\left(\delta_{k s} \partial_{i} u+\delta_{i s} \partial_{k} u-\sum_{m} g_{i k} g^{m s} \partial_{m} u\right)\right] \\
& \cdot\left[\Gamma_{j s}^{j}+\frac{1}{2} r u^{-1}\left(\delta_{s j} \partial_{j} u+\delta_{j j} \partial_{s} u-\sum_{p} g_{j s} g^{p j} \partial_{p} u\right)\right]+ \\
& -\sum_{i j k s} g^{i k}\left[\Gamma_{j k}^{s}+\frac{1}{2} r u^{-1}\left(\delta_{k s} \partial_{j} u+\delta_{j s} \partial_{k} u-\sum_{m} g_{j k} g^{m s} \partial_{m} u\right)\right] \\
& \cdot\left[\Gamma_{i s}^{j}+\frac{1}{2} r u^{-1}\left(\delta_{s j} \partial_{i} u+\delta_{i j} \partial_{s} u-\sum_{l} g_{i s} g^{l j} \partial_{l} u\right)\right]
\end{aligned}
$$


Vamos agora detalhar cada parcela da expressão $u^{r} S_{\tilde{g}}$ e verificar se é possivel relacioná-la com alguma estrutura já conhecida

$$
\begin{aligned}
Y .1 & =\sum_{i j k} g^{i k} \partial_{j} \Gamma_{i k}^{j}+\frac{r u^{-1}}{2} \sum g^{i k} \delta_{k j} \partial_{j i} u+\frac{r u^{-1}}{2} \sum_{i j k} g^{i k} \delta_{i j} \partial_{j k} u+ \\
& -\frac{r u^{-1}}{2} \sum_{i j k m} g^{i k} \partial_{j}\left(g_{i k} g^{m j} \partial_{m} u\right)-\frac{r u^{-2}}{2} \sum_{i j k} g^{i k} \delta_{k j} \partial_{j} u \partial_{i} u+ \\
& -\frac{r u^{-2}}{2} \sum_{i j k} g^{i k} \delta_{i j} \partial_{j} u \partial_{k} u+\frac{r u^{-2}}{2} \sum_{i j k m} g^{i k} g_{i k} \partial_{j} u \partial_{m} u \\
& =\sum_{i j k} g^{i k} \partial_{j} \Gamma_{i k}^{j}+\frac{r u^{-1}}{2} \sum_{i k} g^{i k} \partial_{k i} u+\frac{r u^{-1}}{2} \sum_{j k} g^{j k} \partial_{j k} u+ \\
& -\frac{r u^{-1}}{2} \sum_{i j k m} g^{i k} \partial_{j}\left(g_{i k} g^{m j} \partial_{m} u\right)-\frac{r u^{-2}}{2} \sum_{j k} g^{j k} \partial_{j} u \partial_{k} u+ \\
& -\frac{r u^{-2}}{2} \sum_{j k} g^{j k} \partial_{j} u \partial_{k} u+\frac{n r u^{-2}}{2} \sum_{m j} g^{m j} \partial_{j} u \partial_{m} u \\
& =\sum_{i j k} g^{i k} \partial_{j} \Gamma_{i k}^{j}+r u^{-1} \sum_{i k} g^{i k} \partial_{k i} u-\frac{r u^{-1}}{2} \sum_{i j k m} g^{i k} \partial_{j}\left(g_{i k}\right) g^{m j} \partial_{m} u+ \\
& -\frac{r u^{-1}}{2} \sum_{i j k m} g^{i k} g_{i k} \partial_{j}\left(g^{m j}\right) \partial_{m} u-\frac{r u^{-1}}{2} \sum_{i j k m} g^{i k} g_{i k} g^{m j} \partial_{m j} u+ \\
& +\frac{(n-2) r u^{-2}}{2} \sum_{j k} g^{j k} \partial_{j} u \partial_{k} u \\
& =\sum_{i j k} g^{i k} g_{j} \Gamma_{i k}^{j}+r u_{j} \Gamma_{i k}^{j} \sum_{i k} g^{i k} \partial_{k i} u-\frac{r u^{-1}}{2} \sum_{i j k m} g^{i k} \partial_{j}\left(g_{i k}\right) g^{m j} \partial_{m} u+ \\
& \frac{n r u^{-1}}{2} \sum_{j m} \partial_{j}\left(g^{m j}\right) \partial_{m} u-\frac{n r u^{-1}}{2} \sum_{j m} g^{m j} \partial_{m j} u+2 \partial_{k i} u-\frac{r u^{-1}}{2} \sum_{i j k m} g_{j k} g^{j k} \partial_{j}\left(g_{i k}\right) g^{m j} \partial_{j} u \partial_{k} u \\
& \\
& \\
& \\
&
\end{aligned}
$$


onde $\partial_{j}\left(g^{m j}\right)=-\sum_{i k} g^{m i} \partial_{j}\left(g_{i k}\right) g^{j k}$, agora vamos para a segunda parcela de $u^{r} S_{\tilde{g}}$

$$
\begin{aligned}
Y .2 & =\sum_{i j k} g^{i k} \partial_{j} \Gamma_{j k}^{j}+\sum_{i j k} g^{i k} \partial_{i}\left[\frac{r u^{-1}}{2}\left(\delta_{k j} \partial_{j} u+\delta_{j j} \partial_{k} u-\sum_{m} g_{j k} g^{m j} \partial_{m} u\right)\right] \\
& =\sum_{i j k} g^{i k} \partial_{j} \Gamma_{j k}^{j}+\sum_{i k} g^{i k} \partial_{i}\left[\sum_{j} \frac{n r u^{-1}}{2}\left(\delta_{k j} \partial_{j} u+\delta_{j j} \partial_{k} u-\sum_{m} g_{j k} g^{m j} \partial_{m} u\right)\right] \\
& =\sum_{i j k} g^{i k} \partial_{i} \Gamma_{j k}^{j}+\frac{n r}{2} \sum_{i k} g^{i k} \partial_{i}\left(u^{-1} \partial_{k} u\right) \\
& =\sum_{i j k} g^{i k} \partial_{i} \Gamma_{j k}^{j}-\frac{n r u^{-2}}{2} \sum_{i k} g^{i k} \partial_{i} u \partial_{k} u+\frac{n r u^{-1}}{2} \sum_{i k} g^{i k} \partial_{i k} u .
\end{aligned}
$$

a terceira parcela de $u^{r} S_{\tilde{g}}$

$$
\begin{aligned}
Y .3 & =\sum_{i j k s} g^{i k} \Gamma_{i k}^{s}+\Gamma_{j s}^{j} \frac{r u^{-1}}{2} \sum_{i j k s} g^{i k} \Gamma_{i k}^{s}\left(\delta_{s j} \partial_{j} u+\delta_{j j} \partial_{s} u-\sum_{p} g_{j s} g^{p j} \partial_{p} u\right)+ \\
& +\frac{r u^{-1}}{2} \sum_{i j k s} g^{i k} \Gamma_{j s}^{j}\left(\delta_{k s} \partial_{i} u+\delta_{i s} \partial_{k} u-\sum_{m} g_{i k} g^{m s} \partial_{m} u\right) \\
& +\frac{r^{2} u^{-2}}{4} \sum_{i j k s} g^{i k}\left(\delta_{k s} \partial_{i} u+\delta_{i s} \partial_{k} u-\sum_{m} g_{i k} g^{m s} \partial_{m} u\right) \\
& \cdot\left(\delta_{s j} \partial_{j} u+\delta_{j j} \partial_{s} u-\sum_{p} g_{j s} g^{p j} \partial_{p} u\right) \\
& =\sum_{i j k s} g^{i k} \Gamma_{i k}^{s} \Gamma_{j s}^{j}+\frac{n r u^{-1}}{2} \sum_{i k s} g^{i k} \Gamma_{i k}^{s} \partial_{s} u+\frac{r u^{-1}}{2} \sum_{i j s} g^{i s} \Gamma_{j s}^{j} \partial_{i} u+ \\
& \frac{r u^{-1}}{2} \sum_{j k s} g^{s k} \Gamma_{j s}^{j} \partial_{k} u-\frac{n r u^{-1}}{2} \sum_{j s m} g^{m s} \Gamma_{j s}^{j} \partial_{m} u+ \\
& \frac{n r^{2} u^{-2}}{4} \sum_{i k s} g^{i k} \delta_{k s} \partial_{i} u \partial_{s} u+\frac{n r^{2} u^{-2}}{4} \sum_{i k s} g^{i k} \delta_{i s} \partial_{k} u \partial_{s} u+ \\
& -\frac{n r^{2} u^{-2}}{4} \sum_{i k s m} g^{i k} g_{i k} g^{m s} \partial_{m} u \partial_{s} u \\
& +\frac{4 n u^{-2}}{2-n} \sum_{k s} g^{k s} \partial_{k} u \partial_{s} u . \\
& +\sum_{i j k s} g^{i k} \Gamma_{i k}^{s}+\Gamma_{j s}^{j}+\frac{n r u^{-1}}{2} \sum_{i k s} g^{i k} \Gamma_{i k}^{s} \partial_{s} u+\frac{(2-n) r u^{-1}}{2} \sum_{j k s} g^{k s} \Gamma_{j s}^{j} \partial_{k} u+ \\
& \left(\frac{n r^{2} u^{-2}}{2}-\frac{n^{2} r^{2} u^{-2}}{4}\right) \sum_{k s} g^{k s} \partial_{k} u \partial_{s} u \\
& \frac{n r u^{-1}}{2} \sum_{j k s} g^{i k} \Gamma_{i k}^{s} \partial_{s} u+2 u^{-1} \sum_{j s}^{k s} \Gamma_{k}^{j} \partial_{k} u \\
& \\
&
\end{aligned}
$$


A última parcela a ser estudada é

$$
\begin{aligned}
& Y .4=\sum_{i j k s} g^{i k} \Gamma_{j k}^{s} \Gamma_{i s}^{j}+\frac{r u^{-1}}{2} \sum_{i j k s} g^{i k} \Gamma_{j k}^{s}\left(\delta_{s j} \partial_{i} u+\delta_{i j} \partial_{s} u-\sum_{l} g_{i s} g^{l j} \partial_{l} u\right)+ \\
& +\frac{r u^{-1}}{2} \sum_{i j k s} g^{i k} \Gamma_{i s}^{j}\left(\delta_{k s} \partial_{j} u+\delta_{j s} \partial_{k} u-\sum_{m} g_{j k} g^{m s} \partial_{m} u\right)+ \\
& \frac{r^{2} u^{-2}}{4} \sum_{i j k s} g^{i k}\left(\delta_{k s} \partial_{j} u+\delta_{j s} \partial_{k} u-\sum_{m} g_{j k} g^{m s} \partial_{m} u\right) \text {. } \\
& \cdot\left(\delta_{s j} \partial_{i} u+\delta_{i j} \partial_{s} u-\sum_{l} g_{i s} g^{l j} \partial_{l} u\right) \\
& =\sum_{i j k s} g^{i k} \Gamma_{j k}^{s} \Gamma_{i s}^{j}+\frac{r u^{-1}}{2} \sum_{i j s} g^{i s} \Gamma_{i s}^{j} \partial_{j} u+\frac{r u^{-1}}{2} \sum_{i k s} g^{i k} \Gamma_{i s}^{s} \partial_{k} u+ \\
& -\frac{r u^{-1}}{2} \sum_{i j k s l} g^{i k} \Gamma_{j k}^{s} g_{i s} g^{j l} \partial_{l} u+\frac{r u^{-1}}{2} \sum_{i j s} g^{i s} \Gamma_{i s}^{j} \partial_{j} u+\frac{r u^{-1}}{2} \sum_{i k s} g^{i k} \Gamma_{i s}^{s} \partial_{k} u+ \\
& -\frac{r u^{-1}}{2} \sum_{i j k s m} g^{i k} \Gamma_{i s}^{j} g_{j k} g^{m s} \partial_{m} u+\frac{r^{2} u^{-2}}{4} \sum_{i j k s} g^{i k} \delta_{k s} \partial_{j} u \delta_{s j} \partial_{i} u \\
& +\frac{r^{2} u^{-2}}{4} \sum_{i j k s} g^{i k} \delta_{k s} \partial_{j} u \delta_{i j} \partial_{s} u-\frac{r^{2} u^{-2}}{4} \sum_{i j k s l} g^{i k} \delta_{k s} \partial_{j} u g_{i s} g^{j l} \partial_{l} u+ \\
& +\frac{r^{2} u^{-2}}{4} \sum_{i j k s} g^{i k} \delta_{j s} \partial_{k} u \delta_{s j} \partial_{i} u+\frac{r^{2} u^{-2}}{4} \sum_{i j k s} g^{i k} \delta_{j s} \partial_{k} u \delta_{i j} \partial_{s} u+ \\
& -\frac{r^{2} u^{-2}}{4} \sum_{i j k s l} g^{i k} \delta_{j s} \partial_{k} u g_{i s} g^{j l} \partial_{l} u+\frac{r^{2} u^{-2}}{4} \sum_{i j k s m} g^{i k} g_{j k} g^{m s} \partial_{m} u \delta_{s j} \partial_{i} u+ \\
& -\frac{r^{2} u^{-2}}{4} \sum_{i j k s m} g^{i k} g_{j k} g^{m s} \partial_{m} u \delta_{i j} \partial_{s} u+\frac{r^{2} u^{-2}}{4} \sum_{i j k s m l} g^{i k} g_{j k} g^{m s} \partial_{m} u g_{i s} g^{j l} \partial_{l} u \\
& =\sum_{i j k s} g^{i k} \Gamma_{j k}^{s} \Gamma_{i s}^{j}+r u^{-1} \sum_{i j k} g^{i k} \Gamma_{i k}^{j} \partial_{j} u+\frac{(2-n) r^{2} u^{-2}}{4} \sum_{i k} g^{i k} \partial_{k} u \partial_{i} u .
\end{aligned}
$$


Ao observar as expressões $Y .1, Y .2, Y .3$ e $Y .4$, percebemos que ao somarmos a primeira parcela de cada uma delas obtemos $S_{g}$ e agora vamos somar os respectivos resultados encontrados

$$
\begin{aligned}
u^{r} S_{\tilde{g}} & =Y .1+Y .2+Y .3+Y .4 \\
& =S_{g}-2 u^{-1} \sum_{i k} g^{i k} \partial_{i k} u-\frac{r u^{-1}}{2} \sum_{i j k m} g^{i k} \partial_{j}\left(g_{i k}\right) g^{m j} \partial_{m} u+ \\
& +\frac{n r u^{-1}}{2} \sum_{i j k m} g^{m i} \partial_{j}\left(g_{i k}\right) g^{k j} \partial_{m} u+2 u^{-2} \sum_{j k} g^{j k} \partial_{j} u \partial_{k} u+ \\
& +\frac{n r u^{-2}}{2} \sum_{i k} g^{i k} \partial_{i} u \partial_{k} u-\frac{n r u^{-1}}{2} \sum_{i k} g^{i k} \partial_{i k} u+\frac{n r u^{-1}}{2} \sum_{i k s} g^{i k} \Gamma_{i k}^{s} \partial_{s} u \\
& -2 u^{-1} \sum_{j k s} g^{k s} \Gamma_{j s}^{j} \partial_{k} u+\frac{4 n u^{-2}}{2-n} \sum_{k s} g^{k s} \partial_{k} u \partial_{s} u-r u^{-1} \sum_{i j k} g^{i k} \Gamma_{i k}^{j} \partial_{j} u+ \\
& -\frac{4 u^{-2}}{2-n} \sum_{k s} g^{k s} \partial_{k} u \partial_{s} u .
\end{aligned}
$$

decorre que

$$
\begin{aligned}
u^{r+1} S_{\tilde{g}} & =u S_{g}-\frac{4(n-1)}{n-2} \sum_{i k} g^{i k} \partial_{i k} u-\frac{r}{2} \sum_{i j k m} g^{i k} \partial_{j}\left(g_{i k}\right) g^{m j} \partial_{m} u+ \\
& +\frac{n r}{2} \sum_{i j k m} g^{m i} \partial_{j}\left(g_{i k}\right) g^{k j} \partial_{m} u+2 \sum_{i j k} g^{i k} \Gamma_{i k}^{j} \partial_{j} u-2 \sum_{j k s} g^{k s} \Gamma_{j s}^{j} \partial_{k} u
\end{aligned}
$$

Considere a expressão dos símbolos de Christoffel em termos de componentes da métrica

$$
\sum_{i j k} g^{i k} \Gamma_{i k}^{j} \partial_{j} u-\sum_{j k s} g^{k s} \Gamma_{j s}^{j} \partial_{k} u=\sum_{j k s l} g^{k s} g^{j l} \partial_{l}\left(g_{j s}\right) \partial_{k} u-\sum_{j k s l} g^{k s} g^{j l} \partial_{s}\left(g_{j l}\right) \partial_{k} u .
$$

Substituindo a sentença anterior em 4.1, obtemos

$$
\begin{aligned}
u^{r+1} S_{\tilde{g}} & =u S_{g}-\frac{4(n-1)}{n-2} \sum_{i k} g^{i k} \partial_{i k} u-\frac{r}{2} \sum_{i j k m} g^{i k} \partial_{j}\left(g_{i k}\right) g^{m j} \partial_{m} u+ \\
& +\frac{n r}{2} \sum_{i j k m} g^{m i} \partial_{j}\left(g_{i k}\right) g^{k j} \partial_{m} u+2 \sum_{j k s l} g^{k s} g^{j l} \partial_{l}\left(g_{j s}\right) \partial_{k} u+ \\
& -2 \sum_{j k s l} g^{k s} g^{j l} \partial_{s}\left(g_{j l}\right) \partial_{k} u .
\end{aligned}
$$

donde

$$
\begin{aligned}
\frac{n-2}{n-1} u^{\frac{n+2}{n-2}} S_{\tilde{g}} & =\frac{n-2}{n-1} u S_{g}-4 \sum_{i k} g^{i k} \partial_{i k} u-2 \sum_{j k s l} g^{k s} g^{j l} \partial_{s}\left(g_{j l}\right) \partial_{k} u+ \\
& +4 \sum_{j k s l} g^{k s} g^{j l} \partial_{l}\left(g_{j s}\right) \partial_{k} u
\end{aligned}
$$


Considere a última parcela do lado direito desta igualdade, podemos reescre-la da seguinte forma

$$
4 \sum_{j k s l} g^{k s} g^{j l} \partial_{l}\left(g_{j s}\right) \partial_{k} u=2 \sum_{j k s l} g^{k s} g^{j l} \partial_{l}\left(g_{j s}\right) \partial_{k} u+2 \sum_{j k s l} g^{k s} g^{j l} \partial_{l}\left(g_{j s}\right) \partial_{k} u
$$

portanto

$$
\begin{aligned}
\frac{n-2}{n-1} u^{\frac{n+2}{n-2}} S_{\tilde{g}} & =\frac{n-2}{n-1} u S_{g}-4 \sum_{i k} g^{i k} \partial_{i k} u+ \\
& +2 \sum_{j k s l} g^{k s} g^{j l}\left[\partial_{l}\left(g_{j s}\right)+\partial_{j}\left(g_{l s}\right)-\partial_{s}\left(g_{j l}\right)\right] \partial_{k} u \\
& =\frac{n-2}{n-1} u S_{g}-4 \sum_{i k} g^{i k} \partial_{i k} u+4 \sum_{j i k} g^{j l} \Gamma_{j l}^{k} \partial_{k} u \\
& =\frac{n-2}{n-1} u S_{g}-4 \sum_{j l} g^{j l}\left(\partial_{j l} u-\sum_{k} \Gamma_{j l}^{k} \partial_{k} u\right) .
\end{aligned}
$$

como

$$
\Delta_{g} u=\sum_{j l} g^{j l}\left(\partial_{j l} u-\sum_{k} \Gamma_{j l}^{k} \partial_{k} u\right) .
$$

implica

$$
\Delta_{g} u+\frac{n-2}{4(n-1)} u S_{g}=\frac{n-2}{4(n-1)} u^{\frac{n+2}{n-2}} S_{\tilde{g}}
$$

\subsection{Problema de Yamabe Subcrítico}

Como vimos na seção anterior o Problema de Yamabe subcrítico consiste em determinar soluções para o problema elíptico seguinte

$$
\Delta_{g} u+\frac{n-2}{4(n-1)} S_{g} u=\mu u^{p-1}
$$

onde $2<p<2 *, \mu \in \mathbb{R}$, vamos considerar o funcional associado ao problema acima $Y: N \rightarrow \mathbb{R}$, definido por

$$
Y(u)=\int_{N}|\nabla u|^{2} d V+\frac{n-2}{4(n-1)} \int_{N} S_{g} u^{2} d V
$$

Além disso $H_{p}=\left\{u \in H^{1}(N) ;\|u\|_{L^{p}(N)}=1\right\}$ e $\mu_{p}=\inf _{u \in H_{p}}\left(Y_{g}(u)\right)$

Teorema 3.3 Seja $(N, g)$ uma variedade riemanniana compacta de dimensão $n \geq 3$. Dado $p \in$ $\left(2,2^{*}\right)$, então existe uma solução $u=u_{r} \in C^{\infty}(N)$ da equação

$$
\Delta_{g} u+\frac{n-2}{4(n-1)} S_{g} u=\mu_{p} u^{p-1}
$$


considerando que $\int_{N} u^{p} d V_{g}=1$.

\section{Prova:}

Dado $u \in H_{p}$

$$
\begin{aligned}
\left|\int_{N} S_{g} u^{2} d V\right| & \leq \int_{N}\left|S_{g} \| u\right|^{2} d V \\
& \leq\left\|S_{g}\right\|_{L^{\infty}(N)} \int_{N}|u|^{2} d V \\
& \leq\left\|S_{g}\right\|_{L^{\infty}(N)}\|u\|_{L^{p}(N)}^{2}[\lambda(N)]^{2 s} \\
& \leq \kappa .
\end{aligned}
$$

onde $\lambda(N)=\int_{N} d V$ e $s=\frac{1}{2}-\frac{1}{p}$. Decorre que $Y(u)<\infty$ e $\int_{N} S_{g} u^{2} d V \geq-\kappa$, donde

$$
\begin{aligned}
Y(u) & =\int_{N}|\nabla u|^{2} d V+\frac{n-2}{4(n-1)} \int_{N} S_{g} u^{2} d V \\
& \geq \frac{n-2}{4(n-1)} \int_{N} S_{g} u^{2} d V \\
& \geq-\kappa \cdot \frac{n-2}{4(n-1)}
\end{aligned}
$$

concluimos que $\mu$ é finito.

Fato 3.4 Existe $u=u_{p} \in H_{p}, u \geq 0$, tal que $Y(u)=\mu_{p}$.

Considere $u_{k}$ uma sequência minimizante para $\mu_{p}$, isto é, $\left(u_{i} \in H_{p}, \forall k\right.$ e $\left.\lim _{k \rightarrow \infty} Y\left(u_{k}\right)=\mu_{p}\right)$. Observe que $u_{k} \geq 0$, uma vez que $u \in H^{1}(N) \Rightarrow|u| \in H^{1}(N)$ e $|\nabla| u|| \leq|\nabla u|$, além disso

$$
Y\left(u_{k}\right)=\left.\int_{N}|\nabla| u_{k}\right|^{2} d V+\frac{n-2}{4(n-1)} \int_{N} S_{g}\left|u_{k}\right|^{2} d V \leq Y\left(u_{k}\right)
$$

donde $Y\left(\left|U_{k}\right|\right) \longrightarrow \mu_{p}$.

Observe que

$$
\begin{aligned}
\left\|u_{k}\right\|_{H^{1}(N)}^{2}= & \left\|\nabla u_{k}\right\|_{L^{2}(N)}^{2}+\left\|u_{k}\right\|_{L^{2}(N)}^{2} \\
= & \int_{N}\left|\nabla u_{k}\right|^{2} d V+\frac{n-2}{4(n-1)} \int_{N} S_{g} u_{k}^{2} d V+ \\
& -\frac{n-2}{4(n-1)} \int_{N} S_{g} u_{k}^{2} d V+\left\|u_{k}\right\|_{L^{2}(N)}^{2} \\
= & Y\left(u_{k}\right)-\frac{n-2}{4(n-1)} \int_{N} S_{g} u_{k}^{2} d V+\left\|u_{k}\right\|_{L^{2}(N)}^{2} \\
\leq & Y\left(u_{k}\right)+\left(\frac{n-2}{4(n-1)}\left\|S_{g}\right\|_{L^{\infty}(N)}+1\right)\left\|u_{k}\right\|_{L^{2}(N)}^{2} .
\end{aligned}
$$

Para $k \rightarrow \infty$ para que $Y\left(u_{k}\right)=\mu_{p}+1$, usando $\left\|u_{k}\right\|_{L^{2}(N)}^{2} \leq\left\|u_{k}\right\|_{L^{p}(N)}^{2}[\lambda(N)]^{2 s}$, implica para 
alguma constante $\alpha$ que

$$
\mid u_{k} \|_{H^{1}(N)}^{2} \leq\left(\mu_{p}+1\right)+\left(\frac{n-2}{4(n-1)}\left\|S_{g}\right\|_{L^{\infty}(N)}+1\right) \alpha
$$

assim sendo $u_{k}$ é limitada.

Sabendo que $u_{k}$ é limitado, considere $u \in H^{1}(N)$ de modo que $u_{k}$ convirja fracamente para $u$, por Rellich-Kondrakov existe subsequência $u_{k}$ (abuso de notação) tal que $u_{k}$ converge para $u$ na norma $L^{p}(N)$.

Agora

$$
\left\|u_{k}\right\| \leq \liminf \left\|u_{k}\right\|_{H^{1}(N)}^{2} \leq \liminf \left(\left\|\nabla u_{k}\right\|_{L^{2}(N)}^{2}+\left\|u_{k}\right\|_{L^{2}(N)}^{2}\right)
$$

segue

$$
\|\nabla u\|_{L^{2}(N)}^{2}+\|u\|_{L^{2}(N)}^{2} \leq \liminf \left\|\nabla u_{k}\right\|_{L^{2}(N)}^{2}+\|u\|_{L^{2}(N)}^{2}
$$

portanto

$$
\|\nabla u\|_{L^{2}(N)}^{2} \leq \liminf \left\|\nabla u_{k}\right\|_{L^{2}(N)}^{2}
$$

Podemos então mostrar que $Y(u)=\mu_{p}$, de fato

$$
\begin{aligned}
\mu_{p} \leq Y(u) & =\int_{N}|\nabla u|^{2} d V+\frac{n-2}{4(n-1)} \int_{N} S_{g}|u|^{2} d V \\
& \leq \liminf \|\left.\nabla u_{k}\right|_{L^{2}(N)} ^{2}+\frac{n-2}{4(n-1)} \lim _{k \rightarrow \infty} \int_{N} S_{g}\left|u_{k}\right|^{2} d V \\
& =\liminf \left(\int_{N}\left|\nabla u_{k}\right|^{2} d V+\frac{n-2}{4(n-1)} \int_{N} S_{g}\left|u_{k}\right|^{2} d V\right) \\
& =\liminf Y(u)=\mu_{p} .
\end{aligned}
$$

Pelo teorema dos multiplicadores de Lagrange, existe $\rho \in \mathbb{R}$ tal que

$$
\int_{N}\langle\nabla u, \nabla \xi\rangle d V+\frac{n-2}{4(n-1)} \int_{N} S_{g} u \xi d V=\rho \int_{N} u^{p-1} \xi d V
$$

fazendo $\xi=u$, teremos $Y(u)=\rho$ e $\rho=\mu_{p}$ donde concluimos que $u$ é solução fraca para 3.3, para concluirmos que $u$ é positiva devemos aplicar o Princípio do Máximo apoś verificação das hipóteses. 


\section{Capítulo 4}

\section{Número mínimo de soluções para Problema de Yamabe-subcrítico}

\subsection{Centro de Massa Riemaniana}

Agora falaremos um pouco sobre a teoria desenvolvida por H. Karcher e K. Grove em [13] e [11. Seja $A$ um espaço de medida de volume 1 (frequentemente será uma variedade riemaniana compacta ou um conjunto finito de pontos). Seja $M$ uma variedade riemaniana completa e $B(m, r)$ um bola fortemente convexa aberta de raio $r$ centrada em $m$ em $M$. Chamamos qualquer função mensurável $f: A \longrightarrow B(m, r)$ uma distribuição de massa em $B(m, r)$.

$$
P_{f}: \bar{B}_{r} \longrightarrow \mathbb{R}, \quad P_{f}(m)=\frac{1}{2} \int_{A} d(m, f(a)) d a
$$

Teorema $4.1 \operatorname{grad}_{f}(m)=-\int_{A} \exp _{m}^{-1} f(a) d a$. Quando $m \in \partial B(m, r)$, gradP $P_{f(m)}$ é uma média sobre os vetores apontando para fora. $P_{f}$ tem somente mínimo na bola compacta $\overline{B(m, r)}$.

Se a curvatura seccional de $M$ em $B(m, r)$ é no máximo 0 , então ao longo de qualquer geodésica $\gamma: I \rightarrow B(m, r)$

$$
\frac{d^{2}}{d t^{2}} P_{f}(\gamma(t)) \geq\langle\dot{\gamma}, \dot{\gamma}\rangle
$$

Se a curvatura seccional de $M$ em $B(m, r)$ é no máximo $\Delta(>0)$, então $\left(\operatorname{com} \mathcal{S}_{\Delta}(t)=\right.$ $\Delta^{-1 / 2} \sin \left(\Delta^{1 / 2} t\right)$ ao longo de qualquer geodésica $\gamma: I \rightarrow B(m, r)$

$$
\frac{d^{2}}{d t^{2}} P_{f}(\gamma(t)) \geq 2 f \cdot \frac{\mathcal{S}^{\prime} \Delta}{\mathcal{S} \Delta}(2 f)\langle\dot{\gamma}, \dot{\gamma}\rangle
$$

Assuma $2 f<\frac{1}{2} \pi \Delta^{1 / 2}$. Neste caso (1.2.2) e (1.2.3) declara que $P_{f}$ é função convexa de $B(m, r)$.

Prova:

Seja $\gamma: I \rightarrow B(m, r)$ uma geodésica e considere uma família

$$
\left\{c_{a}(s, t)=\exp _{f(a)}\left(s \cdot \exp _{f(a)}^{-1} \gamma(t)\right)\right\}
$$

de geodésicas de $f(a)$ para $\gamma(t)$. Denote 
38CAPÍTULO 4. NÚMERO MÍNIMO DE SOLUÇÕES PARA PROBLEMA DE YAMABE-SUBCRÍTICO

$$
c_{a}^{\prime}=\frac{d}{d s} c_{a}(s, t) \quad \dot{c}_{a}=\frac{d}{d t} c_{a}(s, t)
$$

Sabemos que $d(f(a), \gamma(a))=\left|c_{a}^{\prime}(s, t)\right|$ independe de $s$ e que $s \mapsto \dot{c}_{a}(s, t)$ é uma família de campos de Jacobi. Considere as seguinte identidades $\frac{D}{d t} c^{\prime}=\frac{D}{d s} \dot{c}, \frac{D}{d s} c^{\prime}=0,\left|c^{\prime}\right|$ independe de $s$, $\frac{d}{d s}\left\langle\dot{c}, c^{\prime}\right\rangle=\left\langle\frac{D}{d s} \dot{c}, c^{\prime}\right\rangle,\left(\frac{d}{d t}\right) c_{a}(0,1)=0,\left.\frac{d}{d t}\left(\exp _{f(a)}\left(s \cdot \exp _{f(a)}^{-1}(\gamma(t))\right)\right)\right|_{0}=d\left(\exp _{f(a)}\right)_{0}(0)=0$.

$$
\begin{aligned}
\frac{d}{d t} P_{f}(\gamma(t)) & =\frac{1}{2} \frac{d}{d t} \int_{A}\left\langle c_{a}^{\prime}(s, t), c_{a}^{\prime}(s, t)\right\rangle d a \\
& =\frac{1}{2} \int_{A} \frac{d}{d t}\left\langle c_{a}^{\prime}(S, t), c_{a}^{\prime}(S, t) d a\right. \\
& =\frac{1}{2} \int_{A}\left\langle\frac{D}{d t} c_{a}^{\prime}(S, t), c_{a}^{\prime}(S, t)\right\rangle+\left\langle c_{a}^{\prime}(S, t), \frac{D}{d t} c_{a}^{\prime}(S, t)\right\rangle d a \\
& =\frac{1}{2} \int_{A} 2\left\langle\frac{D}{d t} c_{a}^{\prime}, c_{a}^{\prime}\right\rangle d a=\int\left\langle\frac{D}{d t} c_{a}^{\prime}, c_{a}^{\prime}\right\rangle d a \\
& =\int_{A}\left\langle\frac{D}{d S} \dot{c}_{a}, c_{a}^{\prime}\right\rangle d a \\
& =\int_{A}\left(\int_{0}^{1}\left\langle\frac{D}{d S} \dot{c}_{a}, c_{a}^{\prime}\right\rangle d s\right) d a \\
& =\int_{A} \int_{0}^{1} \frac{D}{d S}\left\langle\dot{c}_{a}, c_{a}^{\prime}\right\rangle d a \\
& =\left.\int_{A}\left\langle\dot{c}_{a}, c_{a}^{\prime}\right\rangle\right|_{0} ^{1} d a=\int\left\langle\dot{c}_{a}(1, t), c_{a}^{\prime}(1, t)\right\rangle-\left\langle\dot{c}_{a}(0, t), c_{a}^{\prime}(1, t)\right\rangle d a \\
& =\int_{A}\left\langle\dot{c}_{a}(1, t), c_{a}^{\prime}(1, t)\right\rangle d a
\end{aligned}
$$




$$
\begin{aligned}
\dot{c}_{a}(1, t) & =\frac{d}{d t} c_{a}(1, t) \\
& =\frac{d}{d t}\left(\exp _{f(a)}\left(1 \cdot \exp _{f(a)}^{-1}(\gamma(t))\right)\right) \\
& =\frac{d}{d t}(\gamma(t))=\dot{\gamma}(t) \\
c_{a}^{\prime}(1, t) & =\frac{d}{d s} c(1, t) \\
& =\frac{d}{d s}\left(\left.\exp _{f(a)}\left(s \cdot \exp _{f(a)}^{-1}(\gamma(t))\right)\right|_{s=1}\right. \\
& =d_{s}\left(\exp _{f(a)}\left(s \cdot \exp _{f(a)}^{-1}(\gamma(t))\right)\right) \cdot \exp _{f(a)}^{-1}(\gamma(t)) \\
& =d\left(\exp _{f(a)}\right)\left(\exp _{f(a)}^{-1}(\gamma(t)) \exp _{f(a)}^{-1}(\gamma(t)\right. \\
& =-\exp _{\gamma(t)}^{-1}(f(a))
\end{aligned}
$$

Decorre que:

$$
\begin{gathered}
\frac{d}{d t} P_{f}(\gamma(t))=\int_{A}\left\langle\dot{c}_{a}(1, t), c_{a}^{\prime}(1, t)\right\rangle d a \\
\frac{d}{d t} P_{f}(\gamma(t))=\int_{A}\left\langle\dot{\gamma}(t),-\exp _{\gamma(t)}(f(a))\right\rangle d a \\
\text { então } \operatorname{grad}\left(P_{f}(\gamma(t))\right)=-\int_{A} \exp _{\gamma(t)}(f(a)) d a .
\end{gathered}
$$

Observe que $\dot{c}(1, t)=\dot{\gamma}(t)$ e $c_{a}^{\prime}(1, t)=-\exp _{\gamma(t)}^{-1}(f(a))$ são vetores tangentes das geodésicas de $f(a)$ para $\gamma(t)$.

$$
\begin{aligned}
\frac{d^{2}}{d t^{2}} P_{f}(\gamma(t)) & =\frac{d}{d t}\left(\frac{d}{d t} P_{f}(\gamma(t))\right) \\
& =\frac{d}{d t}\left(\int_{A}\left\langle\dot{c}_{a}(1, t), c_{a}^{\prime}(1, t)\right\rangle d a\right) \\
& =\int_{A}\left\langle\frac{d}{d t} \dot{c}_{a}(1, t), c_{a}^{\prime}(1, t)\right\rangle d a+\int_{A}\left\langle\dot{c}_{a}(1, t), \frac{d}{d t} c_{a}^{\prime}(1, t)\right\rangle d a \\
& =\int_{A}\left\langle\dot{c}_{a}(1, t), \frac{D}{d s} c_{a}^{\prime}(1, t)\right\rangle d a
\end{aligned}
$$

ou $\frac{D}{d t}(\operatorname{grad})\left(P_{f}(\gamma(t))\right)=\int_{A} \frac{D}{d t} \frac{d}{d t} c_{a}(1, t) d a$.

Nós denotamos o campo de Jacobi $\dot{c}_{a}$ ao longo de $s \mapsto c_{a}(s, t)$ por $J_{a}(s)=c_{a}(s, t)$.

$J(0)=0, J(1)=\gamma(t)$, independe de $a$, e $\left(\frac{D}{d s} \dot{c}_{a}(1, t)\right)=J^{\prime}(1)$.

Para a componente normal $\left(1, \dot{c}_{a}\right)$ deste campo de Jacobi, nós temos a cota inferior [13](A5.1) ao longo de geodésicas de comprimento menor ou igual a $2 r$. 


$$
\left\langle J^{\prime}(1)^{\text {norm }}, J(1)\right\rangle \geq\left\langle J(1)^{\text {norm }}, J(1)\right\rangle \cdot 2 r\left(\frac{S^{\prime} \Delta}{S \Delta}\right)(2 r)
$$

enquanto que para a componente tangencial

$$
J^{\prime}(1)^{\tan }=J(1)^{\tan }
$$

[13] (ver A0.3)

Isto prova as relações (1.2.2) e (1.2.3).

Definição 4.2 (Centro de Massa) Supondo (1.2.2) e (1.2.3), a função convexa $P_{f}$ tem um único mínimo em $B(m, r)$. Chamaremos esse ponto de mínimo de centro de massa $\mathrm{cm}(f)$ de $f$.

\subsubsection{A Função Centro de Massa $c_{m}(r, \eta): L_{r, \eta}^{1}(M) \rightarrow M$}

$(M, g)$ variedade riemanniana fechada, $V \subseteq M$ subconjunto fortemente convexo $(x, y \in V$, existe um único $\gamma$ segmento geodésico normal ligando $x$ a $y$, inteiramente contigo em $V$ ). Uma vez que $M$ é fechado, existe $r_{0}>0$, de modo que para todo $x \in M$ e $r \leq r_{0}$ a bola geodésica de raio $r$ contida em $x, B(x, r)$ é fortemente convexa.

Seja $u \in L^{1}(M)$ não negativa. Considere a função contínua $P_{u}: M \rightarrow \mathbb{R}$, dada por

$$
x \mapsto P_{u}(x)=\int_{M} d(x, y)^{2} u(y) d v_{g}(y)
$$

Se $r$ é suficientemente pequeno e o suporte de $u$ está contido em $B(x, r)$, então H. Karcher e K. Grove em [11] e [13] Definiram o centro de massa riemanniano da função $u$ o qual foi chamado centro de massa da medida dado por $u d v_{g}$, com o único mínimo global da função $P_{u}$, como visto na seção anterior. A função $P_{u}$ é estritamente convexa em uma bola pequena, e o mínimo pode não ser alcançado fora dessa bola. Vamos denotar $L^{1, r}(M)$ espaço de funções $u \in L^{1}(M)$ com suporte em alguma bola geodésica $B(x, r)$. Note que $c_{m}: L^{1, r}(M) \rightarrow M$ dada por $u \mapsto P_{u}$ define uma função contínua $L^{1}(M) \rightarrow C^{0}(M)$ com imagem na família de funções com único mínimo, e o mínimo depende continuamente na $C^{0}$-topologia de tais funções.

J.Petean em [16] esta interessado em estender o conceito de centro de massa Riemanniano para funções que não são suportadas em bolas pequenas. Isto não é possível em geral, pois $P_{u}$ em geral tem mais que um mínimo. Contudo J. Petean dá uma boa definição de centro de massa para funções que se concentram em pequenas bolas, vejamos:

Dado $u \in L^{1}(M)$ e $r>0$,uma $(u, r)$-funções concentração é

$$
C_{u, r}=\frac{\int_{B(x, r)}|u| d v_{g}}{\|u\|_{1}}
$$

Observe que $C_{u, r}: M \rightarrow[0,1]$ é contínua, de fato: se $r \geq \operatorname{diam}(M) \Rightarrow C_{u, r} \equiv 1$ e $\forall x \in M$ temos

$$
\lim _{r \rightarrow 0} C_{u, r}(x)=0 .
$$

O $r$-coeficiente de concentração de $u$ é o maximo de $C_{u, r}$ 


$$
C_{r}(u)=\sup _{x \in M} \frac{\int_{B(x, r)}|u| d v_{g}}{\|u\|_{1}}
$$

$\forall \mu \in(0,1)$, seja $L_{r, \mu}^{1}:=\left\{u \in L^{1}(M) ; c_{r}(u)>\mu\right\}\left(C_{u, r}, C_{r}\right.$ depende de $\left.g\right)$, iremos fixar $g$ e escrever $L_{r, \mu}^{1}(M, g)=L_{r, \mu}^{1}(M)$.

$\forall \eta \in\left(\frac{1}{2}, 1\right)$ considere a função continua e linear $\varphi_{\eta}: \mathbb{R} \rightarrow[0,1]$ dada por

$t \mapsto \varphi_{\eta}(t)=\left\{\begin{array}{l}0 \text { se } t \leq 1-\eta \\ 1 \text { se } t \geq \eta\end{array} \quad\right.$ é linear e crescente em $[1-\eta, \eta]$.

Fixe $r<\left(\frac{1}{2}\right) r_{0}$ e $\forall u \subseteq L_{r, \eta}^{1}(M)$ seja

$$
\Phi_{r, \eta}(u)(x):=\varphi_{\eta}\left(c_{\mu, r}(x)\right) \cdot u(x)
$$

Lema 4.3 $\forall u \in L_{r, \eta}^{1}(M)$ o suporte de $\Phi_{r, \eta}(u)$ está contido em uma bola geodésica de raio $2 r$ (centrada em um ponto de máxima concentração).

Dado $x \in M ; C_{u, r}$ tem um máximo $C_{u, r}(x) \geq \eta$.

Se $d(x, y)>2 r \Rightarrow B(x, r) \cap B(y, r)=\emptyset \Rightarrow C_{u, r}(y) \leq 1-\eta C_{u, r}: M \rightarrow[0,1]$. Portanto

$$
\Phi_{r, \eta}(u)(y)=\underbrace{\varphi_{\eta}\left(C_{u, r}(y)\right)}_{\leq 1-\eta} \cdot u(x)=0
$$

e o suporte de $\Phi_{r, \eta}(u)$ está contido em $B(x, 2 r)$.

Teorema 4.4 Para qualquer $r<\left(\frac{1}{2}\right) r_{0}$ e $\eta>\frac{1}{2}$ existe uma função contínua $c_{m}(r, \eta): L_{r, \eta}^{1}(M) \rightarrow$ $M$ tal que se $x \in M$ verifica $C_{u, r}(x)>\eta$ então $c_{m}(r, \eta)(u) \in B(x, 2 r)$.

Definição $4.5 c_{m}(r, \eta): L_{r, \eta}^{1}(M) \rightarrow M$ pela prescrição $u \mapsto c_{m}(r, \eta)=c m\left(\Phi_{r, \eta}(u)\right)$

$$
\begin{aligned}
c m\left(\Phi_{r, \eta}(u)\right)(x) & =c m\left(\Phi_{r, \eta}(u)(x)\right) \\
& =c m\left(\varphi_{\eta}\left(C_{r, \eta}(x)\right) \cdot u(x)\right) \\
& =c m(u(x)) \\
& =\operatorname{Min} P_{u}(x)=\operatorname{Min} \int_{M}(d(x, y))^{2} \cdot u(y) d v_{g}(y) .
\end{aligned}
$$

Definição 4.6 Qualquer função $c_{m}(r, \eta)(u)$ como no teorema 4.4 será chamada $(r, \eta)$-centro de massa Riemanniano de $u$. 


\subsection{Teorema Principal}

Nessa seção vamos expor os resultado principal da nossa pesquisa,inicialmente prescisamos apresentar alguns resultado necessarios para a demonstação.

Definição 4.7 (Categoria de Lusternik-Schnirelmann): O subconjunto $A$ do espaço topológico $X$ é dito contrátil em $X$ se a inclusão $i: A \hookrightarrow X$ é homotópica a função constante de $A$ para $B . A$ tem (LS)-categoria igual a $k$ em $X(\operatorname{cat}(A, X)=k)$ se $k$ é a menor quantidade de subconjuntos fechados contrateis de $X$ que cobre $A$, considere $\operatorname{cat}(X):=\operatorname{cat}(X, X)$.

Teorema 4.8 Seja $J$ um $C^{1}$-funcional em uma variedade de Banach completa $C^{1,1} M$. Se $J$ é limitado inferiormente e satisfaz (PS)-condição, então ele tem pelo menos cat $\left(J^{d}\right)$ pontos críticos em $J^{d}$, onde $J^{d}:=\{u \in M: J(u)<d\}$. Além disso se $M$ é contrátil e cat $\left(J^{d}\right)>1$ então existe pelo menos um ponto crítico $u \notin J^{d}$.

Teorema 4.9 Sejam $X, Y$ espaços topológicos. Se $f: X \rightarrow Y$ e $g: Y \rightarrow X$ são contínuas e tais que $g \circ f \stackrel{\text { homot }}{\cong} I d_{X}$, então $\operatorname{cat}(X) \leq \operatorname{cat}(Y)$.

Teorema 4.10 Seja $\left(M^{m}, g_{M}\right)$ uma variedade riemanniana compacta, com $m \geq 3$ e $\left(B^{n}, g_{B}\right)$ uma variedade riemanniana. Se $\pi:\left(M^{m}, g_{M}\right) \rightarrow\left(B^{n}, g_{B}\right)$ é uma submersão riemanniana com fibras minimas e de curvatura escalar constante positiva, então, para $\varepsilon>0$ suficientemente pequeno, existem cat $(B)+1$ métricas conformes à $g_{M}^{\varepsilon}=g_{B}+\varepsilon g_{\mathcal{F}}$ com curvatura escalar constante.

Considere $\left(M^{m}, g_{B}+\varepsilon^{2} g_{\mathcal{F}}\right)$, onde ambas as métricas $g_{B}$ e $g_{\mathcal{F}}$ tem curvatura escalar constante, respectivamente $S_{g_{B}}$ e $S_{g_{\mathcal{F}}}$, em nosso contexto uma métricas conformes a $g_{M}^{\varepsilon}$ será

$$
\tilde{g}=u^{\frac{4}{m-2}} \cdot g_{M}^{\varepsilon}
$$

onde $u$ satisfaz a $\left(g_{B}+\varepsilon^{2} g_{\mathcal{F}}\right)$-Equação de Yamabe no caso subcritico,

$$
-a_{m} \varepsilon+\left(S_{g_{B}}+\varepsilon^{-2} S_{g_{\mathcal{F}}}\right) u=u^{p_{m}-1}
$$

para $a_{m}=\frac{4(m-1)}{m-2}$ e $p_{m}=\frac{2 m}{m-2}$ é o expoente crítico de Sobolev. As funções $u: B \rightarrow \mathbb{R}_{>0}$ que satisfazem a equação 4.2 são consideradas como funções de $M$, caso contrário a equação 4.2 deixará de ser subcritica, de fato, por hipótese $m>n$ isso implica que $p_{m}<p_{n}$.

Dado $\varepsilon>0$ suficientemente pequeno de forma que $\left(S_{g_{B}}+\varepsilon^{-2} S_{g_{\mathcal{F}}}\right)$ é positivo, podemos reescrever a equação 4.2 da seguinte forma

$$
-a_{m} \Delta_{g} u+\left(S_{g_{B}}+\varepsilon^{-2} S_{\mathcal{F}}\right) u=\left(\varepsilon^{-2} S_{\mathcal{F}}\right) u^{p_{m}-1}
$$

Normalizando $g_{\mathcal{F}}$ e supondo que $S_{\mathcal{F}}=a_{n}$, podemos reescrever a equação acima da seguinte forma

$$
-\varepsilon^{-2} \Delta_{g} u+\left(\left(S_{g_{B}} / a_{m}\right) \varepsilon^{2}+1\right) u=u^{p_{m}-1}
$$

Seja $J_{\varepsilon}: H^{1}(B) \rightarrow \mathbb{R}$ o $C^{2}$ funcional energia associado a equação anterior, definido definido como

$$
J_{\varepsilon}(u)=\frac{1}{\varepsilon^{m}} \int_{B}\left(\frac{1}{2} \varepsilon^{2}\|\nabla u\|^{2}+\frac{S_{g_{B}} \varepsilon^{-2}+a_{m}}{2 a_{m}} u^{2}-\frac{1}{p_{m}}\left(u^{+}\right)^{p_{m}}\right) d \mu
$$


a variedade de Nehari associada ao funcional $J_{\varepsilon}(u)$ é:

$$
\left.N_{\varepsilon}=\left\{u \in H^{1}(B)\{0\}: \int_{B}\left(\varepsilon^{2}|\nabla u|^{2}+\left(S_{g_{B}} / a_{m}\right) \varepsilon^{2}+1\right) u^{2}\right) d \mu_{g_{B}}=\int_{B}\left(u^{+}\right)^{p_{m}}\right\}
$$

O mínimo de $J_{\varepsilon}$ é definido como

$$
\mathbf{m}_{\varepsilon}=\inf _{u \in N_{\varepsilon}} J_{\varepsilon}(u)=\varepsilon^{-m}\left(1 / 2-1 / p_{m}\right) \inf _{u \in N_{\varepsilon}} \int\left(u^{+}\right)^{p_{m}} d \mu
$$

e $\left.\sum_{\varepsilon, \mathbf{m}_{\varepsilon}+\delta}=\left\{u \in N_{\varepsilon} ; J_{\varepsilon}(u)<\mathbf{m}_{\varepsilon}+\delta\right)\right\}$ é o subnível do funcional $J_{\varepsilon}$. Provar o teorema 4.10 equivale a mostrar que a $\left(g_{B}+\varepsilon g_{\mathcal{F}}\right)$-Equação de Yamabe tem $\operatorname{cat}(B)+1$ soluções, pela teoria de Lusternik-Schnirelmann.

Fato 4.11 Kazuo Akutagawa, Luiz Florit e Jimmy Petean em seu trabaho On Yamabe constants of Riemannian products de 2006 [1] e Jimmy Petean em [16], estudaram para $\varepsilon>0$ o comportamento da constante de Yamabe em uma variedade produto. Aplicando o teorema 1.1 de [1] a variedade produto $\left(N \times M, g+\varepsilon^{2} h\right)$ formada por duas variedades riemanianas fechadas, a saber $\left(N^{m}, h\right)$ de curvatura escalar constante positiva e $\left(M^{n}, g\right)$. Podemos restringir o $\left(g+\varepsilon^{2} h\right)$-funcional de Yamabe as funções que dependam somente de $(M, g)$. Seja

$$
Y_{M}\left(N \times M, \varepsilon^{2} h+g\right)=\inf _{u \in H^{1}(M)-\{0\}}\left(Y_{\varepsilon^{2} h+g}(u)\right)
$$

a constante de Yamabe, para a métrica euclidiana $g_{E}$ em $\mathbb{R}^{n}$, Jimmy Petean em [16] mostra que

$$
\lim _{\varepsilon \rightarrow 0} Y_{M}\left(N \times M, \varepsilon^{2} h+g\right)=Y_{\mathbb{R}^{n}}\left(N \times \mathbb{R}^{n}, h+g_{E}\right)
$$

para $V=\operatorname{Vol}(N, h)$ então $V^{\frac{-2}{m}} h$ tem volume 1 e $S_{V^{\frac{-2}{n}} h}=V^{\frac{2}{m}} \mathbf{a}$, onde $\mathbf{a}=\frac{4((m+n)-1)}{(m+n)-2}, \mathbf{p}=\frac{2(m+n)}{(m+n)-2}$ o expoente critico de Sobolev. Decorre por [1] teorema 1.4 que:

$$
Y_{\mathbb{R}^{n}}\left(N \times \mathbb{R}^{n}, h+g_{E}\right)=\mathbf{a} V^{\frac{2}{(m+n)}}((m+n)(\tilde{\mathbf{m}}(E)))^{\frac{2}{m+n}}
$$

onde $\tilde{\mathbf{m}}(E)=\frac{\mathbf{p}-2}{2 \mathbf{p}}\|U\|_{\mathbf{p}}^{\mathbf{p}-2}$.

Alem disso quando $u \in H^{1}(M)-0$,

$$
Y_{\varepsilon^{2} h+g}(u)=\operatorname{Vol}\left(N, \varepsilon^{2} h\right)^{1-\frac{2}{p}} \frac{\int_{M} \mathbf{a}\|\nabla u\|^{2}+\left(\varepsilon^{2} S_{h}+S_{g}\right) u^{2} d v_{g}}{\|u\|_{p}^{2}}
$$

então segue do teorema 4.11 que:

$$
Y_{\mathbb{R}^{n}}\left(N \times \mathbb{R}^{n}, h+g_{E}\right)=\operatorname{Vol}_{\varepsilon \rightarrow 0}\left(N, \varepsilon^{2} h\right)^{\frac{2}{m+n}} \inf _{u \in H^{1}(M)} \frac{\mathbf{a}}{\varepsilon^{2}} \frac{\int_{M} \mathbf{a}\|\nabla u\|^{2}+\left(1+\varepsilon^{2} S_{g} / \mathbf{a}\right) u^{2} d v_{g}}{\|u\|_{p}^{2}}
$$

Como o ínfimo do lado direito é atingido por funções positivas e o quociente é invariante por hometetias, podemos tomar o infimo sobre a variedade de Nehari correspondente, portanto:

$$
Y_{\mathbb{R}^{n}}\left(N \times \mathbb{R}^{n}, h+g_{E}\right)=\lim _{\varepsilon \rightarrow 0} \mathbf{a} \varepsilon^{\frac{-2 n}{m+n}} V^{\frac{2}{m+n}} \inf _{u \in N_{\varepsilon}}\left(\int_{M} u^{p}\right)^{\frac{p-2}{p}}=\mathbf{a} V^{\frac{2}{(m+n)}}\left((m+n)\left(\lim _{\varepsilon \rightarrow 0} \mathbf{m}_{\varepsilon}\right)\right)^{\frac{2}{m+n}}
$$


implica que

$$
\lim _{\varepsilon \rightarrow 0} \mathbf{m}_{\varepsilon}=\mathbf{m}(E)
$$

de modo análogo a 4.11 , ocorre com os funcinais $J_{\varepsilon} 4.3$ e $E 2.12$

\subsubsection{Construção de uma função radial $\phi_{\varepsilon}$}

Defina $\varphi_{r}: \mathbb{R}_{+} \rightarrow[0,1]$

$$
\varphi_{r}(t):=\left\{\begin{array}{l}
1 \text { se } 0 \leq t \leq \frac{r}{2} \\
0 \text { se } r \leq t
\end{array}\right.
$$

suave e $\left|\varphi_{r}^{\prime}(t)\right| \leq \frac{2}{r}$, onde $r$ é definido na seção 1.5 . Fixado $q \in B$ e $\varepsilon>0$. Vamos definir em $B$ a seguinte função

$$
\mathcal{W}_{q, \varepsilon}(x):=\left\{\begin{array}{l}
U_{\varepsilon}\left(\exp _{q}^{-1}(x)\right) \varphi_{r}\left(\left|\exp _{q}^{-1}(x)\right|\right) \text { se } x \in B_{g}(q, r) \\
0, \text { caso contrário. }
\end{array}\right.
$$

$U_{\varepsilon}(z)=U(z / \varepsilon) 2.4$ é uma função positiva, esfericamente simétrica. Consequentemente $\mathcal{W}_{q, \varepsilon}^{+}(x)=$ $W_{q, \varepsilon}(x)$. Alem disso o suporte de $\mathcal{W}_{q, \varepsilon}: B \rightarrow \mathbb{R}$ esta contido em $B(q, r)$.

Fato 4.12 Para qualquer função $u \in H_{g}^{1}(B), u^{+} \not \equiv 0\left(\int_{M} u^{+}>0\right)$, existe uma única $\lambda(u) \in \mathbb{R}_{+}$, tal que $\lambda(u) u \in N_{\varepsilon}$ onde

$$
\lambda^{p_{m}-2}(u)=\frac{\int_{B} \varepsilon^{2}\left|\nabla_{g} u(x)\right|^{2}+\left(\varepsilon^{2}\left(S_{g} / a_{m}\right)+1\right) u(x)^{2} d \mu_{g}}{\int_{B}\left(u(x)^{+}\right)^{p_{m}} d \mu_{g}}
$$

A função $\mathcal{W}_{q, \varepsilon} \in H_{g}^{1}(B)$.

Definição 4.13

$$
\begin{gathered}
\phi_{\varepsilon}: B \rightarrow N_{\varepsilon} \\
x \mapsto \phi_{\varepsilon}(x):=\lambda\left(\mathcal{W}_{q, \varepsilon}\right) \mathcal{W}_{q, \varepsilon}
\end{gathered}
$$

Teorema 4.14 Para todo $\varepsilon>0$, a função $\phi_{\varepsilon}: B \rightarrow N_{\varepsilon}$ é contínua. Além disso para algum $\delta>0$ existe $\varepsilon_{0}>0$ tal que se $\varepsilon<\varepsilon_{0}$ então $\phi_{\varepsilon}(q) \in \sum_{\varepsilon, m(E)+\delta}$ para todo $q \in M$.

\section{Prova:}

A prova desse teorema é baseada na proposição 4.2 da referencia 3 . Fixado $\varepsilon>0$, quando consideramos $\lambda(u)$ como uma função de $u \in H^{1}(B)$, temos que $\lambda(u)$ é contínua, logo basta mostrar que $\mathcal{W}_{q, \varepsilon}$ é continua, i.e,

$$
\lim _{k \rightarrow \infty}\left\|\mathcal{W}_{q_{k}, \varepsilon}-\mathcal{W}_{q, \varepsilon}\right\|=0 \text { e } \lim _{k \rightarrow \infty}\left\|\nabla \mathcal{W}_{q_{k}, \varepsilon}-\nabla \mathcal{W}_{q, \varepsilon}\right\|=0
$$

para qualquer sequência $\left(q_{k}\right) \subset B$ tal que $q_{k} \rightarrow q$ quando $k \rightarrow \infty$. Como o suporte de $\mathcal{W}_{q, \varepsilon}$ 
está contido na bola $B(q, r)$, temos

$$
\int_{B}\left(\mathcal{W}_{q_{k}, \varepsilon}-\mathcal{W}_{q, \varepsilon}\right)^{2} d \mu=\int_{B(q, r)}\left(\mathcal{W}_{q_{k}, \varepsilon}-\mathcal{W}_{q, \varepsilon}\right)^{2} d \mu
$$

Considere o conjunto

$$
Z_{k}=\left[B\left(q_{k}, r\right) \backslash B(q, r)\right] \cup\left[B(q, r) \backslash B\left(q_{k}, r\right)\right]
$$

Quando $k \rightarrow \infty$ temos $q_{k} \rightarrow q$, consequentemente teremos $\mu\left(Z_{k}\right) \rightarrow 0$. então

$$
\int_{Z_{k}}\left(\mathcal{W}_{q_{k}, \varepsilon}-\mathcal{W}_{q, \varepsilon}\right)^{2} d \mu \rightarrow 0
$$

quando $k \rightarrow \infty$.

Agora vamos considerar $\eta_{k}(z):=\exp _{q_{k}}^{-1}\left(\exp _{q}(z)\right)$ e $A_{k}:=\exp _{q}^{-1}\left(B(q, r) \cap B\left(q_{k}, r\right)\right)$, observe que para $k \rightarrow \infty$ temos $\eta_{k}(z) \rightarrow z$ e $A_{k} \rightarrow B(0, r)$, ou seja, $Z_{k} \cup \exp _{q}\left(A_{k}\right) \rightarrow B(q, r)$, podemos reescrever a integral da sequinte forma

$$
\int_{B\left(q_{k}, r\right)}\left(\mathcal{W}_{q_{k}, \varepsilon}-\mathcal{W}_{q, \varepsilon}\right)^{2} d \mu \rightarrow \int_{Z_{k}}\left(\mathcal{W}_{q_{k}, \varepsilon}-\mathcal{W}_{q, \varepsilon}\right)^{2} d \mu+\int_{\exp _{q}\left(A_{k}\right)}\left(\mathcal{W}_{q_{k}, \varepsilon}-\mathcal{W}_{q, \varepsilon}\right)^{2} d \mu
$$

é suficiente mostrar que

$$
\int_{\exp _{q}\left(A_{k}\right)}\left(\mathcal{W}_{q_{k}, \varepsilon}(x)-\mathcal{W}_{q, \varepsilon}(x)\right)^{2} d \mu \rightarrow 0
$$

para $k \rightarrow \infty$.

Observe que

$\eta_{k}(z)=\exp _{q_{k}}^{-1}\left(\exp _{q}(z)\right) \Rightarrow \eta_{k}\left(\exp _{q(x)}^{-1}\right)=\exp _{q_{k}}^{-1}\left(\exp _{q}\left(\exp _{q}^{-1}(x)\right)=\exp _{q_{k}}^{-1}(x)\right.$

e $x=\exp _{q_{k}}\left(\eta_{k}\left(\exp _{q}^{-1}(x)\right)\right)$

Dai

$$
\begin{aligned}
& \int_{\exp _{q}\left(A_{k}\right)}\left(\mathcal{W}_{q_{k}, \varepsilon}(x)-\mathcal{W}_{q, \varepsilon}(x)\right)^{2} d \mu \\
& =\int_{A_{k}}\left(\mathcal{W}_{q_{k}, \varepsilon}\left(\exp _{q_{k}}\left(\eta_{k}\left(\exp _{q}^{-1}(x)\right)\right)\right)-\mathcal{W}_{q, \varepsilon}(x)\right)^{2} d \mu \\
& =\int_{A_{k}}\left(U _ { \varepsilon } ( \operatorname { e x p } _ { q _ { k } } ^ { - 1 } ( \operatorname { e x p } _ { q _ { k } } ( \eta _ { k } ( \operatorname { e x p } _ { q } ^ { - 1 } ( x ) ) ) ) ) \cdot \varphi _ { r } \left(\mid \exp _{q_{k}}^{-1}\left(\exp _{q_{k}}\left(\eta_{k}\left(\exp _{q}^{-1}(x)\right)\right) \mid\right)\right.\right. \\
& \left.-U_{\varepsilon}\left(\exp _{q}^{-1}(x)\right) \varphi_{r}\left(\left|\exp _{q}^{-1}(x)\right|\right)\right)^{2} d \mu \\
& =\int_{A_{k}}\left(U_{\varepsilon}\left(\eta_{k}(z)\right) \cdot \varphi_{r}\left(\eta_{k}(z)\right)-U_{\varepsilon}(z) \cdot \varphi_{r}(z)\right)^{2}\left|g_{q}(z)\right|^{\frac{1}{2}} d z \\
& \leq^{i} 2 \int_{A_{k}}\left(U_{\varepsilon}\left(\eta_{k}(z)\right)\left|\varphi_{r}\left(\eta_{k}(z)\right)-\varphi_{r}(z)\right|\right)^{2}\left|g_{q}(z)\right|^{\frac{1}{2}} d z+2 \int_{A_{k}}\left(\varphi_{r}(z)\left|U_{\varepsilon}\left(\eta_{k}(z)\right)-U_{\varepsilon}(z)\right|\right)^{2}\left|g_{q}(z)\right|^{\frac{1}{2}} d z \\
& \leq^{i i} K \int_{A_{k}}\left|\eta_{k}(z)-z\right|\left|g_{q}(z)\right|^{\frac{1}{2}} d z \rightarrow 0 \text { quando } k \rightarrow \infty .
\end{aligned}
$$

Decorre do cálculo acima que $\mathcal{W}_{q, \varepsilon}(x)$ é continua. 


\section{CAPÍTULO 4. NÚMERO MÍNIMO DE SOLUÇÕES PARA PROBLEMA DE YAMABE-SUBCRÍTICO}

A desigualdade $(i)$ é justificada pelo seguinte fato:

$$
\begin{aligned}
& \left(U_{\varepsilon}\left(\eta_{k}(z)\right) \cdot \varphi_{r}\left(\eta_{k}(z)\right)-U_{\varepsilon}(z) \cdot \varphi_{r}(z)\right)^{2} \\
& =\left(U_{\varepsilon}\left(\eta_{k}(z)\right) \cdot \varphi_{r}\left(\eta_{k}(z)\right)-U_{\varepsilon}\left(\eta_{k}(z)\right) \cdot \varphi_{r}(z)+U_{\varepsilon}\left(\eta_{k}(z)\right) \cdot \varphi_{r}(z)-U_{\varepsilon}(z) \cdot \varphi_{r}(z)\right)^{2} \\
& =\left(U_{\varepsilon}\left(\eta_{k}(z)\right)\left(\varphi_{r}\left(\eta_{k}(z)\right)-\varphi_{r}(z)\right)+\varphi_{r}(z)\left(U_{\varepsilon}\left(\eta_{k}(z)\right)-U_{\varepsilon}(z)\right)\right)^{2} \\
& \leq 2\left(U_{\varepsilon}\left(\eta_{k}(z)\right)\left|\varphi_{r}\left(\eta_{k}(z)\right)-\varphi_{r}(z)\right|\right)^{2}+2\left(\varphi_{r}(z)\left|U_{\varepsilon}\left(\eta_{k}(z)\right)-U_{\varepsilon}(z)\right|\right)^{2}
\end{aligned}
$$

Na linha acima usamos a seguinte relação; $\|u+v\|^{2} \leq 2\|u\|^{2}+2\|v\|^{2}$

A desigualdade (ii) é uma aplicação do teorema do valor médio e a limitação das funções $U_{\varepsilon}(z)$ e $\varphi_{r}(z)$, dessa forma aparece a constante $K=K(\varepsilon, r)$, Analogamente teremos

$$
\lim _{k \rightarrow \infty}\left\|\nabla \mathcal{W}_{q_{k}, \varepsilon}-\nabla \mathcal{W}_{q, \varepsilon}\right\|=0
$$

A segunda parte do teorema é mostrar que $\phi_{\varepsilon}(q) \in \sum_{\varepsilon, \mathrm{m}(E)+\delta}$, ou seja, $J_{\varepsilon}\left(\phi_{\varepsilon}(q)\right)<\mathrm{m}(E)+\delta$, para tanto vamos mostrar inicialmente que os três limites abaixo valem unifomente com respeito a $q \in B$

$$
\begin{gathered}
\lim _{\varepsilon \rightarrow 0} \frac{1}{\varepsilon^{n}}\left|\mathcal{W}_{q, \varepsilon}\right|_{2}^{2}=|U|_{2}^{2}, \\
\lim _{\varepsilon \rightarrow 0} \frac{\varepsilon^{2}}{\varepsilon^{n}}\left|\nabla \mathcal{W}_{q, \varepsilon}\right|_{2}^{2}=|\nabla U|_{2}^{2}, \\
\lim _{\varepsilon \rightarrow 0} \frac{1}{\varepsilon^{n}}\left|\mathcal{W}_{q, \varepsilon}\right|_{p_{m}}^{p_{m}}=|U|_{p_{m}}^{p_{m}}
\end{gathered}
$$

Fixado $q \in B$, por definição $\mathcal{W}_{q, \varepsilon} \in H^{1}(B)$, temos

$$
\frac{1}{\varepsilon^{n}} \int_{B}\left|\mathcal{W}_{q, \varepsilon}\right|^{2} d \mu=\frac{1}{\varepsilon^{n}} \int_{B(0, r)} U_{\varepsilon}^{2}(z) \cdot \varphi_{r}^{2}(|z|) \cdot\left|g_{q}(z)\right|^{\frac{1}{2}} d z
$$

Vamos estimar a seguinte diferença:

$$
\begin{aligned}
& \left.\left|\frac{1}{\varepsilon^{n}}\right| \mathcal{W}_{q, \varepsilon}\right|_{2} ^{2}-|U|_{2}^{2} \mid \\
& =\left.\left|\frac{1}{\varepsilon^{n}} \int_{B(0, r)} U_{\varepsilon}^{2}(z) \cdot \varphi_{r}^{2}(|z|) \cdot\right| g_{q}(z)\right|^{\frac{1}{2}} d z-\int_{\mathbb{R}^{n}} U^{2} d z \mid \\
& =\left.\left|\int_{\mathbb{R}^{n}} U^{2}(w) \cdot \varphi_{\frac{r}{\varepsilon}}^{2}(|w|) \cdot\right| g_{q}(\varepsilon w)\right|^{\frac{1}{2}} d w-\int_{\mathbb{R}^{n}} U^{2} d w \mid\left[w:=\frac{z}{\varepsilon} \Rightarrow z=\varepsilon w \mathrm{e} d w=\frac{d z}{\varepsilon}\right] \\
& =\left|\int_{\mathbb{R}^{n}} U^{2}(w)\left(\varphi_{\frac{r}{\varepsilon}}^{2}(|w|) \cdot\left|g_{q}(\varepsilon w)\right|^{\frac{1}{2}}-1\right) d w\right| \\
& \leq\left.\int_{\mathbb{R}^{n}} U^{2}(w)\left|\varphi_{\frac{r}{\varepsilon}}^{2}(|w|) \cdot\right| g_{q}(\varepsilon w)\right|^{\frac{1}{2}}-1 \mid d w \\
& =\left.\int_{B(0, s)} U^{2}(w)\left|\varphi_{\frac{r}{\varepsilon}}^{2}(|w|) \cdot\right| g_{q}(\varepsilon w)\right|^{\frac{1}{2}}-1\left|d w+\int_{\mathbb{R}^{n} \backslash B(0, s)} U^{2}(w)\right| \varphi_{\frac{r}{\varepsilon}}^{2}(|w|) \cdot\left|g_{q}(\varepsilon w)\right|^{\frac{1}{2}}-1 \mid d w
\end{aligned}
$$


Quando $s \rightarrow \infty$ implica que

$$
\left.\int_{\mathbb{R}^{n} \backslash B(0, s)} U^{2}(w)\left|\varphi_{\frac{r}{\varepsilon}}^{2}(|w|) \cdot\right| g_{q}(\varepsilon w)\right|^{\frac{1}{2}}-1 \mid d w \rightarrow 0
$$

Fixado $s>0$ suficientemente grande, pela regularidade da exp vale que $|z|<s$, decorre que

$$
\left.\lim _{\varepsilon \rightarrow 0}\left|\varphi_{\frac{r}{\varepsilon}}^{2}(|w|) \cdot\right| g_{q}(\varepsilon w)\right|^{\frac{1}{2}}-1 \mid=0
$$

uniformemente com relação a $q \in B$ e $z \in B(0, s)$, de fato, $\left|\varphi_{\frac{r}{\varepsilon}}^{2}(|w|)\right| \leq 1$ e $\left|g_{q}(0)\right| \leq 1$. Portanto temos a prova da sentença (4.4), a sentença (4.6) decorre de modo análoga.

Vamos provar o limite (4.5) usando os argumentos anteriores

$$
\begin{aligned}
& \left.\left|\frac{\varepsilon^{2}}{\varepsilon^{n}}\right| \nabla \mathcal{W}_{q, \varepsilon}\right|_{2} ^{2}-|\nabla U|_{2}^{2} \mid \\
& =\left.\left|\int_{B(0, r)} \sum_{i, j=1}^{n} g_{q}^{i j}(z) \frac{\partial U_{\varepsilon}(z) \varphi_{r}(|z|)}{\partial z_{i}} \frac{\partial U_{\varepsilon}(z) \varphi_{r}(|z|)}{\partial z_{j}}\right| g_{q}(z)\right|^{1 / 2} d z-\int_{\mathbb{R}^{n}} \sum_{i, j=1}^{n} g_{q}^{i j} \frac{\partial U}{\partial z_{i}} \frac{\partial U}{\partial z_{j}}\left|g_{q}\right|^{1 / 2} d z \mid \\
& \leq\left.\left|\int_{\mathbb{R}^{n}} \sum_{i, j=1}^{n} g_{q}^{i j}(\varepsilon w) \frac{\partial U(w) \varphi_{\frac{r}{\varepsilon}}(|w|)}{\partial w_{i}} \frac{\partial U(w) \varphi_{\frac{r}{\varepsilon}}(|w|)}{\partial w_{j}}\right| g_{q}(\varepsilon w)\right|^{1 / 2} d w-\int_{\mathbb{R}^{n}} \sum_{i, j=1}^{n} g_{q}^{i j} \frac{\partial U}{\partial w_{i}} \frac{\partial U}{\partial w_{j}}\left|g_{q}\right|^{1 / 2} d w \mid \\
& \leq\left.\left|\int_{\mathbb{R}^{n}} \sum_{i, j=1}^{n} g_{q}^{i j}(\varepsilon w)\left(\frac{\partial U(w) \varphi_{\frac{r}{\varepsilon}}(|w|)}{\partial w_{i}} \frac{\partial U(w) \varphi_{\frac{r}{\varepsilon}}(|w|)}{\partial w_{j}}-\frac{\partial U(w)}{\partial w_{i}} \frac{\partial U(w)}{\partial w_{j}}\right)\right| g_{q}(\varepsilon w)\right|^{1 / 2} d w \mid \\
& \leq\left.{ }^{\left(I_{1}\right)} \int_{\mathbb{R}^{n}}\left|\sum_{i, j=1}^{n} \frac{\partial U(w)}{\partial w_{i}} \frac{\partial U(w)}{\partial w_{j}}\right| \varphi_{\frac{r}{\varepsilon}}(|w|) g_{q}^{i j}(\varepsilon w)|| g_{q}(\varepsilon w)\right|^{1 / 2}-\delta_{i j}|| d w \\
& +{ }^{\left(I_{2}\right)} \int_{\mathbb{R}^{n}}\left|\sum_{i, j=1}^{n} g_{q}^{i j}(\varepsilon w)\right| U(w) \varphi_{\frac{r}{\varepsilon}}(|w|)\left(\frac{\partial U(w)}{\partial w_{i}} \frac{\partial \varphi_{\frac{r}{\varepsilon}}(|w|)}{\partial w_{j}}+\frac{\partial U(w)}{\partial w_{j}} \frac{\partial \varphi_{\frac{r}{\varepsilon}}(|w|)}{\partial w_{i}}\right)||\left|g_{q}(\varepsilon w)\right|^{1 / 2} d w \\
& +{ }^{\left(I_{3}\right)} \int_{\mathbb{R}^{n}}\left|\sum_{i, j=1}^{n} g_{q}^{i j}(\varepsilon w)\right| U^{2}(w) \frac{\partial \varphi_{\frac{r}{\varepsilon}}(|w|)}{\partial w_{i}} \frac{\partial \varphi_{\frac{r}{\varepsilon}}(|w|)}{\partial w_{j}}||\left|g_{q}(\varepsilon w)\right|^{1 / 2} d w
\end{aligned}
$$

considere as integrais $I_{1}, I_{2}$ e $I_{3}$, pela definição da função $\varphi_{r}(z)$, temos que $\left|\varphi_{r}(|z|)\right| \leq 1$ e $\left|\varphi_{r}^{\prime}(|z|)\right| \leq$ $\frac{2}{r}$, nós temos que existe uma constante $\mathcal{C}>0$ dependendo somente da variedade $B$ tal que;

$$
I_{2}+I_{3} \leq \frac{4 n \mathcal{C} \varepsilon}{r} \int_{\mathbb{R}^{n}}\left(U(w)|\nabla U(w)|+\frac{\varepsilon}{r} U^{2}(z)\right) d z
$$

$\operatorname{logo} I_{2}+I_{3} \rightarrow 0$ uniformemente em $q \in B$ quando $\varepsilon \rightarrow 0$, pela limitação de $U$, a integral $I_{1}$ converge para 0 de maneira analoga a aos limites (4.4) e (4.6). Como $\phi_{\varepsilon}(q) \in N_{\varepsilon}$, temos

$$
J_{\varepsilon}\left(\phi_{\varepsilon}(q)\right)=J_{\varepsilon}\left(\lambda\left(\mathcal{W}_{q, \varepsilon}\right) \mathcal{W}_{q, \varepsilon}\right)=\frac{1}{\varepsilon^{m}}\left(\frac{1}{2}-\frac{1}{p_{m}}\right)\left[\lambda\left(\mathcal{W}_{q, \varepsilon}\right]^{p_{m}}\left|\mathcal{W}_{q, \varepsilon}\right|_{p_{m}}^{p_{m}}\right.
$$


48CAPÍTULO 4. NÚMERO MÍNIMO DE SOLUÇÕES PARA PROBLEMA DE YAMABE-SUBCRÍTICO

além disso pela definição de $\lambda\left(\mathcal{W}_{q, \varepsilon}\right) \mathcal{W}_{q, \varepsilon}$ e pelo limites (4.4),(4.5) e (4.6), temos;

$$
\lambda\left(\mathcal{W}_{q, \varepsilon}\right) \rightarrow 1 \text { quando } \varepsilon \rightarrow 0
$$

decorre que

$$
\lim _{\varepsilon \rightarrow 0} J_{\varepsilon}\left(\lambda\left(\mathcal{W}_{q, \varepsilon}\right) \mathcal{W}_{q, \varepsilon}\right)=E(U)=\mathrm{m}(E)
$$

portanto $\phi_{\varepsilon}(q) \in \sum_{\varepsilon, \mathbf{m}(E)+\delta}$

Considere uma partição finita $\mathcal{P}_{\varepsilon}=\left\{P_{j}^{\varepsilon}\right\}_{j \in \Lambda}$ para $B$. 
Definição 4.15 (Partição boa) Dado um $\varepsilon>0$ dizemos que uma partição $\mathcal{P}_{\varepsilon}=\left\{P_{j}^{\varepsilon}\right\}_{j \in \Lambda}$, com $\Lambda$ finito, de uma variedade $B$ é partição boa se:

1. Para todo $j \in \Lambda$ o conjunto $P_{j}^{\varepsilon}$ é fechado;

2. $P_{j}^{\varepsilon} \cap P_{i}^{\varepsilon} \subseteq \partial P_{j}^{\varepsilon} \cap \partial P_{i}^{\varepsilon}$ para todo $i \neq j$;

3. Existe $\varepsilon>0$ tais que existem pontos $q_{j}^{\varepsilon} \in P_{j}^{\varepsilon}$ para todo $j$, satisfazendo $B\left(q_{j}^{\varepsilon}, \varepsilon\right) \subseteq P_{j}^{\varepsilon} \subseteq$ $B\left(q_{j}^{\varepsilon}, s_{2}(\varepsilon)\right) \subseteq B\left(q_{j}^{\varepsilon}, s_{1}(\varepsilon)\right)$

4. Todo ponto $x \in B$ está contido em no máximo $N$ bolas $B\left(q_{j}^{\varepsilon}, s_{1}(\varepsilon)\right)$, onde $N$ não depende do $\varepsilon$

5. Os números $s_{1}, s_{2}$ podem ser escolhidos de modo que $s_{1} \geq s_{2} \geq(1+1 / K) \varepsilon$, onde $K 4.2 .1$ não depende de $\varepsilon$.

Iremos supor que $\varepsilon_{0}$ do teorema 4.14 é suficientemente pequeno de forma que a variedade $B$ tenha uma partição boa.

Teorema 4.16 Existe uma constante $\gamma>0$ tal que para qualquer $\delta>0$ fixado e qualquer $\varepsilon \in$ $\left(0, \varepsilon_{0}(\delta)\right)$, onde $\varepsilon_{0}(\delta)$ é como em 4.14, considere uma partição boa $\mathcal{P}_{\varepsilon}=\left\{P_{j}^{\varepsilon}\right\}_{j \in \Lambda}$ da variedade $B$ e uma função $u \in \sum_{\varepsilon, m(E)+\delta}$, então existe um conjunto $\tilde{P}_{j}^{\varepsilon} \in \mathcal{P}_{\varepsilon}$ tal que

$$
\frac{1}{\varepsilon^{n}} \int_{\tilde{P}_{j}^{\varepsilon}}\left|u^{+}\right|^{p} d \mu \geq \gamma
$$

\section{Prova:}

Fixado $\delta>0$ e $0<\varepsilon<\varepsilon_{0}(\delta)$. Para qualquer função $u \in N_{\varepsilon}$ e qualquer partição boa $\mathcal{P}_{\varepsilon}=$ $\left\{P_{j}^{\varepsilon}\right\}_{j \in \Lambda}$, denotamos $u_{j}^{+}$como sendo a restrição de $u^{+}$ao conjunto $P_{j}^{\varepsilon}$. Como $u \in N_{\varepsilon}$, decorre que:

$$
\begin{aligned}
\frac{1}{\varepsilon^{n}} \int_{B}\left(\varepsilon^{2}|\nabla u|^{2}+\left(\frac{S_{g_{B}}}{a_{m}} \varepsilon^{2}+1\right) u^{2}\right) d \mu_{g_{B}} & =\frac{1}{\varepsilon^{n}} \int_{B}\left(u^{+}\right)^{p_{m}} d \mu_{g_{B}} \\
& =\frac{1}{\varepsilon^{n}} \sum_{j} \int_{P_{j}^{\varepsilon}}\left(u^{+}\right)^{p_{m}} d \mu_{g_{B}} \\
& =\sum_{j} \frac{\left|u_{j}^{+}\right|_{p_{m}}^{p_{m}-2}}{\varepsilon^{\frac{n\left(p_{m}-2\right)}{p_{m}}}} \cdot \frac{\left|u_{j}^{+}\right|_{p_{m}}^{2}}{\varepsilon^{\frac{2 n}{p_{m}}}} \\
& \leq \max _{j}\left\{\frac{1}{\varepsilon^{\frac{n\left(p_{m}-2\right)}{p_{m}}}} \cdot\left|u_{j}^{+}\right|_{p_{m}}^{p_{m}-2}\right\} \sum_{j} \frac{1}{\varepsilon^{\frac{2 n}{p_{m}}}} \cdot\left|u_{j}^{+}\right|_{p_{m}}^{2}
\end{aligned}
$$

Agora vamos definir uma função cut-off

$$
\chi_{\varepsilon}(t):=\left\{\begin{array}{l}
1 \text { se } t \leq s_{2}(\varepsilon) \\
0 \text { se } t>s_{1}(\varepsilon)
\end{array}\right.
$$

onde $s_{1}(\varepsilon), s_{2}(\varepsilon)$ são definidas junto com a partição boa, vamos supor tambem que $\left|\chi_{\varepsilon}^{\prime}\right| \leq \frac{K}{\varepsilon}$. Defina agora

$$
\tilde{u}_{j}(x)=u^{+}(x) \chi_{\varepsilon}\left(\left|x-q_{j}^{\varepsilon}\right|\right)
$$

onde $\tilde{u}_{j}(x) \in H^{1}(B)$ 
Agora vamos estimar

$$
\begin{array}{rlr}
\frac{1}{\varepsilon^{\frac{2 n}{p_{m}}}} \cdot\left|u_{j}^{+}\right|_{p_{m}}^{2} & \leq \frac{1}{\varepsilon^{\frac{2 n}{p_{m}}}} \cdot\left|\tilde{u}_{j}\right|_{p_{m}}^{2} & \left(P_{j}^{\varepsilon} \subseteq B\left(q_{j}^{\varepsilon}, s_{2}(\varepsilon)\right)\right) \\
& \leq\left. C||\left|\tilde{u}_{j}\right|\right|_{\varepsilon} ^{2} & \text { (usando a desiguldade de Sobolev) } \\
& =C\left|\left\|\tilde { u } _ { j } | _ { P _ { j } ^ { \varepsilon } } \left|\left\|_{\varepsilon}^{2}+C\left|\left\|\left.\tilde{u}_{j}\right|_{B\left(q_{j}^{\varepsilon}, s_{1}(\varepsilon)\right) \backslash P_{j}^{\varepsilon}} \mid\right\|_{\varepsilon}^{2}\right.\right.\right.\right.\right. & \\
& =C\left|\left\|u _ { j } ^ { + } | _ { P _ { j } ^ { \varepsilon } } \left|\left\|_{\varepsilon}^{2}+C\left|\left\|\left.\tilde{u}_{j}\right|_{B\left(q_{j}^{\varepsilon}, s_{1}(\varepsilon)\right) \backslash P_{j}^{\varepsilon}} \mid\right\|_{\varepsilon}^{2}\right.\right.\right.\right.\right. &
\end{array}
$$

onde $C$ é a constante da desigualdade de Sobolev e

$$
\||| u \mid\|_{\varepsilon}^{2}=\frac{1}{\varepsilon^{n}} \int_{B}\left(\varepsilon^{2}|\nabla u|^{2}+\left(\frac{S_{g_{B}}}{a_{m}} \varepsilon^{2}+1\right)|u|^{2}\right) d \mu
$$

Decorre que

$$
\begin{aligned}
& C||\left|\tilde{u}_{j}\right|_{B\left(q_{j}^{\varepsilon}, s_{1}(\varepsilon)\right) \backslash P_{j}^{\varepsilon} \mid}||_{\varepsilon}^{2}= \\
= & C \cdot \frac{1}{\varepsilon^{n}}\left(\int_{B\left(q_{j}^{\varepsilon}, s_{1}(\varepsilon)\right) \backslash P_{j}^{\varepsilon}} \varepsilon^{2}\left|\nabla \tilde{u}_{j}\right|^{2} d \mu+\left(\frac{S_{g_{B}}}{a_{m}} \varepsilon^{2}+1\right) \int_{B\left(q_{j}^{\varepsilon}, s_{1}(\varepsilon)\right) \backslash P_{j}^{\varepsilon}}\left|\tilde{u}_{j}\right|^{2} d \mu\right) \\
\leq & C \cdot \frac{1}{\varepsilon^{n}}\left(\int_{B\left(q_{j}^{\varepsilon}, s_{1}(\varepsilon)\right) \backslash P_{j}^{\varepsilon}}\left(\varepsilon^{2}\left|\nabla u^{+}\right|^{2}+K^{2}\left|u^{+}\right|^{2}\right) d \mu+\left(\frac{S_{g_{B}}}{a_{m}} \varepsilon^{2}+1\right) \int_{B\left(q_{j}^{\varepsilon}, s_{1}(\varepsilon)\right) \backslash P_{j}^{\varepsilon}}\left|u^{+}\right|^{2} d \mu\right) \\
& \text { usamos o fato de que } u_{j} \leq u^{+}
\end{aligned}
$$

Decorre da observação anterior que

$$
\begin{aligned}
& \frac{1}{\varepsilon^{\frac{2 n}{p_{m}}}} \cdot\left|u_{j}^{+}\right|_{p_{m}}^{2} \\
= & C\|\| u_{j}^{+}||_{\varepsilon}^{2}+C \cdot \frac{1}{\varepsilon^{n}}\left(\int_{B\left(q_{j}^{\varepsilon}, s_{1}(\varepsilon)\right) \backslash P_{j}^{\varepsilon}}\left(\varepsilon^{2}\left|\nabla u^{+}\right|^{2}+K^{2}\left|u^{+}\right|^{2}\right) d \mu+\left(\frac{S_{g_{B}}}{a_{m}} \varepsilon^{2}+1\right) \int_{B\left(q_{j}^{\varepsilon}, s_{1}(\varepsilon)\right) \backslash P_{j}^{\varepsilon}}\left|u^{+}\right|^{2} d \mu\right) \\
= & C\left\||| u_{j}^{+}|\||_{\varepsilon}^{2}+C \cdot \frac{1}{\varepsilon^{n}}\left(\int_{B\left(q_{j}^{\varepsilon}, s_{1}(\varepsilon)\right) \backslash P_{j}^{\varepsilon}}\left(\varepsilon^{2}\left|\nabla u^{+}\right|^{2}+\left(K^{2}+1\right)\left(\frac{S_{g_{B}}}{a_{m}} \varepsilon^{2}+1\right)\left|u^{+}\right|^{2}\right) d \mu\right)\right. \\
= & \left.C\left\||| u_{j}^{+}\right\|\right|_{\varepsilon} ^{2}+\left.C\left(K^{2}+1\right)\left|\| u^{+}\right|\right|_{\varepsilon} ^{2}
\end{aligned}
$$

Aplicando o samatório em $j$, temos

$$
\begin{aligned}
\sum_{j} \frac{1}{\varepsilon^{\frac{2 n}{p_{m}}}} \cdot\left|u_{j}^{+}\right|_{p_{m}}^{2} & \leq \sum_{j} C\left|\left\|u _ { j } ^ { + } \left|\left\|_{\varepsilon}^{2}+\left.C\left(K^{2}+1\right) N\left|\| u^{+}\right|\right|_{\varepsilon} ^{2}\right.\right.\right.\right. \\
& \leq C\left(K^{2}+2\right) N \cdot \frac{1}{\varepsilon^{n}} \int_{B}\left(\varepsilon^{2}|\nabla u|^{2}+\left(\frac{S_{g_{B}}}{a_{m}} \varepsilon^{2}+1\right)|u|^{2}\right) d \mu
\end{aligned}
$$


Portanto

$$
\begin{aligned}
& \frac{1}{\varepsilon^{n}} \int_{B}\left(\varepsilon^{2}|\nabla u|^{2}+\left(\frac{S_{g_{B}}}{a_{m}} \varepsilon^{2}+1\right) u^{2}\right) d \mu \leq \\
& \leq \max _{j}\left\{\frac{1}{\varepsilon^{\frac{n\left(p_{m}-2\right)}{p_{m}}}} \cdot\left|u_{j}^{+}\right|_{p_{m}}^{p_{m}-2}\right\} \sum_{j} \frac{1}{\varepsilon^{\frac{2 n}{p_{m}}}} \cdot\left|u_{j}^{+}\right|_{p_{m}}^{2} \\
& \leq \max _{j}\left\{\frac{1}{\varepsilon^{\frac{n\left(p_{m}-2\right)}{p_{m}}}}\right\} \cdot C\left(K^{2}+2\right) N \cdot \frac{1}{\varepsilon^{n}} \int_{B}\left(\varepsilon^{2}|\nabla u|^{2}+\left(\frac{S_{g_{B}}}{a_{m}} \varepsilon^{2}+1\right)|u|^{2}\right) d \mu
\end{aligned}
$$

Podemos concluir que

$$
\max _{j}\left\{\left(\frac{1}{\varepsilon^{n}} \int_{p_{j}^{\varepsilon}}\left|u^{+}\right|_{m}^{p}\right)^{\frac{p_{m}-2}{p_{m}}}\right\} \geq \overbrace{\frac{1}{C\left(K^{2}+2\right) N}}^{\gamma}
$$

Teorema 4.17 Fixe $r<r_{0}$, para $\eta<1$ existem $\varepsilon_{0}>0$ e $\delta_{0}>0$ tais que para $\varepsilon \in\left(0, \varepsilon_{0}\right), \delta \in\left(0, \delta_{0}\right)$ e $u \in \sum_{\varepsilon, m_{\varepsilon}+\delta}$, existe $x \in B$ tal que

$$
\int_{B(x, r)}\left(u^{+}\right)^{p} \geq \eta \int_{B}\left(u^{+}\right)^{p}
$$

\section{Prova:}

Suponhamos que o teorema seja falso, portanto existem $\eta<1$ e uma sequencia de números positivos $\left(\varepsilon_{k}\right)$ e $\left(\delta_{k}\right)$ tal que $\varepsilon_{k} \longrightarrow 0$ e $\delta_{k} \longrightarrow 0$ e $u_{k} \in \sum_{\varepsilon_{k}, m_{\varepsilon}+\delta_{k}}$ de forma que

$$
\int_{B(x, r)}\left(u^{+}\right)^{p}<\int_{B}\left(u_{k}^{+}\right)^{p}
$$

para $k$ suficientemente grande, existe $x_{k} \in B$ para $\gamma>0$ fixado, decorre que

$$
\frac{1}{\varepsilon_{k}^{n}} \int_{B\left(x_{k}, 2 \varepsilon_{k}\right)}\left(u_{k}^{+}\right) \geq \gamma
$$

o lema 3.4 de [16] fornece uma função $\tilde{u}_{k}=u_{k_{j}}+u_{k_{i}}$ tal que $\tilde{u}_{k} \in \sum_{\varepsilon_{k}, m_{\varepsilon}+2 \delta_{k}}$, onde $u_{k_{j}}$ tem suporte em uma bola centrada em $x_{k}$ e $\operatorname{supp} u_{k_{j}} \cap \operatorname{supp} u_{k_{i}}=\varnothing$, com $u_{j}=\tilde{u}_{k}$ em $B_{\varepsilon_{k}}\left(x_{k}\right)$ e fora de $B_{r}\left(x_{k}\right)$.

$$
\frac{1}{\varepsilon_{k}^{n}} \int_{B}\left(u_{k_{j}}^{+}\right)^{p} \geq \gamma
$$

além disso

$$
\frac{1}{\varepsilon_{k}^{n}} \int_{B}\left(u_{k_{i}}^{+}\right)^{p} \geq \frac{1}{\varepsilon_{k}^{n}}(1-\eta) \geq \int_{B}\left(u_{k}^{+}\right)^{p} \geq(1-\eta) \frac{2 p}{p-2} \mathrm{~m}_{\varepsilon}
$$

Pelo corolário 3.3 de [16] exists $\delta_{1}>0$ que não depende de $k$ e uma constante $\rho$ que depende de $\delta_{1}$ portando $J_{\varepsilon_{k}}\left(\tilde{u}_{k}\right) \geq \rho\left(\delta_{1}\right) \cdot \mathrm{m}_{\varepsilon}$, contudo para um $k$ arbitrariamente grande teremos $J_{\varepsilon_{k}}\left(\tilde{u}_{k}\right)<$ 
$\mathrm{m}_{\varepsilon}+2 \delta<\rho\left(\delta_{1}\right) \mathrm{m}_{\varepsilon}$. Contradição e portanto o teorema é verdadeiro.

\subsubsection{Demonstração do Teorema Principal}

\section{Prova:}

Demonstração do Resultado Principal-Teorema 3.1 Fixados os valores $r<(1 / 2) r_{0}$ e $\eta$ proximo de 1 , considere $\varepsilon_{0}, \delta_{0}$ como nas hipóteses dos Teorema 4.17. Teorema 4.14 garante a continuidade da função $\phi_{\varepsilon}: B \rightarrow N_{\varepsilon}$. Para $\varepsilon<\varepsilon_{0}$ o Teorema 4.17 mostra que se $u \in \sum_{\varepsilon, \mathbf{m}_{\varepsilon}+\delta}$ teremos que $\left(u^{+}\right)^{p} \in L_{r, \eta}^{1}(B)$, Agora observe a função contínua $\psi_{\varepsilon}:=c_{m}(r, \eta): L_{r, \eta}^{1}(B) \rightarrow B$, por construção o suporte de $p h i_{\varepsilon}$ esta contido na bola geodésica $B(x, 2 r)$, para $2 r<r_{0}$, dessa forma teremos que

$$
\psi_{\varepsilon} \circ \phi_{\varepsilon}(x) \in B(x, 2 r)
$$

além disso podemos definir uma homotopia entre $\psi_{\varepsilon} \circ \phi_{\varepsilon}: B \longrightarrow B$ e $I d_{B}$, pela unicidade da geodesica minimizante que liga $\left(\psi_{\varepsilon} \circ \phi_{\varepsilon}\right)(x)$ a $x$. Pelo Teorema 4.9 teremos $\operatorname{cat}(B) \leq \operatorname{cat}\left(\sum_{\left.\varepsilon, \mathbf{m}_{\varepsilon}+\delta\right)}\right.$ aplicando o teorema 4.8 , podemos concluir que $J_{\varepsilon}$ tem pelo menos cat $(B)+1$ pontos críticos. 


\section{Referências Bibliográficas}

[1] Kazuo Akutagawa, Luis A Florit, and Jimmy Petean. On yamabe constants of riemannian products. arXiv preprint math/0603486, 2006.

[2] Thierry Aubin et al. Métriques riemanniennes et courbure. Journal of Differential Geometry, 4(4):383-424, 1970.

[3] Vieri Benci, Claudio Bonanno, and Anna Maria Micheletti. On the multiplicity of solutions of a nonlinear elliptic problem on riemannian manifolds. Journal of Functional Analysis, 252(2):464-489, 2007.

[4] Vieri Benci and Giovanna Cerami. The effect of the domain topology on the number of positive solutions of nonlinear elliptic problems. Archive for Rational Mechanics and Analysis, 114(1):79-93, 1991.

[5] Renato Bettiol and Paolo Piccione. Multiplicity of solutions to the yamabe problem on collapsing riemannian submersions. Pacific Journal of Mathematics, 266(1):1-21, 2013.

[6] Renato G Bettiol and Paolo Piccione. Bifurcation and local rigidity of homogeneous solutions to the yamabe problem on spheres. Calculus of Variations and Partial Differential Equations, 47(3-4):789-807, 2013.

[7] Renato G Bettiol, Paolo Piccione, Bianca Santoro, et al. Bifurcation of periodic solutions to the singular yamabe problem on spheres. Journal of Differential Geometry, 103(2):191-205, 2016 .

[8] LL De Lima, Paolo Piccione, and Michela Zedda. On bifurcation of solutions of the yamabe problem in product manifolds. In Annales de l'Institut Henri Poincare (C) Non Linear Analysis, volume 29, pages 261-277. Elsevier, 2012.

[9] M.P. do Carmo. Geometria riemanniana. Projeto Euclides. Instituto de Matemática Pura e Aplicada, 1988.

[10] Basilis Gidas, Wei-Ming Ni, and Louis Nirenberg. Symmetry and related properties via the maximum principle. Communications in Mathematical Physics, 68(3):209-243, 1979.

[11] Karsten Grove and Hermann Karcher. How to conjugatec 1-close group actions. Mathematische Zeitschrift, 132(1):11-20, 1973.

[12] David Hilbert. Die grundlagen der physik. Mathematische Annalen, 92(1):461-472, 1924.

[13] Hermann Karcher. Riemannian center of mass and mollifier smoothing. Communications on pure and applied mathematics, 30(5):509-541, 1977. 
[14] Ana Claudia da Silva Moreira. Técnicas de bifurcação para o problema de Yamabe em variedades com bordo. PhD thesis, Universidade de São Paulo.

[15] Morio Obata. The conjectures on conformal transformations of riemannian manifolds. Bulletin of the American Mathematical Society, 77(2):265-270, 1971.

[16] Jimmy Petean. Multiplicity results for the yamabe equation by lusternik-schnirelmann theory. arXiv preprint arXiv:1611.01177, 2016.

[17] Daniel Pollack. Nonuniqueness and high energy solutions for a conformally invariant scalar equation. Comm. Anal. Geom, 1(3-4):347-414, 1993.

[18] Richard Schoen et al. Conformal deformation of a riemannian metric to constant scalar curvature. Journal of Differential Geometry, 20(2):479-495, 1984.

[19] Gaetano Siciliano. Multiple positive solutions for a schrödinger-poisson-slater system. Journal of mathematical analysis and applications, 365(1):288-299, 2010.

[20] Neil S Trudinger. Remarks concerning the conformal deformation of riemannian structures on compact manifolds. Annali della Scuola Normale Superiore di Pisa-Classe di Scienze, $22(2): 265-274,1968$.

[21] Hidehiko Yamabe. On a deformation of riemannian structures on compact manifolds. 1960. 